\title{
ANALYSIS OF DOSIMETRIC DIFFERENCES BETWEEN DOSE-TO-WATER AND DOSE-TO-MEDIUM CALCULATIONS FOR ELECTRON BEAMS
}

\author{
by \\ Elena Gil \\ B.Sc. in Physics (2007) \\ A thesis submitted to the \\ Faculty of Graduate Studies and Research \\ in partial fulfillment of the requirements \\ for the degree of \\ Master of Science \\ Ottawa-Carleton Institute for Physics \\ Department of Physics, Carleton University \\ Ottawa, Ontario, Canada \\ December 2009 \\ (C) Copyright 2009, Elena Gil
}


Library and Archives

Canada

Published Heritage

Branch

395 Wellington Street Ottawa ON K1A ON4 Canada
Bibliothèque et

Archives Canada

Direction du

Patrimoine de l'édition

395, rue Wellington Ottawa ON K1A ON4

Canada
Your file Votre référence

ISBN: 978-0-494-64436-2

Our file Notre réference

ISBN: 978-0-494-64436-2
NOTICE:

The author has granted a nonexclusive license allowing Library and Archives Canada to reproduce, publish, archive, preserve, conserve, communicate to the public by telecommunication or on the Internet, loan, distribute and sell theses worldwide, for commercial or noncommercial purposes, in microform, paper, electronic and/or any other formats.

The author retains copyright ownership and moral rights in this thesis. Neither the thesis nor substantial extracts from it may be printed or otherwise reproduced without the author's permission.
AVIS:

L'auteur a accordé une licence non exclusive permettant à la Bibliothèque et Archives Canada de reproduire, publier, archiver, sauvegarder, conserver, transmettre au public par télécommunication ou par l'Internet, prêter, distribuer et vendre des thèses partout dans le monde, à des fins commerciales ou autres, sur support microforme, papier, électronique et/ou autres formats.

L'auteur conserve la propriété du droit d'auteur et des droits moraux qui protège cette thèse. $\mathrm{Ni}$ la thèse ni des extraits substantiels de celle-ci ne doivent être imprimés ou autrement reproduits sans son autorisation.
In compliance with the Canadian Privacy Act some supporting forms may have been removed from this thesis.

While these forms may be included in the document page count, their removal does not represent any loss of content from the thesis.
Conformément à la loi canadienne sur la protection de la vie privée, quelques formulaires secondaires ont été enlevés de cette thèse.

Bien que ces formulaires aient inclus dans la pagination, il n'y aura aucun contenu manquant. 


\begin{abstract}
In cancer radiation therapy, the conventional approach has been to calculate and report dose in terms of dose-to-water. With the introduction of commercial Monte Carlo based treatment planning systems, it is now possible to calculate and evaluate dose distributions in terms of dose-to-medium, as opposed to the traditional dose-to-water, of conventional planning systems. The two calculation approaches are conceptually different and the method used has an impact on the reported doses to different organs, such as bone and lung. For electron beams, differences between the two methods have been reported to exceed $10 \%$ in cases with hard bone. These differences are greatest in materials whose electron densities are furthest from water, such as bone and lung. This has raised the question of which approach is more appropriate. An AAPM task group has recently recommended that both options should be available in commercial software, but as of yet, there is no consensus which approach should be used. There is a need for systematic analysis of clinical data that would help with the understanding of differences between the $D_{m}$ and $D_{w}$ approaches, which in turn could lead to a more accurate evaluation of treatment outcomes. The purpose of this study is to investigate the dosimetric differences between plans calculated using the $D_{m}$ versus $D_{w}$ approach for clinical breast, and head and neck cases treated with electron beams. The analysis included plans for epoxy-resin-
\end{abstract}


based phantoms containing hard bone, lung, and air heterogeneities, as well as a retrospective study breast, and head and neck cancer patients treated with electron beams. In some cases, where hard bone was present, differences between $D_{m}$ and $D_{w}$ exceeded $10 \%$. Differences were consistent with the water-to-tissue stopping power. 


\section{Acknowledgements}

I would like to gratefully acknowledge my supervisor, Joanna Cygler. Her guidance and teaching has been invaluable.

My deepest thanks go to Petrusia, Shubi, Bianca, Anita, Dave, Gemma, and Joel. Due to their patience, and understanding, our friendships have managed to survive both distance and time.

Moving to Ottawa, I never expected to meet and befriend such wonderful people. I am so grateful to all the people in the Carleton Physics Community. Lindsay, Amanda, Maria, Dan, Liz, Amir, Jared, Tara and so many others have helped make my time at Carleton entertaining and stimulating. I look forward to many more laughs and adventures.

Ken and Sunoj are not in physics, but they are among the kindest and funniest people I know, and I truly appreciate the friendship we have.

It is impossible to overstate my gratitude to my parents, brothers, and sister-in-laws. They have been a source of strength, providing me with encouragement, guidance, and love. There are no words to express how thankful for all that they have done for me. especially my parents.

Lastly, and most importantly, I wish to thank my husband Peter. His sense of humor and patience has been limitless, and for this I owe him my sanity. I could not have asked for a better husband or friend. I love you. 


\section{Contents}

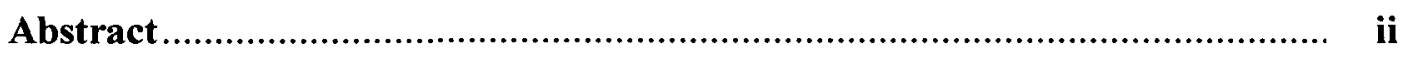

List of Tables

List of Figures ..................................................................................... viii

List of Abbreviations

1. Introduction ...................................................................................... 1

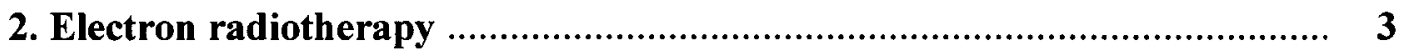

2.1 Characterizing electron dose profiles........................................................ 3

2.2 Interactions and energy loss ............................................................... $\quad 7$

2.2.1 Stopping powers ...................................................................... 7

2.2.2 Scattering powers........................................................................ 10

2.2.3 Dose: The absorption of Energy ..................................................... 11

3. Theraplan Plus ${ }^{\circledR}$ : A Monte Carlo based treatment planning system .......... 13

3.1 Anatomy modeling ............................................................................ 13

3.2 Creating a treatment plan and viewing dose calculations ........................... 14

3.3 DCM: Dose calculation module ......................................................... 15

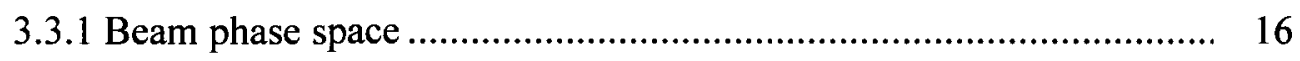

3.3.2 In-patient dose calculations ........................................................ 17

4. Dose-to-water vs. dose-to-medium ……………….................................... 22

5. Proof-of-concept phantoms ................................................................... 26

5.1 Proof-of-concept phantom configurations ………...................................... 26

5.2 Calculations for proof-of-concept phantoms ………….............................. 28

5.2.1 Results: Lung slab phantom ....................................................... 28

5.2.2 Results: Soft bone slab phantom ................................................... 29 
5.2.3 Results: Hard bone slab phantom ................................................... 31

6. Epoxy resin based phantoms ……....................................................... 33

6.1 Epoxy resin based phantom configurations.............................................. 33

6.2 Calculation of treatment plans for epoxy resin vector-based phantoms ........ 38

6.2.1 Results: Hard bone slab epoxy resin vector-based phantom ............... 40

6.2.2 Results: Lung slab epoxy resin vector-based phantom....................... 43

6.2.3 Results: Hard bone cylinder epoxy resin vector-based phantom ........ 46

6.2.4 Results: Air cylinder epoxy resin vector-based phantom ................... 51

6.2.5 Results: Trachea and spine epoxy resin vector-based phantom .......... 54

6.3 Calculation of treatment plans for epoxy resin image-based phantoms ........ 58

6.3.1 Results: Hard bone slab epoxy resin image-based phantom ............... 59

6.3.2 Results: Lung slab epoxy resin image-based phantom ........................ 62

6.3.3 Results: Hard bone cylinder epoxy resin image-based phantom......... 66

6.3.4 Results: Air cylinder epoxy resin image-based phantom................... $\quad 70$

6.3.5 Results: Trachea and spine epoxy resin image-based phantom .......... 73

6.4 Measurement of dose in the epoxy resin based phantoms using film............ 76

6.4.1 Calibration of GafChromic Film ................................................... 76

6.4.2 Film setup within the epoxy resin based phantoms ............................ 78

6.4.3 Results: Film measurement results ................................................... 81

7. Retrospective Patient Data ..................................................................... 86

7.1 Breast cancer patients............................................................................ 86

7.2 Head and neck cancer patients.............................................................. 89

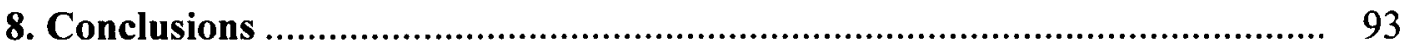

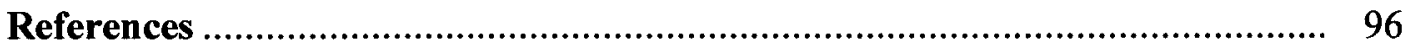




\section{List of Tables}

Table 1 Relative electron densities used for proof-of-concept mathematical phantoms as seen in preliminary patient plans

Table 2 Physical and relative electron densities of the epoxy resin based tissue substitute materials, adapted from White et al.....

Table 3 Treatment plan parameters used to create the vector and image based treatment plans for the epoxy resin phantoms.

Table 4 Image-based and vector-based anatomy properties. Variation in voxel size is due to variations in the size of the external dimensions of the phantom.

Table 5 The mean and range of $D_{w}-$ to- $D_{m}$ ratios in DVH for the breast patients analyzed. 


\section{List of Figures}

Figure 1 Normalized depth dose profiles of a $10 \times 10 \mathrm{~cm}^{2}$ beam at $100 \mathrm{~cm}$ SSD for electron beams of various energies.

Figure 2 Collisional mass stopping powers of water, hard bone, soft bone, soft tissue, air and lung. Inset is a zoomed in view of the range of energies, 1-25 MeV. Values obtained using NIST ESTAR ${ }^{18}$.

Figure 3 Presence of hot and cold spots can be visible in the presence of small inhomogeneities such as hard bone in water. This drawing was adapted from $\mathrm{Khan}^{20}$.

Figure 4 The user defined display matrix (shown in green) does not necessarily align with the calculation matrix (shown in black) defined by the algorithm.

Figure 5 Voxels in the calculation matrix are assigned material properties based on the average HU numbers within the voxel. The larger grid represents the pixels of the calculation matrix, the smaller grid represents the pixels of the phantom/patient image. For clarity, the display matrix is not shown. The image to the left is an example of an inhomogeneity with the calculation matrix superimposed on it prior to averaging the $\mathrm{HU}$ values of the image pixels. In the image to the right, the voxels that are fully shaded have been assigned the proper material properties of the inhomogeneity.

Figure 6 Workflow of the Monte Carlo simulation for each particle.

Figure 7 Ratio of unrestricted mass collisional stopping power ratios of water to material. Values were obtained from NIST ESTAR ${ }^{18}$ for monoenergetic electrons of energies 0.01 to $1000 \mathrm{MeV}$. Inset is of a zoomed view of the range of energies. 
Figure 8 Structural depiction of the proof of concept mathematical phantoms. The external anatomy is outlined in black, while the contoured inhomogeneity is shown in solid blue. The green cube delineates the external (electron) beam. The central transverse slice is shown to the right.

Figure 9 Axial dose profiles along the central axis of the proof-of-concept lung phantom irradiated with a (a) $9 \mathrm{MeV}$ beam, (b) $17 \mathrm{MeV}$ beam. Profiles were normalized to $\mathrm{D}_{\max }$ in a homogenous water phantom.

Figure 10 Axial dose profiles along the central axis of the proof-of-concept soft bone phantom irradiated with a (a) $9 \mathrm{MeV}$ beam, (b) $17 \mathrm{MeV}$ beam. Profiles were normalized to $D_{\max }$ in a homogenous water phantom.

Figure 11 Axial dose profiles along the central axis of the proof-of-concept hard bone phantom irradiated with a (a) $9 \mathrm{MeV}$ beam, (b) $17 \mathrm{MeV}$ beam. Profiles were normalized to $\mathrm{D}_{\max }$ in a homogenous water phantom.

Figure 12 The different configurations of the epoxy resin based phantoms used for $D_{m}$ and $D_{w}$ calculations.

Figure 13 Axial and lateral dose profiles, DVH and dose difference map for the vector-based hard bone slab phantom, calculated to deliver $200 \mathrm{MU}$ with a $9 \mathrm{MeV}$ beam. Profiles were normalized to $D_{\max }$ in a homogenous water phantom.

Figure 14 Axial and lateral dose profiles, DVH and dose difference map for the vector-based hard bone slab phantom, calculated to deliver $200 \mathrm{MU}$ with a $17 \mathrm{MeV}$ beam. Profiles were normalized to $D_{\max }$ in a homogenous water phantom.

Figure 15 Axial and lateral dose profiles, DVH and dose difference map for the vector-based lung slab phantom, calculated to deliver $200 \mathrm{MU}$ with a $9 \mathrm{MeV}$ beam. Profiles were normalized to $D_{\max }$ in a homogenous water phantom.

Figure 16 Axial and lateral dose profiles, DVH and dose difference map for the vector-based lung slab phantom, calculated to deliver $200 \mathrm{MU}$ with a $17 \mathrm{MeV}$ beam. Profiles were normalized to $D_{\max }$ in a homogenous water phantom.

Figure 17 Axial and lateral dose profiles, DVH and dose difference map for the vector-based hard bone cylinder phantom (bone $1.2 \mathrm{~cm}$ from the surface), calculated to deliver $200 \mathrm{MU}$ with a $9 \mathrm{MeV}$ beam. Profiles were normalized to $\mathrm{D}_{\max }$ in a homogenous water phantom. 
Figure 18 Display matrix (green grid) superimposed on the calculation matrix (black grid) and the hard bone cylinder phantom.

Figure 19 Axial and lateral dose profiles, DVH and dose difference map for the vector-based hard bone cylinder phantom (bone $1.2 \mathrm{~cm}$ from the surface), calculated to deliver $200 \mathrm{MU}$ with a $17 \mathrm{MeV}$ beam. Profiles were normalized to $\mathrm{D}_{\max }$ in a homogenous water phantom.

Figure 20 Axial and lateral dose profiles, DVH and dose difference map for the vector-based air cylinder phantom (cylinder $1.2 \mathrm{~cm}$ from the surface), calculated to deliver $200 \mathrm{MU}$ with a $9 \mathrm{MeV}$ beam. Profiles were normalized to $D_{\max }$ in a homogenous water phantom.

Figure 21 Axial and lateral dose profiles, DVH and dose difference map for the vector-based air cylinder phantom (cylinder $1.2 \mathrm{~cm}$ from the surface), calculated to deliver $200 \mathrm{MU}$ with a $17 \mathrm{MeV}$ beam. Profiles were normalized to $\mathrm{D}_{\max }$ in a homogenous water phantom.

Figure 22 Display matrix (green grid) superimposed on the calculation matrix (black grid) and the air cylinder phantom.

Figure 23 Axial and lateral dose profiles, DVH and dose difference map for the vector-based trachea and spine phantom, calculated to deliver $200 \mathrm{MU}$ with a $9 \mathrm{MeV}$ beam. The lateral dose profile contains a pictorial description of where the profile was taken within the phantom. Profiles were normalized to $\mathrm{D}_{\max }$ in a homogenous water phantom.

Figure 24 Axial and lateral dose profiles, DVH and dose difference map for the vector-based trachea and spine phantom, calculated to deliver $200 \mathrm{MU}$ with a $17 \mathrm{MeV}$ beam. The lateral dose profile contains a pictorial description of where the profile was taken within the phantom. Profiles were normalized to $D_{\max }$ in a homogenous water phantom.

Figure 25 Depth of lateral profiles with respect to the calculation matrix (black grid), display matrix (green matrix), and the inhomogeneities. The 9 and $17 \mathrm{MeV}$ lateral profiles are shown in red and orange respectively.

Figure 26 Axial and lateral dose profiles, DVH and dose difference map for the image-based hard bone slab phantom, calculated to deliver $200 \mathrm{MU}$ with a $9 \mathrm{MeV}$ beam. Profiles were normalized to $D_{\max }$ in a homogenous water phantom.

Figure 27 Axial and lateral dose profiles, DVH and dose difference map for the image-based hard bone slab phantom, calculated to deliver $200 \mathrm{MU}$ with a $17 \mathrm{MeV}$ beam. Profiles were normalized to $D_{\max }$ in a homogenous water phantom. 
Figure 28 Axial and lateral dose profiles, DVH and dose difference map for the image-based lung slab phantom, calculated to deliver $200 \mathrm{MU}$ with a $9 \mathrm{MeV}$ beam. Profiles were normalized to $D_{\max }$ in a homogenous water phantom.

Figure 29 Axial and lateral dose profiles, DVH and dose difference map for the image-based lung slab phantom, calculated to deliver $200 \mathrm{MU}$ with a $17 \mathrm{MeV}$ beam. Profiles were normalized to $D_{\max }$ in a homogenous water phantom.

Figure 30 Axial dose profiles of the vector-based and image-based lung slab phantom for (a) $9 \mathrm{MeV}$ and (b) $17 \mathrm{MeV}$. Profiles were normalized to $\mathrm{D}_{\max }$ in a homogenous water phantom.

Figure 31 Axial and lateral dose profiles, DVH and dose difference map for the image-based hard bone cylinder phantom, calculated to deliver 200 $\mathrm{MU}$ with a $9 \mathrm{MeV}$ beam. Profiles were normalized to $\mathrm{D}_{\max }$ in a homogenous water phantom.

Figure 32 Axial and lateral dose profiles, DVH and dose difference map for the image-based hard bone cylinder phantom, calculated to deliver 200 MU with a $17 \mathrm{MeV}$ beam. Profiles were normalized to $D_{\max }$ in a homogenous water phantom.

Figure 33 Display matrix superimposed on the calculation matrix and the imagebased bone cylinder phantom.

Figure 34 Axial dose profiles of the vector-based and image-based hard bone cylinder phantom for (a) $9 \mathrm{MeV}$ and (b) $17 \mathrm{MeV}$. Profiles were normalized to $\mathrm{D}_{\max }$ in a homogenous water phantom.

Figure 35 Axial and lateral dose profiles, DVH and dose difference map for the image-based air cylinder phantom, calculated to deliver $200 \mathrm{MU}$ with a $9 \mathrm{MeV}$ beam. Profiles were normalized to $D_{\max }$ in a homogenous water phantom.

Figure 36 Axial and lateral dose profiles, DVH and dose difference map for the image-based air cylinder phantom, calculated to deliver $200 \mathrm{MU}$ with a $17 \mathrm{MeV}$ beam. Profiles were normalized to $D_{\max }$ in a homogenous water phantom.

Figure 37 Axial and lateral dose profiles, DVH and dose difference map for the image-based trachea \& spine phantom, calculated to deliver $200 \mathrm{MU}$ with a $9 \mathrm{MeV}$ beam. The lateral dose profile contains a pictorial description of where the profile was taken within the phantom. Profiles were normalized to $D_{\max }$ in a homogenous water phantom. 
Figure 38 Axial and lateral dose profiles, DVH and dose difference map for the image-based trachea \& spine phantom, calculated to deliver $200 \mathrm{MU}$ with a $17 \mathrm{MeV}$ beam. The lateral dose profile contains a pictorial description of where the profile was taken within the phantom. Profiles were normalized to $D_{\max }$ in a homogenous water phantom.

Figure $399 \mathrm{MeV}$ and $17 \mathrm{MeV}$ GafChromic EBT1 film calibration curve.

Figure 40 Location of GafChromic EBT1 film within slab geometry phantoms. .... 79

Figure 41 Location of the GafChromic EBT1 film within the hard bone and air cylinder phantoms.

Figure 42 Location of the GafChromic EBT1 film within the trachea and spine phantom.

Figure $439 \mathrm{MeV}$ film measurements and calculations at various depths within the hard bone slab phantom. To the left are the film measurements and vector based calculations, to the right are the film measurements and the image based calculations.

Figure $44 \quad 17 \mathrm{MeV}$ film measurements and calculations at various depths within the hard bone slab phantom. To the left are the film measurements and vector based calculations, to the right are the film measurements and the image based calculations.

Figure $459 \mathrm{MeV}$ film measurements and calculations at various depths within the lung slab phantom. To the left are the film measurements and vector based calculations, to the right are the film measurements and the image based calculations.

Figure $46 \quad 17 \mathrm{MeV}$ film measurements and calculations at various depths within the lung slab phantom. To the left are the film measurements and vector based calculations, to the right are the film measurements and the image based calculations.

Figure 47 DVH comparison of $D_{w}$ and $D_{m}$ for a right breast mastectomy patient treated with a $13 \mathrm{MeV}$ electron beam, at $\mathrm{SSD}=115 \mathrm{~cm}$, illustrating subtle dose differences in (a) the sternum and (b) the internal mammary nodes.

Figure 48 Isodose line distributions, calculated for $D_{w}$ (a) and $D_{m}$ (b) for a patient treated with $13 \mathrm{MeV}$ electrons. There is little visual difference between these plans. Blue dashed lines on (a) and (b) indicate the depth at which the lateral dose profiles in (c) were taken. 
Figure $49 \quad D_{m}$ and $D_{w}$ profiles of a patient treated with a $6 \mathrm{MeV}$ beam positioned perpendicular to the patient's forehead on the right side. (a) $\mathrm{D}_{\mathrm{m}}$ isodose distribution. (b) $D_{w}$ isodose distribution. (c) $D_{m}$ and $D_{w}$ profiles through the forehead, denoted by the dotted lines in (a) and (b), respectively. There is a maximum difference of $7.9 \%$ between the $D_{m}$ and $D_{w}$ profiles, where $D_{w}$ exceeds $D_{m}$ 


\title{
List of Abbreviations
}

\author{
CT ................ Computed tomography \\ CTV ............. Clinical target volume \\ $\mathrm{cm}$................ Centimeters, unit of length \\ DCM ............. Dose calculation module \\ $\mathrm{D}_{\mathrm{m}}$................ Dose-to-medium \\ $\mathrm{D}_{\max }$............... Maximum dose \\ $D_{w}$................ Dose-to-water \\ $\mathrm{d}_{\max }$............... Depth of maximum dose \\ DVH............. Dose volume histogram \\ ERB ............ Epoxy resin based \\ g ................. Grams, units of mass \\ HU ............... Housnfield unit \\ GTV ............. Gross target volume \\ $\mathrm{L} / \rho$............... Restricted mass stopping power \\ Linac ............. Linear accelerator \\ MC ................ Monte Carlo \\ $\mathrm{MeV}$.............. Mega electron volts, unit of energy \\ MU................ Monitor units \\ OAR ............. Organ at risk \\ PDD ............. Percent depth dose \\ PTV .............. Planning target volume \\ RED ............. Relative electron density \\ S/p............... Unrestricted mass stopping power \\ SPR ............. Stopping power ratio \\ SSD .............. Source-to-surface distance \\ TPS ............. Treatment planning system
}




\section{Chapter 1}

\section{Introduction}

Radiation therapy is the use of ionizing radiation to damage cancerous tissue within the body, and has been a form of treatment since the early 1900 's ${ }^{1}$. The continued development of this form of therapy saw the introduction of high-energy electrons as a form of cancer treatment in the early 1950 's ${ }^{2}$, as well as other significant advancements in both treatment and treatment planning. One of the recent advancements has been the introduction of Monte Carlo (MC) based treatment planning systems (TPS), a highly accurate method of calculating dose distributions within a medium.

Traditionally in cancer radiation therapy, dose has been calculated and reported in terms of dose-to-water ${ }^{3}, D_{w}$. This can partially be attributed to the $D_{w}$-based calibration protocols used in the clinic for the calibration of instrumentation ${ }^{4,5}$ and the inability of early dose calculation algorithms to account for inhomogeneities. However, the human body is made up of a variety of materials, including bone and lung. Monte Carlo based treatment planning systems in clinics have made it possible to calculate and report plans in terms of dose-to-medium, $\mathrm{D}_{\mathrm{m}}$. The two methods are fundamentally different ${ }^{6,7}$, and yet no standard method of calculating or reporting dose exists ${ }^{8}$. An AAPM task group ${ }^{9}$ has recently recommended that both options be available in commercial software, since

the method used has an impact on the reported dose ${ }^{10}$, and therefore on the evaluation of treatment outcomes.

There is a need for a systematic analysis of clinical data to understand the differences between the $D_{m}$ and $D_{w}$ approaches which could in turn lead to a more accurate evaluation of treatment outcomes. The purpose of this study was to analyze possible 
differences that may exist between electron beam therapy plans calculated using the $D_{m}$ and $D_{w}$ approaches. These differences were also investigated in clinical breast, and head and neck cancer treatment plans. 


\section{Chapter 2}

\section{Electron radiotherapy}

The types of radiation typically used in clinics are photon and electron beams, but other types of ionizing radiation, such as protons, are gaining popularity ${ }^{11}$. The type of radiation chosen to treat a patient is selected based on a variety of factors including the dose distribution characteristics of the radiation, as well as the location and size of the lesion $^{12}$. Electron beam therapy is used mainly for the treatment of superficial and moderately deep tumors ${ }^{12}$, typically less than $6 \mathrm{~cm}$ deep. Electron beams are preferred for these tumors since they deposit most of their energy near the surface and have a characteristically sharp energy drop off beyond the depth of maximum dose, $d_{\max }$. This is useful in protecting any healthy tissue behind the tumor from receiving unnecessary dose, while being able to deliver the prescribed dose to the lesion. Clinically, the range of energy used for treatment is $4-25 \mathrm{MeV}$. The energy used to treat a patient is selected based on the depth of the tumor, the prescribed dose to the tumor and the dose to the critical organs ${ }^{12}$. The goal to is to deliver the prescribed dose to the lesion while sparing the healthy tissue around it. This thesis focuses on external electron beam treatment plans, specifically in the case of breast, and head and neck patients.

\subsection{Characterizing electron dose profiles}

The dose distribution in a medium depends on the energy of the incident beam. This distribution can be characterized by examining the dose deposition through a medium along the central axis of the beam, also known as the depth-dose profile or axial dose profile. If the dose profile is normalized to the maximum dose, the axial profile is 
referred to as a percent depth dose (PDD) profile. Figure 1 shows PDD profiles in a homogeneous water phantom for several electron beams of different energies, for $10 \times 10$ $\mathrm{cm}^{2}$ field size at $100 \mathrm{~cm}$ source-to-surface distance (SSD).

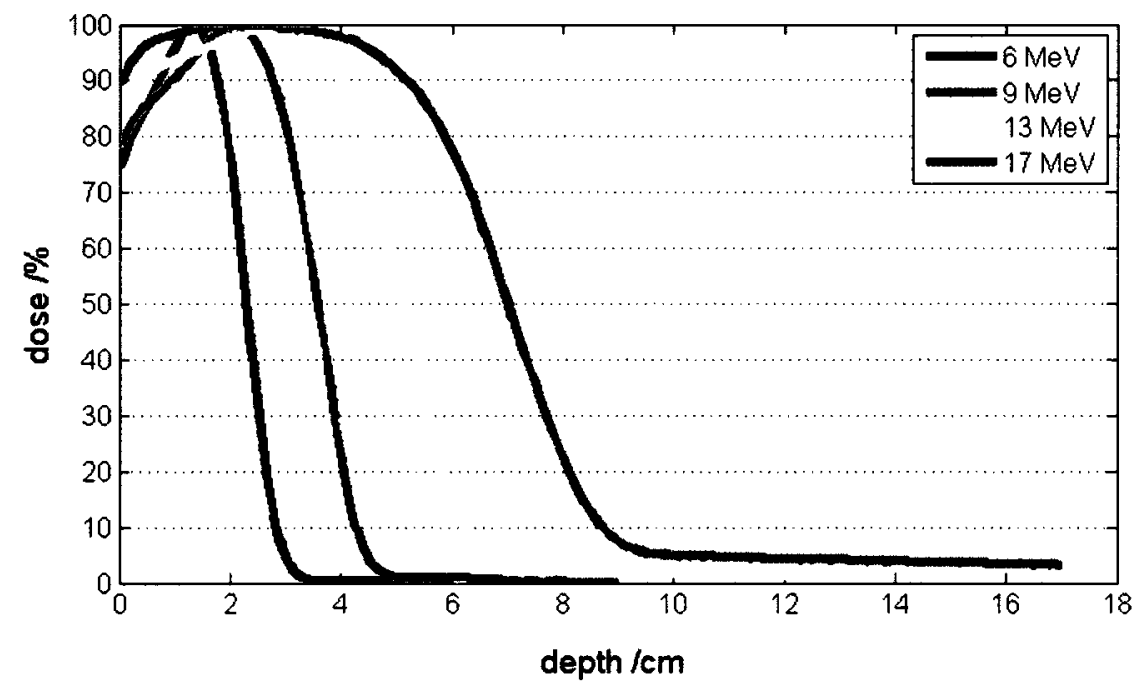

Figure 1. Normalized depth dose profiles of a $10 \times 10 \mathrm{~cm}^{2}$ beam at $100 \mathrm{~cm}$ SSD for electron beams of various energies.

The profiles can be characterized by several parameters that change with energy, and the density of the absorbing medium. The shape of the profile is due to the incident electrons losing their energy and stopping within the medium. For electron beams in a homogeneous phantom, the dose will build up until it reaches a maximum, $D_{\max }$, at which point the dose will quickly drop with depth. For lower energy beams, there is a sharper buildup (since the electrons scatter more), and the depth of maximum dose, $d_{\max }$, occurs closer to the phantom surface. For higher energies, the drop off of dose occurs at a larger depth. The tail at the end of the profile, termed the bremsstrahlung tail, is the dose that is due to the photons created in the head of the linac and in the phantom. The absorbed dose is partially due to primary electrons, secondary electrons, and bremsstrahlung. 
The range of the electrons can be described either by the half-value depth, $\mathrm{R}_{50}$, or the practical range, $R_{p} . R_{50}$ corresponds to the depth at which the absorbed dose falls to $50 \%$ of $\mathrm{D}_{\max }$, and can be approximated by the following relation ${ }^{13}$ :

$$
\bar{E}_{0}=C \cdot R_{50}
$$

where $\mathrm{C}=2.33 \mathrm{MeV} \cdot \mathrm{cm}^{-1}$ for water, and $\bar{E}_{0}$ is the mean energy of the incident beam at the surface of the phantom/patient. This equation is only valid for energies between 5-35 $\mathrm{MeV}$. The practical range describes the electrons that have traveled through the medium with the minimal amount of interactions. The practical range of an electron beam in a homogeneous water phantom can be approximated using the most probable energy at the absorbing medium surface, $\mathrm{E}_{\mathrm{P}, 0}$, and the following equation ${ }^{14}$ :

$$
R_{P}=0.53 E_{P, 0}-0.106
$$

where 0.53 has units of $\mathrm{cm} \cdot \mathrm{Mev}^{-1}, \mathrm{R}_{\mathrm{p}}$ has units of $\mathrm{cm}, \mathrm{E}_{\mathrm{P}, 0}$ has units of $\mathrm{MeV}$ and 0.106 has units of $\mathrm{cm}$. It is possible to approximate the mean energy of the electron beam at a particular depth of the profile using the practical range and the Harder equation ${ }^{15}$. The Harder equation ${ }^{15}$, Equation 3 , can be used to approximate the mean electron energy at a particular depth, $z(\mathrm{~cm})$, for a beam with initial beam energy $\bar{E}_{0}(\mathrm{MeV})$, and the practical range, $R_{p}(\mathrm{~cm})$.

$$
\overline{E_{z}}=\overline{E_{0}}\left[1-z / R_{p}\right]
$$

The equations above are approximations that hold true for percent depth dose (PDD) profiles in homogeneous water phantoms. In the case when the absorbing medium is not water, the depth can be scaled using the density of the medium, into what is known as a 
radiological depth ${ }^{16}$. Radiological depth is the depth a particle would need to travel

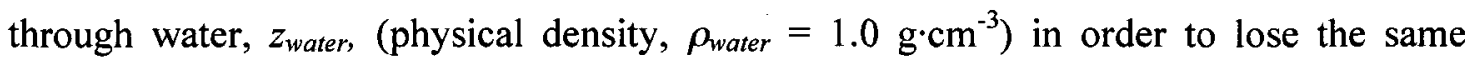
amount of energy as though it were traveling a depth, $z_{m e d}$, in a medium of density $\rho_{\text {med. }}$. It is calculated using the formulation ${ }^{16}$ presented in Equation 4.

$$
z_{\text {water }}=z_{\text {med }} \rho_{\text {med }} / \rho_{\text {water }}
$$

This principle can be applied to the half-value or practical range of an electron beam. For example, if the practical range in a homogeneous water phantom is known, the practical range in a medium with density $\rho_{\text {med }}$ can be calculated using:

$$
R_{p, \text { med }}=R_{p, \text { water }} \rho_{\text {water }} / \rho_{\text {med }}
$$

A dose profile is a result of many electrons losing energy over a distance. Typically the rate at which an electron loses energy is described as the average rate at which a beam of electrons lose energy. However, as individual electrons move through a medium, they do not lose energy at the same rate, or at a constant rate. This fluctuation of energy loss is a concept known as energy straggling ${ }^{17}$. The energy losses of individual electrons vary due to the stochastic nature of their interactions with the surrounding medium. Each electron will lose different amounts of energies at different points in the medium due to their interactions with the medium's orbital electrons and atomic nuclei. 


\subsection{Interactions and Energy Loss}

As high-energy electrons move through a medium, they interact with the medium's atomic nuclei and orbital electrons. These Coulomb force interactions are either elastic or inelastic, and can be classified as one of the following:

- Elastic collision with orbital electron

- Elastic collision with atomic nuclei

- Inelastic collision with orbital electron

- Inelastic collision with atomic nuclei

During an elastic interaction with an orbital electron, the orbital electron will move to a higher orbit before falling back into a lower orbit and releasing energy in the form of heat. During an elastic collision with the atomic nucleus, there is a change of the electron's original direction but no energy loss (elastic scattering). In an inelastic collision, the incident electron will lose energy in the form of radiative or collisional energy loss. When the incident electron interacts with the Coulomb field of the atomic nucleus, bremsstrahlung photons are produced; this is radiative energy loss. Collisional energy loss occurs when the incident electrons collide with the orbital electrons of the medium leading to the ionization or excitation of the atom. During ionization, knockon/secondary/ $\delta$-ray electrons are created. If the secondary electron has enough energy, it can continue on to ionize/excite other orbital electrons.

\subsubsection{Stopping Powers}

The rate at which electrons lose energy per unit length of a material is known as the linear stopping power and is typically expressed in units of $\mathrm{MeV} \cdot \mathrm{cm}^{-1}$. The mass stopping power is linear stopping power divided by density of the medium, expressed in 
units of $\mathrm{MeV} \cdot \mathrm{g}^{-1} \cdot \mathrm{cm}^{2}$. The total stopping power is a combination of the collisional and radiative energy losses.

$$
\left(\frac{d E}{d x}\right)=\left(\frac{d E}{d x}\right)_{C}+\left(\frac{d E}{d x}\right)_{R}
$$

The stopping power is dependent on a variety of factors, including the beam energy and the electron density (electrons $\mathrm{cm}^{-3}$ ) of the absorbing medium. Figure 2 presents the mass collisional stopping powers of several tissues over a range of energies. Within the range of energy used clinically for external electron beam therapy, the stopping power of water and water like materials can be approximated as $2 \mathrm{MeV} \cdot \mathrm{cm}^{-1}$ or $2 \mathrm{MeV} \cdot \mathrm{g}^{-1} \cdot \mathrm{cm}^{2}$.

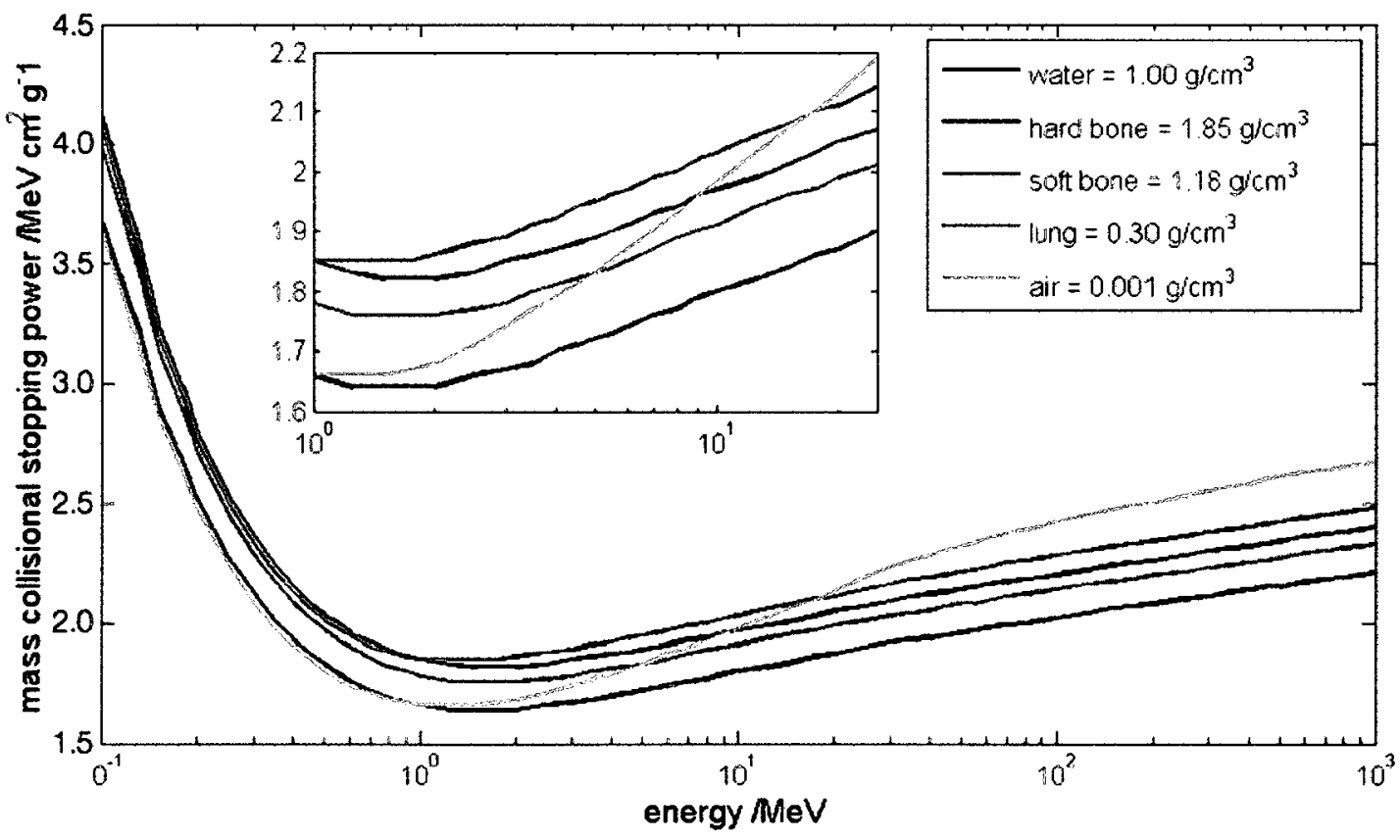

Figure 2. Collisional mass stopping powers of water, hard bone, soft bone, soft tissue, air and lung. Inset is a zoomed in view of the range of energies, $1-25 \mathrm{MeV}$. Values obtained using NIST ESTAR ${ }^{18}$. 
The linear stopping power is larger for those materials with a higher electron density; this is because there are more electrons within the absorbing medium with which the incident electrons can interact. The electron density is defined as the number of electrons per cubic centimeter. The electron density of a material is commonly expressed relative to the electron density of water, which is known as the relative electron density, RED. Since the electron density is expressed as a function of volume, it is also associated with the physical density of the material. Therefore, the physical density has an effect on the range the electron will travel through the medium. In other words, electrons would have to travel a shorter distance through a material of higher physical/electron density to lose the same amount of energy.

The total stopping power describes the energy lost by the incident particle due to collisional and radiative loses. The unrestricted stopping power ratio considers all energy loss to secondaries to be deposited locally. However, it is possible for secondary electrons, produced from inelastic collisions, to be sufficiently energetic to carry their kinetic energy far from the place of the original interaction. The energy lost by such electrons is not deposited locally. To account for this, a cut-off energy can be imposed so that only those secondaries with energy below the specified threshold are considered to deposit energy locally. Therefore, electrons above the energy threshold are considered as separate events. This rate of energy loss is termed the restricted stopping power ratio, and is lower than the unrestricted stopping power. 


\subsubsection{Mass Scattering Powers}

The incident electrons will undergo multiple scattering events as they move through the absorbing medium. This scattering is primarily due to Coulomb forces between the atomic nuclei of the medium and the incident beam. The mass scattering power, $T / \rho$, describes the mean square scattering angle per unit length of medium and unit density of medium $^{19}$. The mass stopping power is presented in Equation 7.

$$
\frac{T}{\rho}=\frac{1}{\rho} \frac{d \bar{\theta}^{2}}{d l}=\pi\left(\frac{2 r_{e} Z}{(\tau+1) \beta^{2}}\right)^{2} \frac{N_{A}}{M_{A}}\left\{\ln \left[1+\left(\frac{\theta_{\max }}{\theta_{\min }}\right)^{2}\right]-1+\left[1+\left(\frac{\theta_{\max }}{\theta_{\min }}\right)^{2}\right]^{-1}\right\}
$$

where $r_{e}=$ classic electron radius, $Z=$ atomic number, $\beta=$ velocity of the incident electron relative to velocity of light in a vacuum, $N_{A}=$ Avogadro constant, $M_{A}=$ molar mass of absorbing medium, $\theta_{\max }=$ cut off angle for large deflections, $\theta_{\min }=$ screening angle for small deflections, $\tau=$ the ratio of the kinetic energy to the rest energy of the electron, $l=$ length, and $\rho=$ density of the material.

As the beam of electrons moves through the medium, it loses energy and the angular spread of the beam increases. This occurs because the scattering power is inversely proportional to the energy of the incident electrons. The scattering power also increases as the square atomic number. 


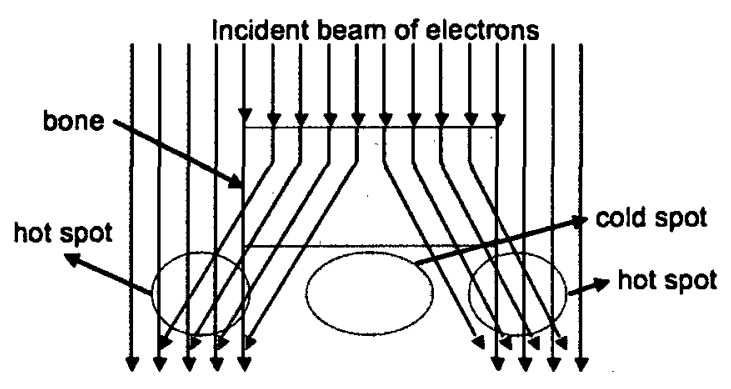

Figure 3. Presence of hot and cold spots can be visible in the presence of small inhomogeneities such as hard bone in water. This drawing was adapted from Khan ${ }^{20}$.

When a small inhomogeneity is present, with a higher scattering power than the medium it is in, electrons will be scattered at larger angles, increasing the fluence around it, and decreasing the fluence behind it. This variation of the scattering power disrupts the side scatter equilibrium thus creating volumes of elevated dose (hot spots) or lesser dose (cold spots), shown in Figure 3. The dose in a small volume with a higher scattering power will be smaller since the electrons are more likely to scatter, irradiating away a portion of its original energy. Hot spots can also happen if there are surface inhomogeneities. In these situations a bolus can be introduced to smooth out irregular edges.

\subsubsection{Dose: The absorption of energy}

Absorbed dose is the energy deposited by ionizing radiation into the absorbing medium per unit mass. The measure of dose is typically in units of Joules per kilogram, more commonly referred to as "gray", Gy. Another way of defining dose is by calculating the number of particles that are incident on a volume multiplied by the amount of energy they deposit. 


$$
D=\int_{\Delta}^{E_{\max }} \Phi_{E}(E)\left(\frac{L(\Delta)}{\rho}\right) d E+T E
$$

The differential charged particle fluence (number of particles per unit energy at energy E), $\Phi_{E}$, is multiplied by the restricted mass stopping power ratio, $L(\Delta) / \rho$. By using the restricted mass stopping power, we are only considering secondary electrons with energy above the threshold energy $\Delta$. This definition of dose follows the Spencer-Attix theory since secondary electrons above threshold energy $\Delta$ are accounted for in the fluence. The first term of ( 8 is integrated over the spectrum of energies for the incident electrons above $\Delta$; the dose deposited by electrons below the energy threshold is accounted for in the track end term, TE. The last term takes into consideration dose deposited by those electrons whose energy falls below the threshold energy $\Delta$ and because of this they will stop within the volume. 


\section{Chapter 3}

\section{Theraplan Plus $\circledast$ : A Monte Carlo based treatment planning system}

For the purpose of our analysis, we have been using Theraplan Plus ${ }^{\circledR}$ v.3.8 and DCM v.2.0. Theraplan Plus $\AA^{\circledR}$, developed by MDS Nordion (Ottawa), presently Nucletron, uses a $\mathrm{VMC}++$ dose engine to calculate three dimensional dose distributions in a medium. The software for this treatment planning system is based on Kawrakow's VMC++ algorithm $^{21,22,23}$, which uses a voxel Monte Carlo-based algorithm to simulate/predict the transport of particles through a voxelised phantom or patient medium. This new approach has many advantages; it requires much less computational time than any other algorithm of its type and needs much less pre-calculated data.

\subsection{Anatomy Modeling}

Treatment plans can be generated for vector or image-based anatomies. Vector-based anatomies, also known as mathematical phantoms, are anatomies with well-defined boundaries and bulk relative electron densities. Using the Anatomy Modeling module of the Theraplan Plus ${ }^{\circledR}$ Treatment Planning System, these well-defined anatomies can be designed to incorporate high and low density inhomogeneities of varying complexities. To create a vector anatomy, the user is required to specify the number and thickness of the slices in the transverse plane. The program displays the individual slices so that the user can then create contours around regions of interest on each slice. A region can be delineated by either drawing the contour with a cursor, selecting and placing a predefined shape from a library, or entering the contour coordinates. For this work, all contours were created by defining the coordinate points. In the case of vector anatomies, 
inhomogeneities are created using contours, whereas in patient anatomies contours are used to define boundaries on preexisting inhomogeneities. Typically, for image-based anatomies, the organs at risk and tumors are delineated. Subsequent contours are delineations of volumes within the external contour; these are useful for defining volumes for which the user will require dose volume histograms, DVH. For vector-based anatomies, each contour must be assigned an anatomical label and corresponding RED. The user may choose from the anatomical dictionary provided or create a new label and RED.

It is also possible to import an image-based anatomy. Typically, the imported image is a CT scan of the patient. The slices and their thicknesses are determined by the properties of the CT dataset. Once imported, a physician will contour the tumor and organs at risk. Unlike the vector anatomy, the organs are not assigned bulk relative electron densities. The REDs and tissue types are assigned based on the Hounsfield (HU) number of the pixels in the image. Once an RED is assigned, it is matched with one of the predefined tissues listed in the dose calculation module, DCM. The Monte Carlo dose calculation module in Theraplan Plus ${ }^{\circledR}$ has 21 different tissues to choose from, making the material assignment of the pixel more accurate than in those TPS with a smaller tissue inventory.

\subsection{Creating a treatment plan and viewing dose calculations}

Once the anatomy is contoured it is exported into the External Beam Planning module of Theraplan Plus $®$, where the treatment plan is designed. The program requires the beam information including, but not limited to, beam energy, gantry angle, SSD, field size, and 
the application of inhomogeneity corrections. At this point in the planning process, the user can also specify the size of the display matrix and dose calculation points of interest. The display matrix is a three-dimensional array of points for which the calculated dose is displayed for each transverse slice. The display matrix is defined by the spatial limits in the two perpendicular directions (slice thickness), and the point resolution in each direction of the transverse plane (this is especially important for image-based anatomies). The prescription dose or number of monitor units (MU) to be delivered is entered and the plan is then sent to the DCM, for Monte Carlo calculations.

Once the plan has been calculated, the user can analyze it with one of the various tools provided by Theraplan Plus ${ }^{\circledR}$. The user can view isodose distributions, generate the cumulative or differential dose volume histograms and view 3D images of the contoured anatomy and dose distributions. Within the External Beam Planning module, the display matrix dose values can be seen (or extracted manually) for one image slice at a time. DVHs of previously contoured structures can also be obtained from the treatment planning system. Due to the limitations of the program, calculation uncertainties cannot be directly obtained from this version of the program.

\subsection{DCM: Dose Calculations Module}

The dose calculation module, DCM, has two main tasks, each implemented with different engines $^{24}$ :

1) The reconstruction of the beam phase space

2) Particle transport and dose calculation 


\subsubsection{Beam Phase Space}

To cut down on calculation time, the phase space is not reconstructed for every calculation. Instead, the beam is modeled and the prepackaged exit phase space is provided for every possible combination of applicator size and beam energy.

The beam is modeled at two points, before the first changeable collimating component, and after the last collimating component; these phase spaces are known as the source phase space and exit phase space respectively. The phase space contains a sequence of particles; each particle has an assigned energy, charge, position and direction.

Dose engines are dependent upon the relative particle fluence distribution. This fluence can be divided into three categories: primary electrons, indirect electrons and photon fluence. The primary fluence is made up of direct electrons that propagate through the linac head without interacting with any of the collimating components. The indirect fluence is defined as the electrons that hit collimating components of the machine head. These electrons are scored for each collimating element, and then using pre-calculated scattering kernels, only those secondaries with a clear path to the patient are scored in the exit phase space. The secondaries from each collimator that are eventually scored in the exit phase space, this is referred to as multi-source fluence. Finally, the photon fluence is from any bremsstrahlung interactions in the treatment head (photon fluence created within the patient is taken into account during the in-patient calculation). All forms of fluence are saved into 2D matrices and wielded during patient dose calculations ${ }^{24}$. 


\subsubsection{In-Patient Dose Calculations}

Although the user can define a display matrix to see the calculated dose, the dose is actually calculated for and stored in a matrix known as the calculation matrix. Dose is calculated at the center of each calculation matrix voxel, and then linearly interpolated into the display matrix and any predefined calculation points ${ }^{3}$. Calculation matrices are created for image and vector-based anatomies. The user has no control of the dimensions of the calculation matrix voxels. Rather it is selected by the program based on the constraint that the number of voxels in any slice does not exceed 16,384 , or 2,048 along any $\operatorname{axis}^{3,24}$. The calculation matrix does not align with the display matrix defined by the user during the treatment planning process. This is for two main reasons: (1) the dimensions of the calculation and display matrices typically do not agree and (2) the minimum xyz coordinates are not defined at the same point with respect to the anatomy. A pixel of the calculation matrix can contain several pixels (not necessarily whole) of the display matrix, as shown in Figure 4.

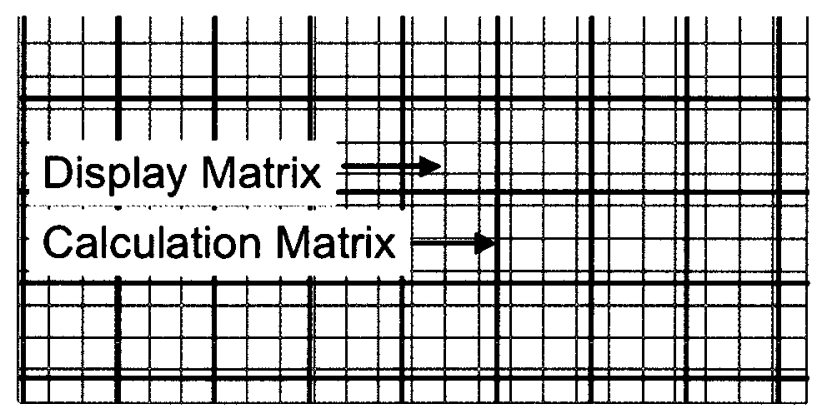

Figure 4. The user defined display matrix (shown in green), does not necessarily align with the calculation matrix (shown in black) defined by the algorithm.

Prior to calculation, the image is divided into the calculation voxels. Using the average 
of the HU values of the image pixels contained within a calculation matrix pixel, the calculation voxel is assigned tissue properties. This process can degrade the resolution of the anatomy since the averaging of image pixel HU values can result in blurring of inhomogeneity boundaries. The larger grid in Figure 5 represents the calculation matrix, and the smaller grid is representative of the image pixels (for clarity, the display matrix is not shown). Figure 5 (a) is an example of an anatomy slice containing an inhomogeneity with the calculation matrix superimposed on it prior to assigning material-types to the calculation matrix. In Figure 5 (b), the voxels that are fully shaded have been assigned the appropriate material-type. From the figure, it can be seen that voxels containing the inhomogeneity were not necessarily assigned the correct material-types due to the averaging of the pixel values. 


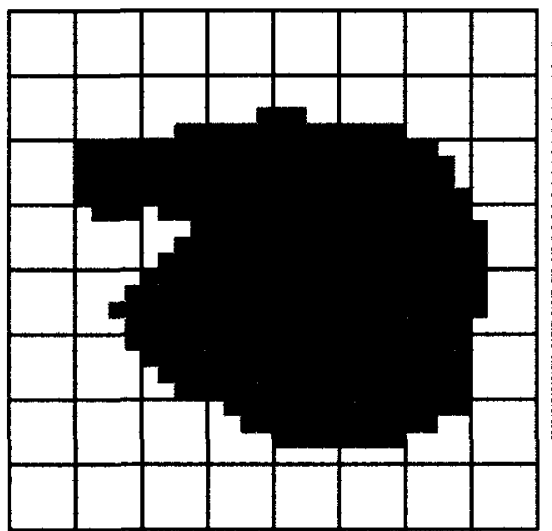

(a)

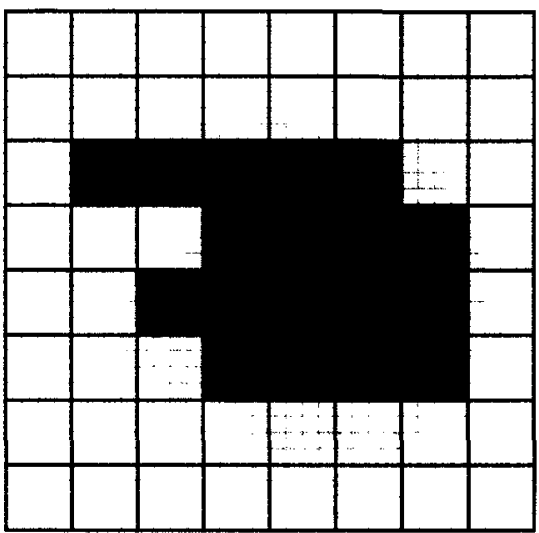

(b)

Figure 5. Voxels in the calculation matrix are assigned material properties based on the average $H U$ numbers within the voxel. The larger grid represents the pixels of the calculation matrix, the smaller grid represents the pixels of the phantom/patient image. For clarity, the display matrix is not shown. The image to the left is an example of an inhomogeneity with the calculation matrix superimposed on it prior to averaging the $H U$ values of the image pixels. In the image to the right, the voxels that are fully shaded have been assigned the proper material properties of the inhomogeneity.

The edges of the inhomogeneity will be slightly distorted due to the method in which the RED values are assigned. These effects should be small since the voxel sizes used for this study were smaller than $0.4 \mathrm{~cm}$ in all directions. The user cannot view the material type (or average $\mathrm{HU}$ ) assigned to voxels of the calculation matrix.

Once the calculation matrix is constructed, individual particles are sampled from the exit phase space and transported through the medium. As a particle is transported through the medium, the program must determine the angle and energy of each particle and the distance to its next interaction. These parameters are determined by randomly sampling from probability density functions. The energy deposited in each calculation voxel is calculated by multiplying the distance traveled by the electron in the voxel by the stopping power of the electron within the medium of the voxel. When a particle interacts 
with the medium in a voxel several things can occur:

1) The particle that is created has enough energy that it is not absorbed in the calculation voxel. The particle's energy and direction are sampled and later it will be tracked separately through the medium until it is absorbed.

2) The interaction creates a particle with kinetic energy below the energy cutoff threshold. The history of the particle is terminated and the energy is scored for that voxel.

3) The interaction creates a particle that is absorbed in the medium (the particle does not have enough energy to exit the voxel); in this case, the particle's energy is scored.

Figure 6 illustrates the sequence of steps during the dose calculation process. It is important to note that some of these steps are medium dependent. 


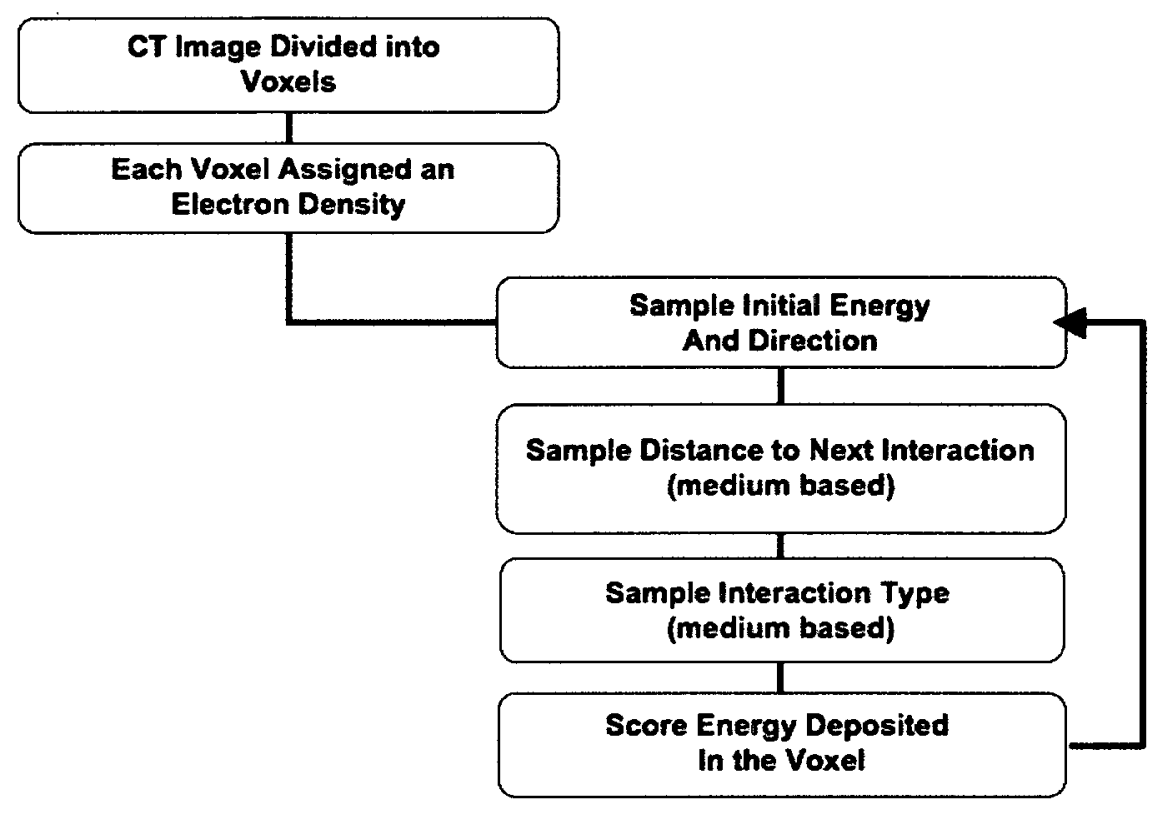

Figure 6. Workflow of the Monte Carlo simulation for each particle.

During the validation of the Theraplan Plus ${ }^{\circledR}$ software, Cygler et al ${ }^{25}$ found the calculations to have an overall uncertainty of less than $1.5 \%$. This uncertainty was established for plans with 50,000 histories per square centimeter of the irradiated surface. The accuracy improves as the number of histories per square centimeter is increased, however, if the number of histories remains the same and the voxel size decreases the overall uncertainty will increase $\mathrm{e}^{25}$. This is due to the fewer number of interactions within the voxel. Unfortunately, the user has no control over the size of the voxels, and calculation uncertainties cannot be directly obtained from Theraplan Plus ${ }^{\circledR}$. 


\section{Chapter 4}

\section{Dose-to-water vs. dose-to-medium}

In $\mathrm{VMC}++$ the dose is calculated in terms of $\mathrm{D}_{\mathrm{m}}$. During the transport of the electron through a medium, the distance between interactions and the interaction types depend on the medium through which the electrons are traveling. Currently there is no consensus whether dose should be calculated and reported in terms of $D_{m}$ or $D_{w}{ }^{8}$. In conventional treatment planning systems, CT numbers are converted to electron densities of water-like material before calculations are performed. The Monte Carlo based treatment planning systems have the ability to calculate $D_{m}$. Although it is possible to convert the $D_{m}$ values to $\mathrm{D}_{\mathrm{w}}$, this is unfavorable since it introduces additional uncertainties to the dose distribution.

It is a topic of much debate in the medical physics community whether one of these two approaches $\left(D_{w}\right.$ vs. $\left.D_{m}\right)$ is more appropriate. In a Point/Counterpoint article ${ }^{8}$, Keall debated for preserving the $D_{w}$ approach in clinical dose calculations. His argument was based on the fact that absolute dosimetry protocols and all clinical experience up to now are based on $\mathrm{D}_{\mathrm{w}}$ calculations. In the same article ${ }^{8}$, Liu presented the opposing view, noting that she did not expect the switch between $D_{w}$ to $D_{m}$ to have a major effect in radiotherapy. Lui argument stated that calculating $D_{m}$ would result in improved accuracy of patient dosimetry.

Clearly, a thorough analysis of the differences between treatment plans calculated for $D_{m}$ and $D_{w}$ is needed. It will promote an understanding of whether there is a clinically significant difference between the two calculation methods. Such an analysis has already been carried out for clinical cases using photon beams. In a clinical study for IMRT 
prostate and head and neck patients, Dogan et al ${ }^{26}$ found that for prostate, and head and neck patients there were systematic differences between the $D_{m}$ and $D_{w}$ dose-volume indices of up to $2.9 \%$ and $3.5 \%$, respectively, for the prescription dose to the PTV. Siebers et $a l^{27}$ found that differences between $D_{m}$ and $D_{w}$ calculations range from $1 \%$ for soft tissues to $10 \%$ for cortical bone for photon beam dose calculations. He proposed using the unrestricted mass stopping power ratio (SPR) of $D_{m}$ as a post-processing multiplicative factor to convert $D_{m}$ to $D_{w}$ for photon beams.

$$
D_{\text {water }}=D_{\text {medium }}\left(\frac{S}{\rho}\right)_{\text {medium }}^{\text {water }}
$$

Figure 7 shows the ratio of water-to-medium unrestricted mass stopping power ratios for several tissues. The mass collisional stopping power of lung varies from being $1 \%$ higher to almost $4 \%$ lower than unity, and for cortical bone it can be almost $14 \%$ higher than unity. Within the energy range used in external beam electron radiotherapy, the stopping power ratios (except for air) are roughly constant. In the energy range of 2 to $25 \mathrm{MeV}$, the mass collisional stopping power of water to hard and soft bone are larger than water by approximately $9.8 \%$ and $3.3 \%$, respectively. For lung, this ratio is approximately $1.5 \%$ lower than unity. 


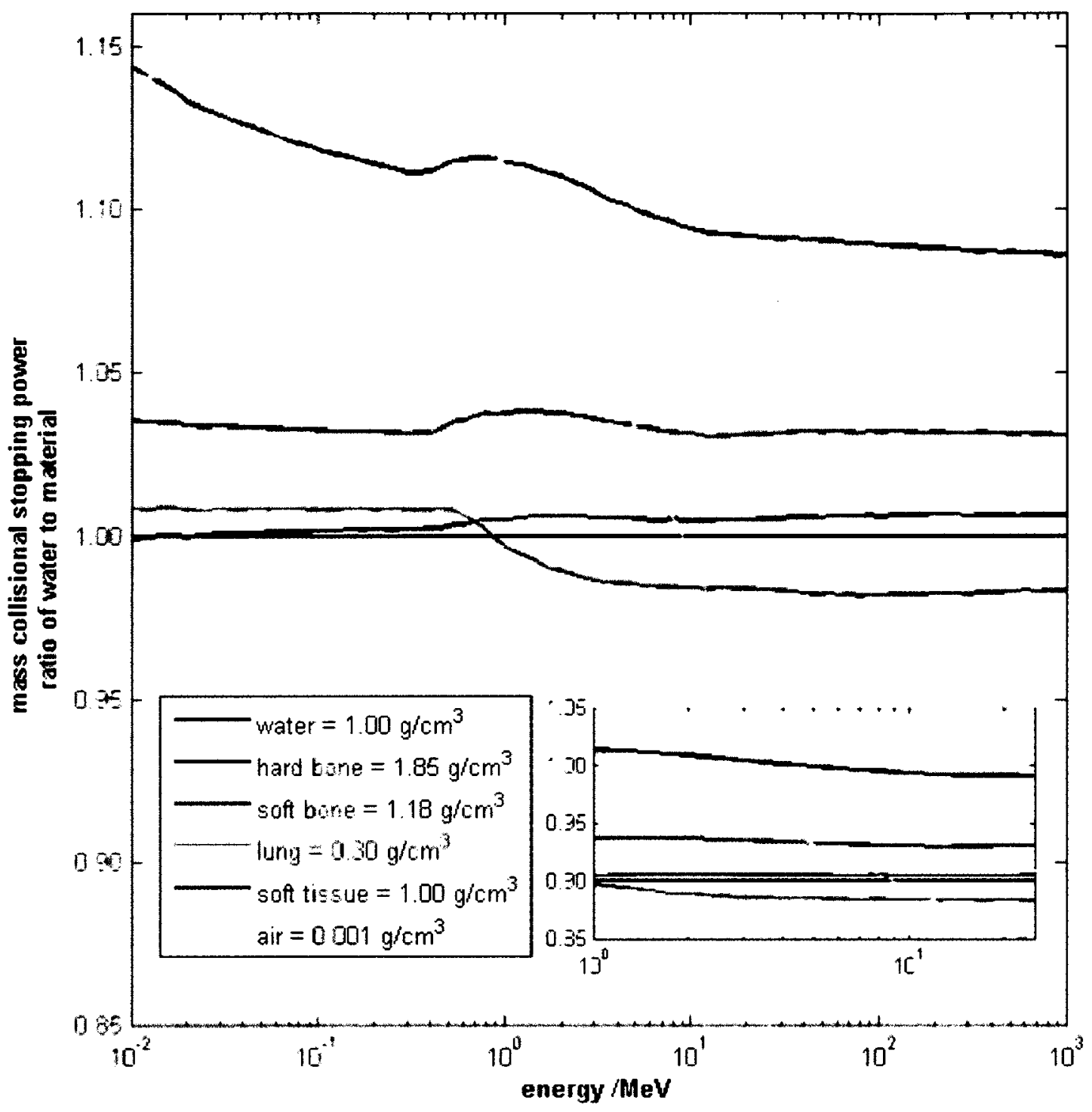

Figure 7. Ratio of unrestricted mass collisional stopping power ratios of water to material. Values were obtained from NIST ESTAR ${ }^{18}$ for mono-energetic electrons of energies 0.01 to $1000 \mathrm{MeV}$. Inset is a zoomed view of the range of energies.

$\mathrm{VMC}^{++}$converts $\mathrm{D}_{\mathrm{m}}$ to $\mathrm{D}_{\mathrm{w}}$ on a track-by-track basis rather than as a post-processing step $^{28}$. The energy deposited by an electron in a voxel is multiplied by the ratio of collisional stopping powers. These values are evaluated at a random energy within the 
range of the initial and final energy of the electron for that step.

Systematic studies of the dosimetric differences between the $D_{m}$ and $D_{w}$ such as the ones described in this chapter do not exist for Monte Carlo based electron beam dose calculation. The purpose of our study was to calculate dose distribution with a commercial MC based treatment-planning system, Theraplan Plus ${ }^{\circledR}$, using $D_{w}$ and $D_{m}$ approaches and compare the dosimetric differences between such plans for well defined phantom anatomies as well as patients. 


\section{Chapter 5}

\section{Proof-of-Concept Phantoms}

\subsection{Proof-of-concept phantom configurations}

To determine whether any differences exist in the simplest scenario, plans were created and calculated for three vector-based phantoms, each containing a different inhomogeneity. The plans were created in the Anatomy Modeling module of Theraplan Plus ${ }^{\circledR}$ using 61 transverse slices, $0.5 \mathrm{~cm}$ thick. Each phantom had external dimensions of $30.0 \times 30.0 \times 12.0 \mathrm{~cm}^{3}$, and an RED of 1.00 . These served as template blocks of tissue, representative of the anatomy within the external contour. Each of the three phantoms contained a different $30.0 \times 30.0 \times 1.0 \mathrm{~cm}^{3}$ slab of inhomogeneity positioned $1.0 \mathrm{~cm}$ below the phantom surface.

Figure 8 is a depiction of a sample phantom. The black wire frame is the external contour, the blue slab is representative of the slab of inhomogeneity within the phantom, and the green rectangle is the location of the beam incident on the phantom.
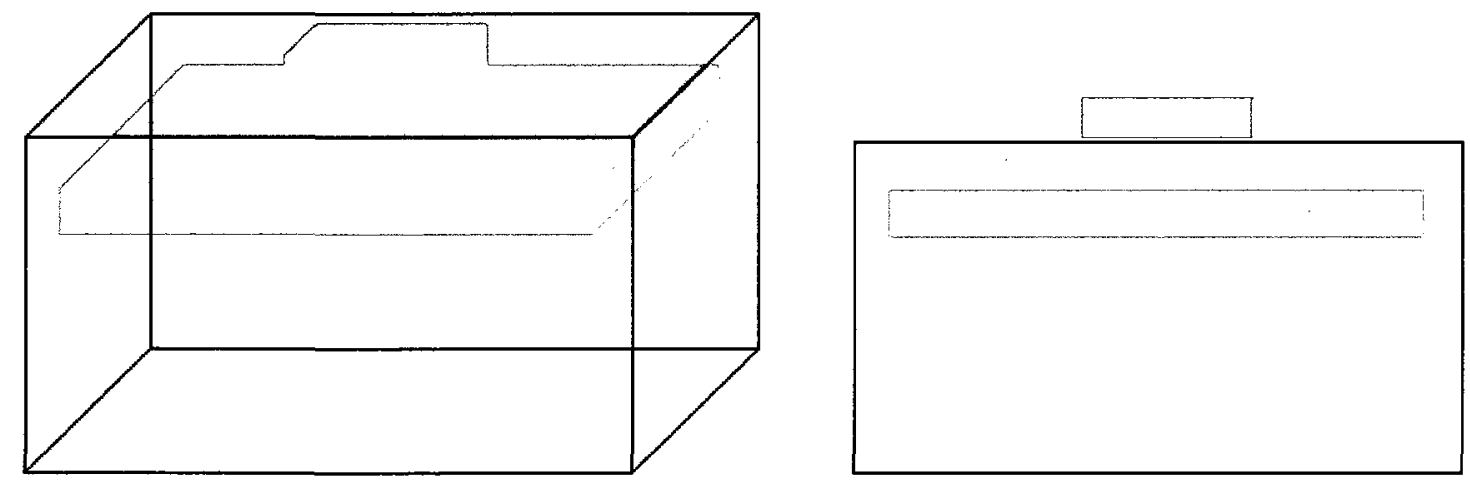

Figure 8. Structural depiction of the proof of concept mathematical phantoms. The external anatomy is outlined in black, while the contoured inhomogeneity is shown in solid blue. The green cube delineates the external (electron) beam. The central transverse slice is shown to the right. 
Phantoms 1, 2 and 3 contained a lung, soft bone and hard bone tissue-substitute slab, respectively. Each inhomogeneity was assigned a bulk electron density similar to a tissue found within the body. These tissues were chosen to represent a broad range of tissue densities found within the human body. The bulk REDs assigned to slab inhomogeneities are based on the REDs typically observed in patient anatomies. These densities have been summarized in Table 1.

Table 1 Relative electron densities used for proof-of-concept mathematical phantoms as seen in preliminary patient plans.

\begin{tabular}{lc}
\hline Tissue & $\begin{array}{c}\text { Relative Electron } \\
\text { Density }\end{array}$ \\
\hline \hline Water & 1.00 \\
Lung & 0.30 \\
Soft Bone & 1.15 \\
Hard Bone & 1.74 \\
\hline
\end{tabular}

Two plans were created for each phantom. The first plan used a $9 \mathrm{MeV}$ beam and the second used a $17 \mathrm{MeV}$ beam. These energies were chosen to represent the differences that can occur at either end of the range of energies used in external electron radiotherapy. Other than the energy, both plans were identical. The beams were modeled to be perpendicularly incident on the center of the phantom surface, had a $10.0 \mathrm{x}$ $10.0 \mathrm{~cm}^{2}$ field size and a $100 \mathrm{~cm}$ SSD. The display matrix was set to $0.2 \times 0.2 \times 0.2 \mathrm{~cm}^{3}$. With inhomogeneity corrections turned on, the plans were calculated to deliver $100 \mathrm{MU}$. At The Ottawa Hospital Cancer Center, $100 \mathrm{MU}$ corresponds to $100 \mathrm{cGy}$ at $\mathrm{d}_{\max }$ in a homogeneous water phantom. Both plans were calculated twice, once using the $D_{m}$ approach, and then again using the $\mathrm{D}_{\mathrm{w}}$ approach. All calculations were done using the Monte Carlo option in Theraplan Plus $\left({ }^{\circledR}\right.$ Dose Calculation Module. 


\subsection{Calculations for proof-of-concept phantoms}

The purpose of the preliminary phantom work was to show whether differences exist in the most basic geometrical scenario between the $D_{m}$ and $D_{w}$ calculated plans and whether the ratio of these calculations is in agreement with mass stopping power ratios.

Following the approach by Siebers $e t a l^{27}$, the unrestricted mass collisional stopping powers were evaluated at the depths of maximum difference for each phantom and energy. The mass stopping power ratios were obtained from NIST ESTAR ${ }^{18}$ using the elemental compositions outlined in the papers by White $e t a l^{29,30}$, and the mean electron energies evaluated using the Harder ${ }^{15}$ approximation. For the purpose of comparison, differences are expressed as a ratio of $D_{w}$ to $D_{m}$.

The proof of concept phantoms presented in this chapter have $0.39 \times 0.39 \times 0.39 \mathrm{~cm}^{3}$ voxel size, and were calculated with 50,000 electron histories $\cdot \mathrm{cm}^{-2}$. The uncertainty of the Monte Carlo calculations for an anatomy with this voxel size and number of incident histories has an uncertainty of less than $2 \%{ }^{25}$.

\subsubsection{Results: Lung slab phantom}

The largest difference in the lung phantom between the $D_{m}$ and $D_{w}$ calculations was $1.6 \%$ for the 9 and $1.7 \%$ for the $17 \mathrm{MeV}$ plans. For the $9 \mathrm{MeV}$ plan, this difference occurred at a depth of $1.4 \mathrm{~cm}$, within the lung slab, see Figure 9 (a). At this depth, the mean electron energy is $6.84 \mathrm{MeV}$, which corresponds to the water to lung mass collisional stopping power ratio of 0.97 ( $3 \%$ below unity).

Figure 9 (b) displays the central axis profile of the lung phantom when a $17 \mathrm{MeV}$ beam is used to deliver the planned dose. The maximum difference occurs at a $1.4 \mathrm{~cm}$ from the 
phantom surface, within the lung slab. The water to lung mass SPR within the lung evaluated at the mean electron energy, $14.86 \mathrm{MeV}$, at the depth of maximum difference is 0.97 .

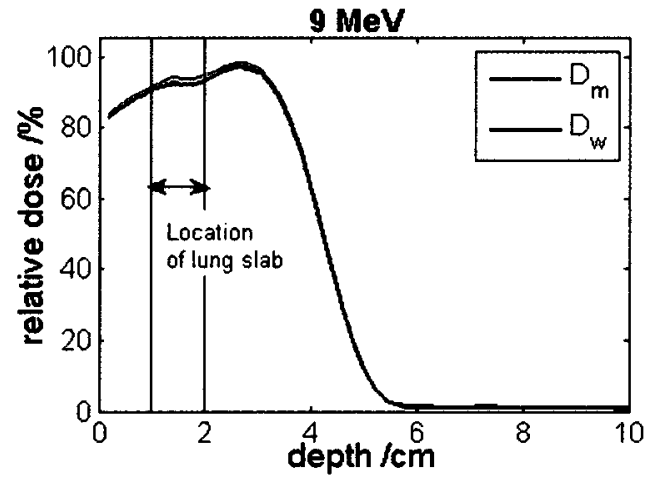

(a)

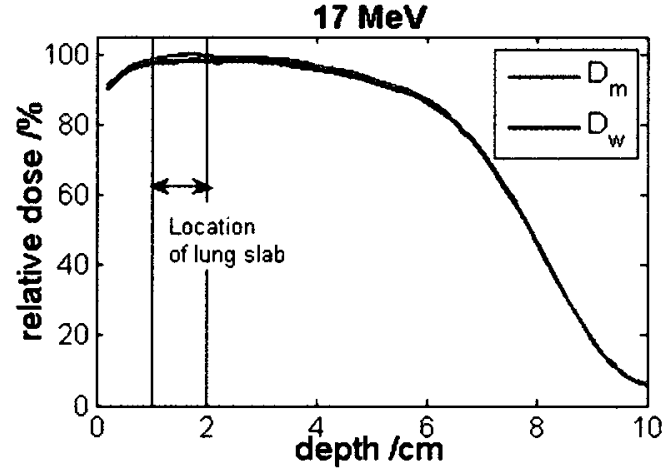

(b)

Figure 9. Axial dose profiles along the central axis of the proof-of-concept lung phantom irradiated with a (a) $9 \mathrm{MeV}$ beam, (b) $17 \mathrm{MeV}$ beam. Profiles were normalized to $D_{\max }$ in a homogenous water phantom.

The dose within the lung is larger when calculated using the $D_{m}$ approach for both energies. The $D_{w} / D_{m}$ ratios at the depths of maximum difference were in reasonable agreement with the mass collisional stopping power ratios evaluated at those depths.

\subsubsection{Results: Soft bone slab phantom}

The central axis profiles for the 9 and $17 \mathrm{MeV}$ soft bone phantom calculations are shown in Figure 10 (a) and (b). The largest difference between the $D_{m}$ and $D_{w}$ profiles for the 9 and $17 \mathrm{MeV}$ plans was $1.6 \%$ for both plans. Dose-to-water is larger than dose-tomedium for both plans. The maximum difference between the $9 \mathrm{MeV} \mathrm{D}_{\mathrm{m}}$ and $\mathrm{D}_{\mathrm{w}}$ profiles occurred within the soft bone slab, $1.6 \mathrm{~cm}$ from the phantom surface. Using the 
mean electron energy at this depth, $5.74 \mathrm{MeV}$, the mass collisional stopping power ratio within the soft bone is 1.03 .

When a $17 \mathrm{MeV}$ beam was used to deliver the prescribed dose of $100 \mathrm{cGy}$, the maximum difference also occurred at $1.6 \mathrm{~cm}$ from the surface, within the soft bone slab. At this depth, the mean electron energy is $13.77 \mathrm{MeV}$, and which corresponds to a mass collisional stopping power ratio of 1.03. The mass collisional stopping power ratios are subject to uncertainties based on the approximations made regarding the energy at the surface and the depth of interest, as well as the error associated with the stopping power ${ }^{18}$ itself. Based on the uncertainty of the values that are being compared it can be stated that the mass collisional stopping power ratios within the soft bone slab were reasonably consistent with their corresponding $\mathrm{D}_{\mathrm{w}} / \mathrm{D}_{\mathrm{m}}$ for both the 9 and $17 \mathrm{MeV}$ plans.

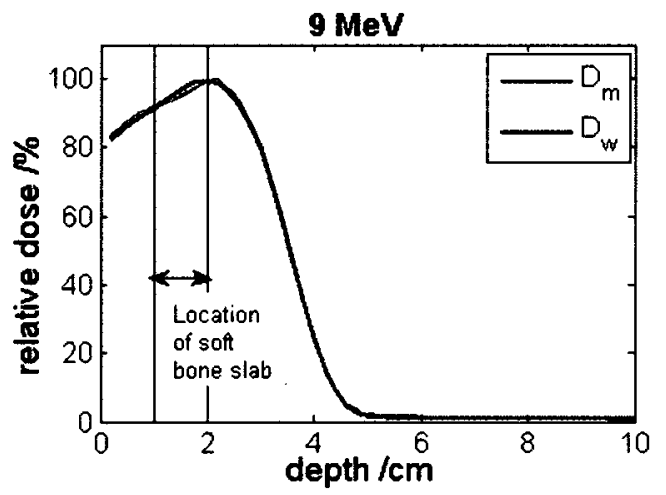

(a)

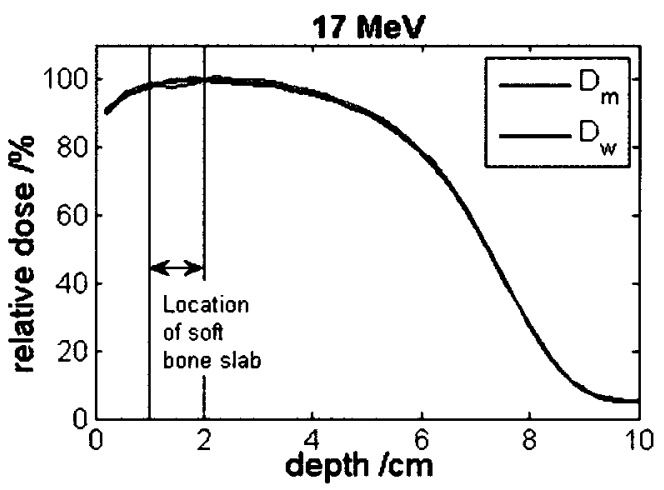

(b)

Figure 10. Axial dose profiles along the central axis of the proof-of-concept soft bone phantom irradiated with a (a) $9 \mathrm{MeV}$ beam, (b) $17 \mathrm{MeV}$ beam. Profiles were normalized to $D_{\max }$ in a homogenous water phantom. 


\subsubsection{Results: Hard bone slab phantom}

The hard bone phantom central axis profiles are presented in Figure 11 (a) and (b). Similar to the soft bone slab, the differences between $D_{m}$ and $D_{w}$ were larger for the 9 $\mathrm{MeV}$ plan. The largest difference between $D_{m}$ and $D_{w}$ profiles for the 9 and $17 \mathrm{MeV}$ plans were $11.3 \%$ and $9.8 \%$, respectively. The greatest difference between the $9 \mathrm{MeV}$ profiles occurred within the bone, at a depth of $1.6 \mathrm{~cm}$ from the phantom surface. At this depth, the mean electron energy is $4.93 \mathrm{MeV}$. The mass collisional stopping power ratio of water to bone was calculated for this energy was $10 \%$ above unity.

The largest difference between $D_{m}$ and $D_{w}$ for the plans calculated with the $17 \mathrm{MeV}$ beam was located shallower than that of the $9 \mathrm{MeV}$ plans: it occurred $1.4 \mathrm{~cm}$ from the surface. The SPR at this depth was $9 \%$ above unity, which was evaluated at $12.97 \mathrm{MeV}$ (the mean electron energy this depth).

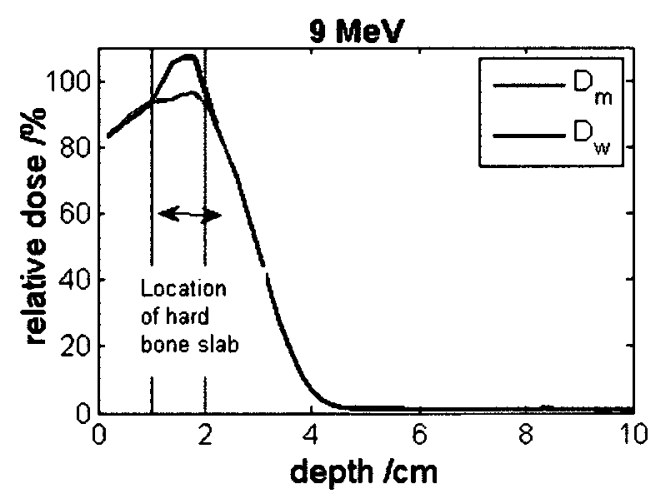

(a)

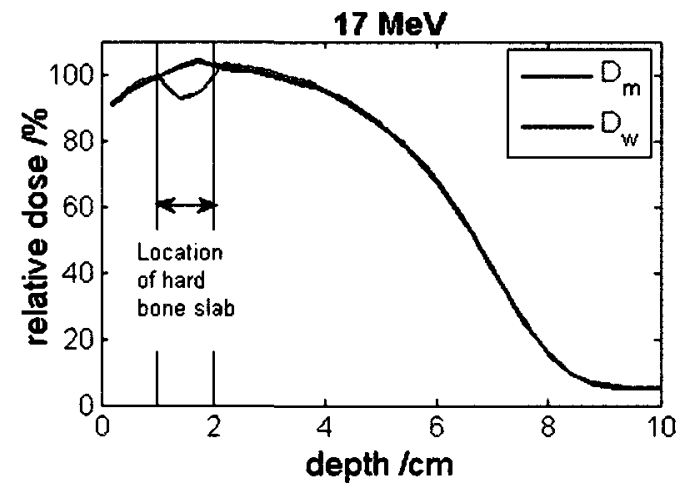

(b)

Figure 11. Axial dose profiles along the central axis of the proof-of-concept hard bone phantom irradiated with a (a) $9 \mathrm{MeV}$ beam, (b) $17 \mathrm{MeV}$ beam. Profiles were normalized to $D_{\max }$ in a homogenous water phantom.

As expected, results from this preliminary study showed differences between the $D_{m}$ and $D_{w}$ calculation approaches. We see larger differences between $D_{m}$ and $D_{w}$ calculations for 
plans using lower beam energy. These differences become more exaggerated as the RED of the heterogeneity diverges further from unity. For the basic geometries shown, $\mathrm{D}_{\mathrm{w}}$-to$D_{m}$ ratios were consistent with the water-to-medium mass collisional stopping power ratios evaluated at the depth of interest. Based on these results we continued our investigation using epoxy resin based tissue substitute phantoms to quantify these differences for different inhomogeneity geometries, as well as within patient plans. 


\section{Chapter 6}

\section{Epoxy resin based phantoms}

Following the preliminary proof-of-concept studies using mathematical phantoms, physical phantoms were used to mimic the anatomies of interest and to compare the calculations with experimental results. These were constructed by stacking several epoxy resin-based (ERB) tissue substitute slabs (from hereon referred to as tissue-substitute slabs), mimicking various tissues in the body (i.e. muscle, bone, and lung), in different geometrical configurations. The use of these phantoms and their description has been well published before ${ }^{31,32,33,34}$.

\subsection{Epoxy resin based phantom configurations}

The tissue-substitute slabs were of varying geometries, densities and complexities as listed below:

- Slab Geometry - One dimensional (1D)

- Small Cylindrical Geometry - Three Dimensional (3D)

- Trachea and Spine - Complex Geometry (low and high inhomogeneities)

Five phantoms were used, each with a different inhomogeneity and geometry: bone slab, lung slab, bone cylinder, air cylinder, and trachea and spine. The phantoms were milled at the University of Wisconsin from GAMMEX RMIß tissue equivalent materials ${ }^{10,31,34}$. The physical and relative electron densities of these materials are based on the elemental composition of the epoxy resin based tissue substitute material described by White et $a l^{29,30}$ and information from RMI. These values have been summarized in Table 2. The phantoms consisted of several stacked tissue-substitute (MS11) slabs. The stacked slabs 
were placed above an assembly of four MS11 slabs, referred to as the backscatter assembly. The MS11 material has similar physical density, absorption, scattering and attenuation characteristics as muscle. The two slabs of the backscatter assembly were 5.0 and $6.0 \mathrm{~cm}$ thick, for a total thickness of $11.0 \mathrm{~cm}$. Each slab, with the exception of the backscatter assembly slabs, had lateral dimensions of $15.0 \times 15.0 \mathrm{~cm}^{2}$. The backscatter assembly slabs had lateral dimensions of $30.0 \times 30.0 \mathrm{~cm}^{2}$.

Table 2 Physical and relative electron densities of the epoxy resin based tissue substitute materials, adapted from White et al ${ }^{29,30}$.

\begin{tabular}{|c|c|c|c|c|c|c|c|c|}
\hline \multirow{2}{*}{$\begin{array}{l}\text { Tissue } \\
\text { Substitute }\end{array}$} & \multirow[b]{2}{*}{ RED } & \multirow{2}{*}{$\begin{array}{c}\text { Physical } \\
\text { Density }\left[\mathrm{g} / \mathrm{cm}^{3}\right]\end{array}$} & \multicolumn{6}{|c|}{ Elemental Composition by Percentage Weight } \\
\hline & & & $\mathrm{C}$ & $\mathrm{H}$ & $\mathrm{o}$ & $\mathrm{N}$ & $\mathrm{Cl}$ & Other \\
\hline$\overline{\mathrm{MS} 11^{29}}$ & 0.96 & 0.99 & 67.97 & 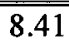 & 18.87 & 2.27 & 0.13 & $\mathrm{Cu}(2.35)$ \\
\hline Hard Bone $\mathrm{e}^{29}$ & 1.71 & 1.84 & 31.36 & 3.10 & 37.57 & 0.99 & 0.05 & $\mathrm{Ca}(2.52)$ \\
\hline $\mathrm{Air}^{18}$ & 0.001 & 0.001 & 0.012 & & 23.18 & 75.53 & & $\operatorname{Ar}(1.28)$ \\
\hline Lung $^{30}$ & 0.28 & 0.288 & 60.50 & 8.38 & 17.28 & 1.68 & 0.15 & $\begin{array}{r}\mathrm{Mg}(11.17) \\
\mathrm{Si}(0.84))\end{array}$ \\
\hline
\end{tabular}

The configuration of the phantoms is described in detail below:

\section{Bone Slab Phantom}

The phantom was composed of two slabs of bone equivalent material, each 0.5 $\mathrm{cm}$ thick, positioned below a $1.0 \mathrm{~cm}$ thick slab of MS11, and on top of the backscatter assembly. A graphical representation of this phantom is shown in Figure 12 (a).

\section{Lung Slab Phantom}

The phantom was formed of two slabs of lung equivalent material, each $1.0 \mathrm{~cm}$ thick. These slabs were positioned below a $1.0 \mathrm{~cm}$ thick slab of MS11, and on top of the backscatter assembly. This phantom is shown in Figure 12 (b). 


\section{Bone Cylinder Phantom}

The bone cylinder phantom consisted of a $1.2 \mathrm{~cm}$ thick slab of MS11with a hard bone cylinder insert positioned at the center. The face of the cylinder, $1.0 \mathrm{~cm}$ in

diameter, was flush with the bottom surface of the slab. The bone inhomogeneity extended $1.0 \mathrm{~cm}$ into the slab, with $0.2 \mathrm{~cm}$ of MS11 above it. This slab was located (face down) below a $1.0 \mathrm{~cm}$ thick slab of MS11, and on top of the backscatter assembly. This phantom is shown in Figure 12 (c).

\section{Air Cylinder Phantom}

A $2.0 \mathrm{~cm}$ thick slab of MS11 with a cylindrical hole was used to form the air cylinder phantom. The air cylinder, located at the center of the slab, was parallel to the beam axis. The air cylinder had a $1.0 \mathrm{~cm}$ diameter and extended $1.8 \mathrm{~cm}$ into the slab, with $0.2 \mathrm{~cm}$ of MS11 above it. This slab was placed below a $1.0 \mathrm{~cm}$ thick slab of MS11, and on top of the backscatter assembly. This phantom is shown in Figure 12 (d).

\section{Trachea and Spine Phantom}

The trachea and spine phantom was composed of a $15.0 \times 15.0 \times 4.7 \mathrm{~cm}^{3} \mathrm{slab}$ with two different inhomogeneities. Located $0.2 \mathrm{~cm}$ below the slab surface was a cylindrical hole, $2.6 \mathrm{~cm}$ in diameter, which extends across the width of the phantom. The cylindrical hole (an example of a 2D inhomogeneity) was perpendicular to the beam axis and was representative of the trachea. Along the bottom of the phantom $(0.5 \mathrm{~cm}$ below the cylindrical hole, and flush with the bottom of the slab) were 4 hard bone cylindrical discs placed $0.5 \mathrm{~cm}$ edge to edge. The discs, $2.5 \mathrm{~cm}$ in diameter and $1.4 \mathrm{~cm}$ thick were parallel with the beam axis. 
These discs represented the spine. This slab was positioned below a $1.0 \mathrm{~cm}$ thick slab of MS11, and above the backscatter assembly. This phantom is shown in Figure 12 (e).

(a) ERB Bone Slab Phantom

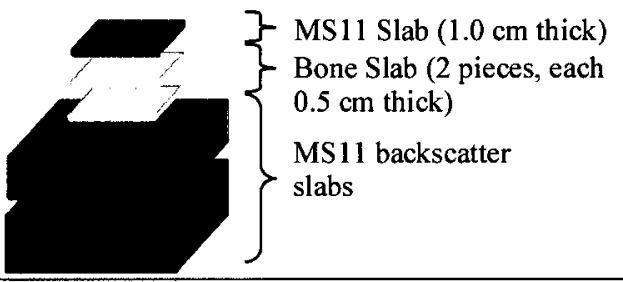

(c) ERB Bone Cylinder Phantom

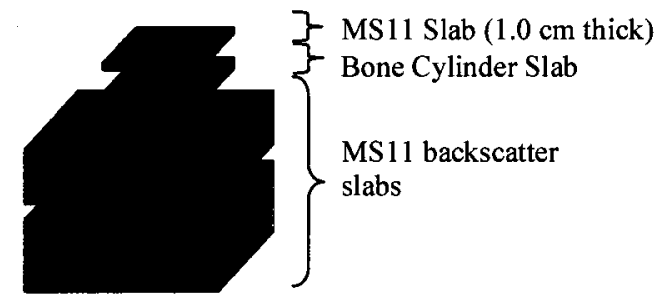

(b) ERB Lung Slab Phantom

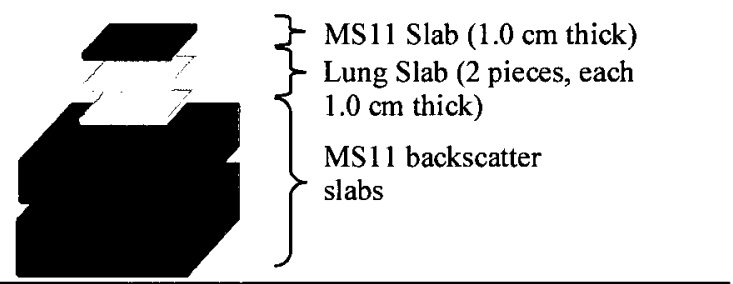

Bone Cylinder Slab

Bottom View, face up orientation

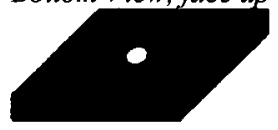

Central Slice Profile, face down orientation D

(d) ERB Air Cylinder Phantom

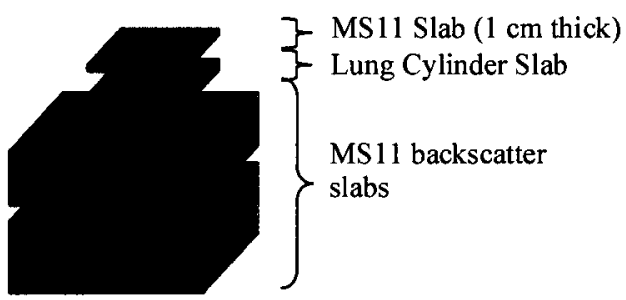

Air Cylinder Slab

Bottom View, face up orientation
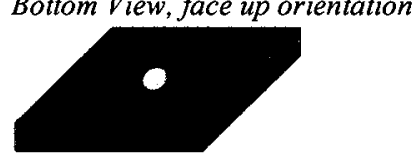

Central Slice Profile, face down orientation

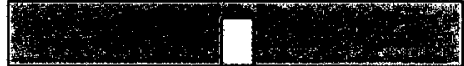

(e) ERB Trachea and Spine Phantom

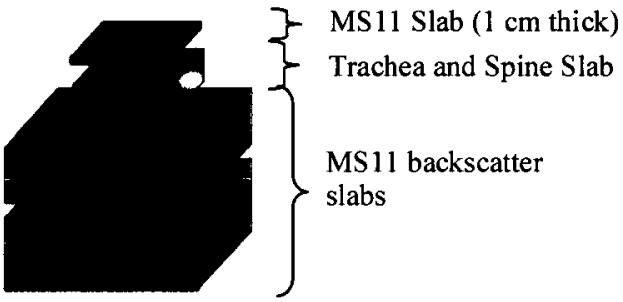

Trachea and Spine Slab Bottom View

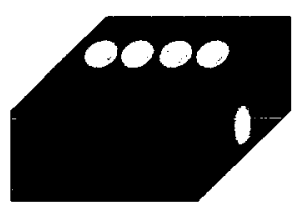

Central Transverse and Axial Slice Profiles

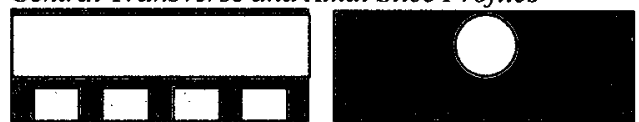

Figure 12. The different configurations of the epoxy resin based phantoms used for $D_{m}$ and $D_{w}$ calculations. 
Vector-based and CT image-based plans were created for each of the epoxy resin-based phantoms. Each plan was designed to deliver $200 \mathrm{MU}$ using 9 and $17 \mathrm{MeV}$ beams, where $200 \mathrm{MU}$ corresponds to $200 \mathrm{cGy}$ at $\mathrm{d}_{\max }$ in a homogeneous water phantom. Each plan was calculated twice, once using the $D_{m}$ method and again using the $D_{w}$ method. This method allows for the direct comparison of several calculations and measurements for two different energies:

- $\mathrm{D}_{\mathrm{w}}$ vs. $\mathrm{D}_{\mathrm{m}}$ calculations for well-defined vector-based anatomies

- $D_{w}$ vs $D_{m}$ for image-based anatomies, whose resolution is limited to that of the image itself and subject to some artifacts

- Comparison between $D_{w}$ calculations for the vector and the image based anatomies

- Comparison between $D_{m}$ calculations for the vector and the image based anatomies

- Comparison of calculations with measurements of the film experiments

The treatment plans were delivered to the phantoms, and using GafChromic EBT1 film, the dose at different depths was measured. The purpose was to evaluate the agreement between calculated and experimental data.

The comparison between the $D_{w}$ and $D_{m}$ calculations was to show the differences that exist for inhomogeneities in a variety of geometrical configurations and determine whether they are larger for vector or image-based anatomies. Although we realized that patient anatomies are rarely represented using a vector-based plan, it should help define why certain dose characteristics were visible in image-based anatomies that were not visible in vector-based anatomies or vice versa. 


\subsection{Calculation of treatment plans for epoxy resin vector-based phantoms}

Vector-based anatomies, with bulk densities and well-defined boundaries, were created for each phantom configuration. The bulk RED values, see Table 2, assigned to each inhomogeneity, with the exception of air, were based on the values presented by White $e t$ $a l^{29,30}$ The RED for air cavities ${ }^{18}$ within the phantoms was 0.001 . These vector-based phantoms were then exported into the External Beam Planning module where the 9 and $17 \mathrm{MeV}$ plans were generated for each of them. Other than the beam energy, both plans were identical. The beams, perpendicularly incident on the center of the phantom surface, were modeled to have a $10 \times 10 \mathrm{~cm}^{2}$ field size and an SSD $=100 \mathrm{~cm}$. The plan specifications are listed in Table 3.

Table 3 Treatment plan parameters used to create the vector and image based treatment plans for the epoxy resin phantoms.

\begin{tabular}{|l|l|}
\hline Parameter \\
\hline \hline Beam Energy & 9 or $17 \mathrm{MeV}$ \\
\hline Field Size & $10 \times 10 \mathrm{~cm}^{2}$ \\
\hline SSD & $100 \mathrm{~cm}$ \\
\hline Display matrix & $0.1 \times 0.1 \mathrm{~cm}^{2}$ \\
\hline Gantry Angle & $0^{0}$ \\
\hline Inhomogeneity correction & $O N$ \\
\hline MU & 200 \\
\hline
\end{tabular}

The display matrix was set to $0.1 \mathrm{~cm}$ in the $\mathrm{x}$ and $\mathrm{z}$ directions of the transverse plane. Using inhomogeneity corrections, the plans were calculated to deliver $200 \mathrm{MU}$, which corresponds to $200 \mathrm{cGy}$ at $\mathrm{d}_{\max }$ in a homogeneous water phantom. Both plans were calculated twice, once using the $D_{m}$ approach, and then again using the $D_{w}$ approach. The image pixel sizes and slice thicknesses are presented in Table 4, along with the display matrix and calculation matrix voxel sizes. 
Table 4 CT-based and vector-based anatomy properties. Variation in voxel size is due to variations in the size of the external dimensions of the phantom.

\begin{tabular}{|c|c|c|c|c|c|c|c|}
\hline & $\begin{array}{c}\text { Pixel Size } \\
(\mathrm{cm})\end{array}$ & \multicolumn{2}{|c|}{ Slice Thickness $(\mathrm{cm})$} & \multicolumn{2}{|c|}{$\begin{array}{c}\text { Display Matrix } \\
(\mathrm{cm})\end{array}$} & \multicolumn{2}{c|}{$\begin{array}{c}\text { Calculation Matrix } \\
\left(\mathrm{X} \times \mathrm{X} \times \mathrm{cm}^{3}\right)\end{array}$} \\
\cline { 2 - 8 } Phantom & CT Image & $\begin{array}{c}\text { Image- } \\
\text { based } \\
\text { anatomy }\end{array}$ & $\begin{array}{c}\text { Vector- } \\
\text { based } \\
\text { anatomy }\end{array}$ & $\begin{array}{c}\text { Image-based } \\
\text { anatomy }\end{array}$ & $\begin{array}{c}\text { Vector } \\
\text { anatomy }\end{array}$ & $\begin{array}{c}\text { Image- } \\
\text { based } \\
\text { anatomy }\end{array}$ & $\begin{array}{c}\text { Vector- } \\
\text { based } \\
\text { anatomy }\end{array}$ \\
\hline $\begin{array}{c}\text { Hard bone } \\
\text { Slab }\end{array}$ & 0.06836 & 0.5 & 0.1 & $0.1 \times 0.5 \times 0.1$ & $0.1 \times 1.0 \times 0.1$ & 0.273 & 0.293 \\
Lung Slab & 0.06836 & 0.5 & 0.1 & $0.1 \times 0.5 \times 0.1$ & $0.1 \times 1.0 \times 0.1$ & 0.273 & 0.293 \\
$\begin{array}{c}\text { Hard bone } \\
\text { Cylinder } \\
\text { Air }\end{array}$ & 0.06836 & 0.2 & 0.1 & $0.1 \times 0.2 \times 0.1$ & $0.1 \times 1.0 \times 0.1$ & 0.273 & 0.293 \\
$\begin{array}{c}\text { Cylinder } \\
\text { Trachea } \\
\text { and Spine }\end{array}$ & 0.06836 & 0.2 & 0.1 & $0.1 \times 0.2 \times 0.1$ & $0.1 \times 1.0 \times 0.1$ & 0.273 & 0.293 \\
\hline
\end{tabular}

The phantoms were calculated with 50,000 electron histories $\mathrm{cm}^{-2}$. Cygler et $a l^{25}$ found an overall uncertainty of less than $1.5 \%$ uncertainty for plans using a $10 \times 10 \mathrm{~cm}^{2}$ field size, 50,000 electron histories $\cdot \mathrm{cm}^{-2}$. These uncertainties were calculated for plans with voxel sizes between $0.3 \times 0.3 \times 0.3 \mathrm{~cm}^{3}$ and $0.49 \times 0.49 \times 0.49 \mathrm{~cm}^{3}$. The uncertainty of the calculations presented are less than $1 \%$ for doses larger than $50 \%$ of $\mathrm{D}_{\max }$.

The axial and lateral dose profile calculations for each vector and image-based phantom are presented in the figures located in their respective sections. The axial and lateral dose profiles have been normalized to $D_{\max }$ in a homogeneous water phantom, 200 cGy. Included in these figures are the DVHs of the heterogeneities in the phantom and a dose difference map depicting the absolute differences between $D_{m}$ and $D_{w}$. The dose difference map shows a difference rather than a ratio or percent difference of dose so that the geometry of the phantoms is still clearly visible. This allows the reader to visualize 
where the differences occur with respect to the inhomogeneities in the phantom. The lateral dose profiles presented were taken at the depth of maximum difference between $D_{m}$ and $D_{w}$ calculations found along the axial dose profile.

\subsubsection{Results: Hard bone slab epoxy resin vector-based phantom}

For the axial dose profiles of the $9 \mathrm{MeV}$ bone slab phantom, Figure 13, $\mathrm{D}_{\mathrm{w}}$ is larger than $\mathrm{D}_{\mathrm{m}}$ by up to $12 \%$ (26.7 cGy) within the bone. This difference occurred at a depth of 1.6 $\mathrm{cm}$ from the surface, near the center of the bone. The lateral dose profiles, taken at 1.6 $\mathrm{cm}$, also differ on average by $12 \%$ (24 cGy) across the width of the field. The dose profile is consistently larger when calculated to $D_{w}$, which explains why in the DVHs the dose for volumes below $40 \%$ is larger for $D_{w}$. It is expected for slab volumes where $D_{m}$ and $D_{w}$ are different, that the DVH will deviate for volume smaller than $44 \%$ of the total slab volume, since only roughly $44 \%$ of the slab is within the $10 \times 10 \mathrm{~cm}^{2}$ field of the beam. 

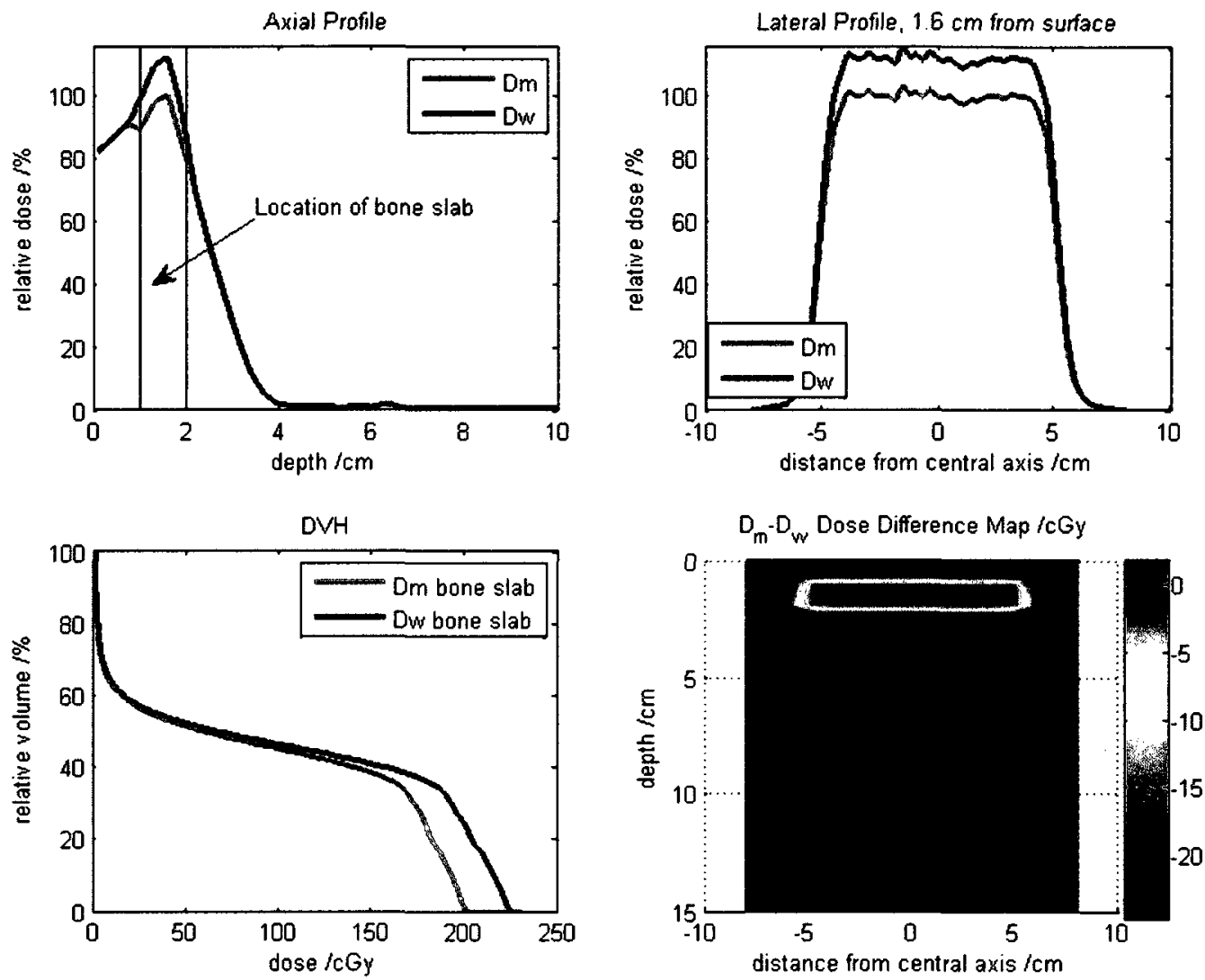

Figure 13. Axial and lateral dose profiles, DVH and dose difference map for the vectorbased hard bone slab phantom, calculated to deliver $200 \mathrm{MU}$ with a $9 \mathrm{MeV}$ beam. Profiles were normalized to $D_{\max }$ in a homogenous water phantom.

Similar results were seen for the $17 \mathrm{MeV}$ bone slab phantom, where the largest difference on the axial dose profile occurs at the deepest edge of the bone slab, $1.9 \mathrm{~cm}$ from the phantom surface. The largest difference along the axial dose profile between $D_{w}$ and $D_{m}$ is $10 \%$ (20 cGy). As in the $9 \mathrm{MeV}$ calculations, the dose-to-water was higher than doseto-medium. 

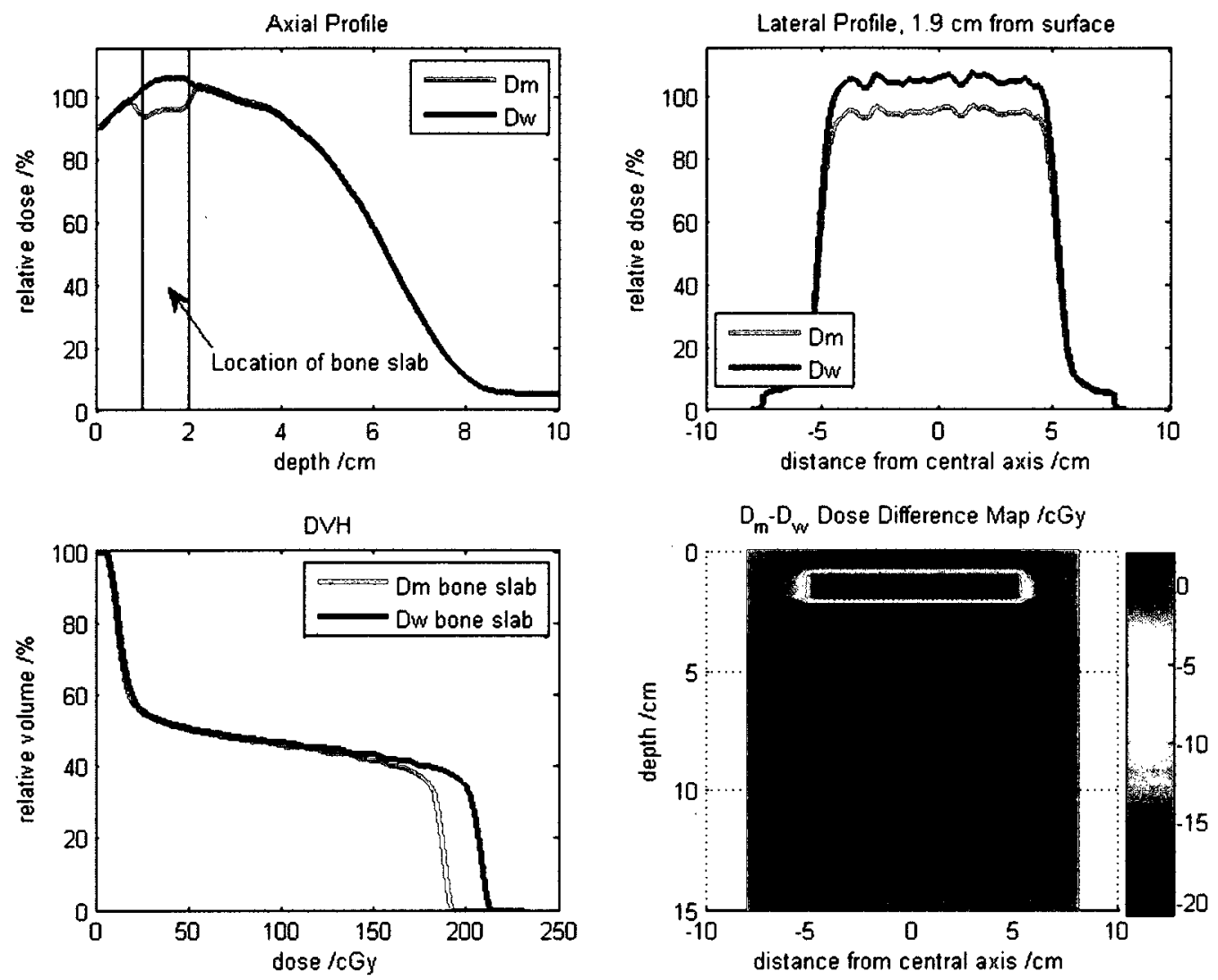

Figure 14. Axial and lateral dose profiles, DVH and dose difference map for the vectorbased hard bone slab phantom, calculated to deliver $200 \mathrm{MU}$ with a $17 \mathrm{MeV}$ beam. Profiles were normalized to $D_{\max }$ in a homogenous water phantom.

The $17 \mathrm{MeV}$ axial dose profiles differ from the $9 \mathrm{MeV}$ profiles in that there is a relatively constant difference between $D_{w}$ and $D_{m}$ axial dose profiles within the bone slab. The lateral dose profiles, taken $1.9 \mathrm{~cm}$ from the surface, disagree on average by $10 \%$ across the width of the bone within the field. The dose volume histogram shows average difference of $11 \%$ for volumes below $34 \%$. The constant difference in dose throughout the bone heterogeneity is particularly more noticeable in the dose difference map. The 17 $\mathrm{MeV}$ dose difference map, when compared to the $9 \mathrm{MeV}$ dose difference map, shows a 
more uniform difference between the calculations throughout the bone. This could be due to less scatter, and a wider $d_{\max }$ region associated with higher energy beams.

The water-to-bone unrestricted mass stopping power ratio for energies evaluated in the bone was $12 \%$ and $13 \%$ above unity for the 9 and $17 \mathrm{MeV}$ plans, respectively. These values are in very good agreement with the percent differences in $D_{m}$ and $D_{w}$.

\subsubsection{Results: Lung slab epoxy resin vector-based phantom}

The 9 and $17 \mathrm{MeV}$ lung slab phantom $\mathrm{D}_{\mathrm{m}}$ and $\mathrm{D}_{\mathrm{w}}$ calculations, Figure 15 and Figure 16, are very similar. 

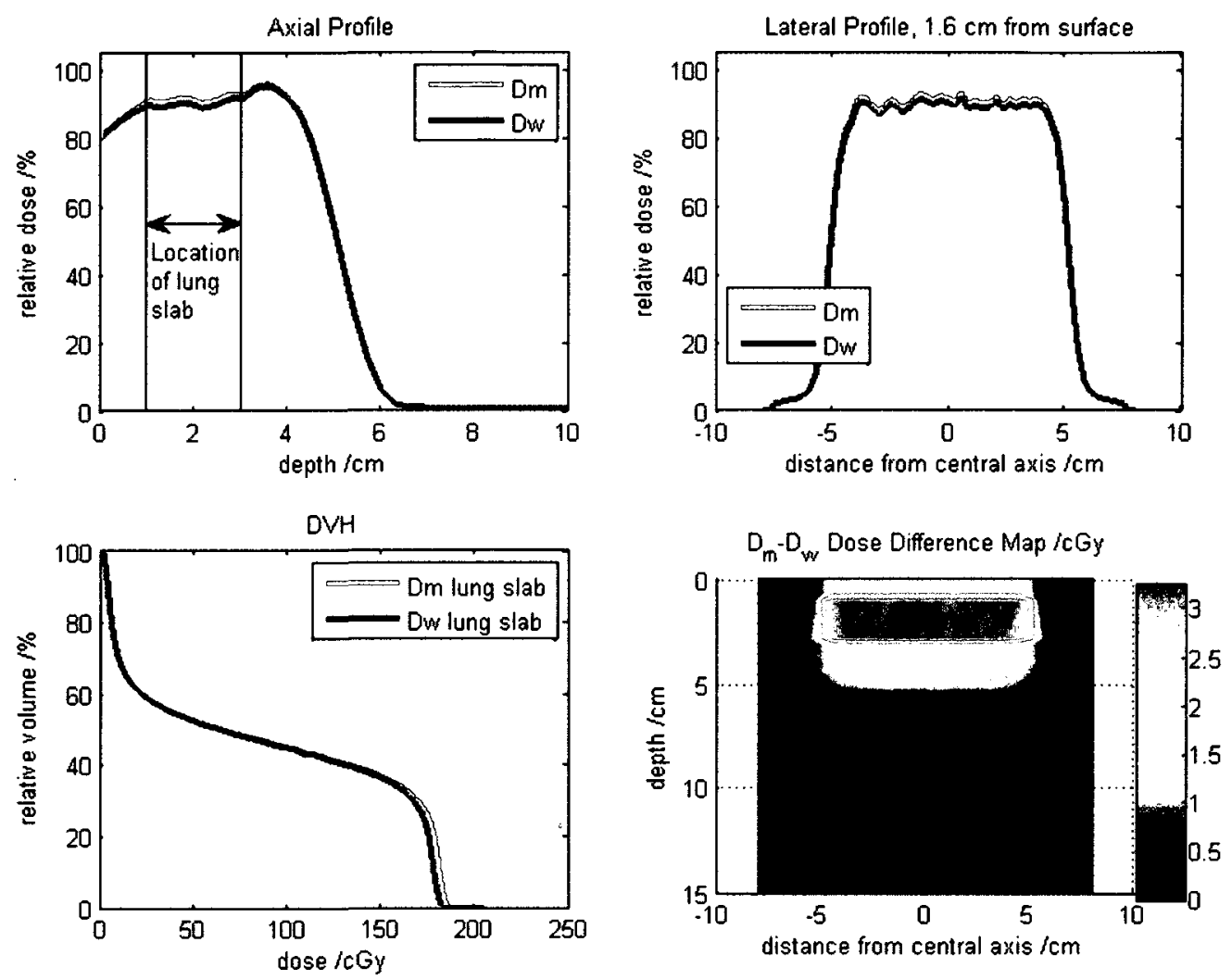

Figure 15. Axial and lateral dose profiles, DVH and dose difference map for the vectorbased lung slab phantom, calculated to deliver $200 \mathrm{MU}$ with a $9 \mathrm{MeV}$ beam. Profiles were normalized to $D_{\max }$ in a homogenous water phantom.

The largest difference between the $9 \mathrm{MeV} \mathrm{D}_{\mathrm{m}}$ and $\mathrm{D}_{\mathrm{w}}$ calculations along the axial dose profile is less than $2 \%$ of the maximum dose. Dose-to-medium is consistently larger than dose-to-water within the lung inhomogeneity. This maximum difference occurred at a depth of $1.6 \mathrm{~cm}$ from the surface, near the center of the lung slab. The lateral dose profiles, taken at $1.6 \mathrm{~cm}$, show similar differences between $D_{w}$ and $D_{m}$, ranging from $1.5 \%$ to $1.6 \%(\sim 3.0 \mathrm{cGy})$. The similarity between the two calculation approaches can be seen in the DVH and dose difference map. The dose difference map shows the largest differences in the lung slab, but these differences do not exceed $1.8 \%$. 

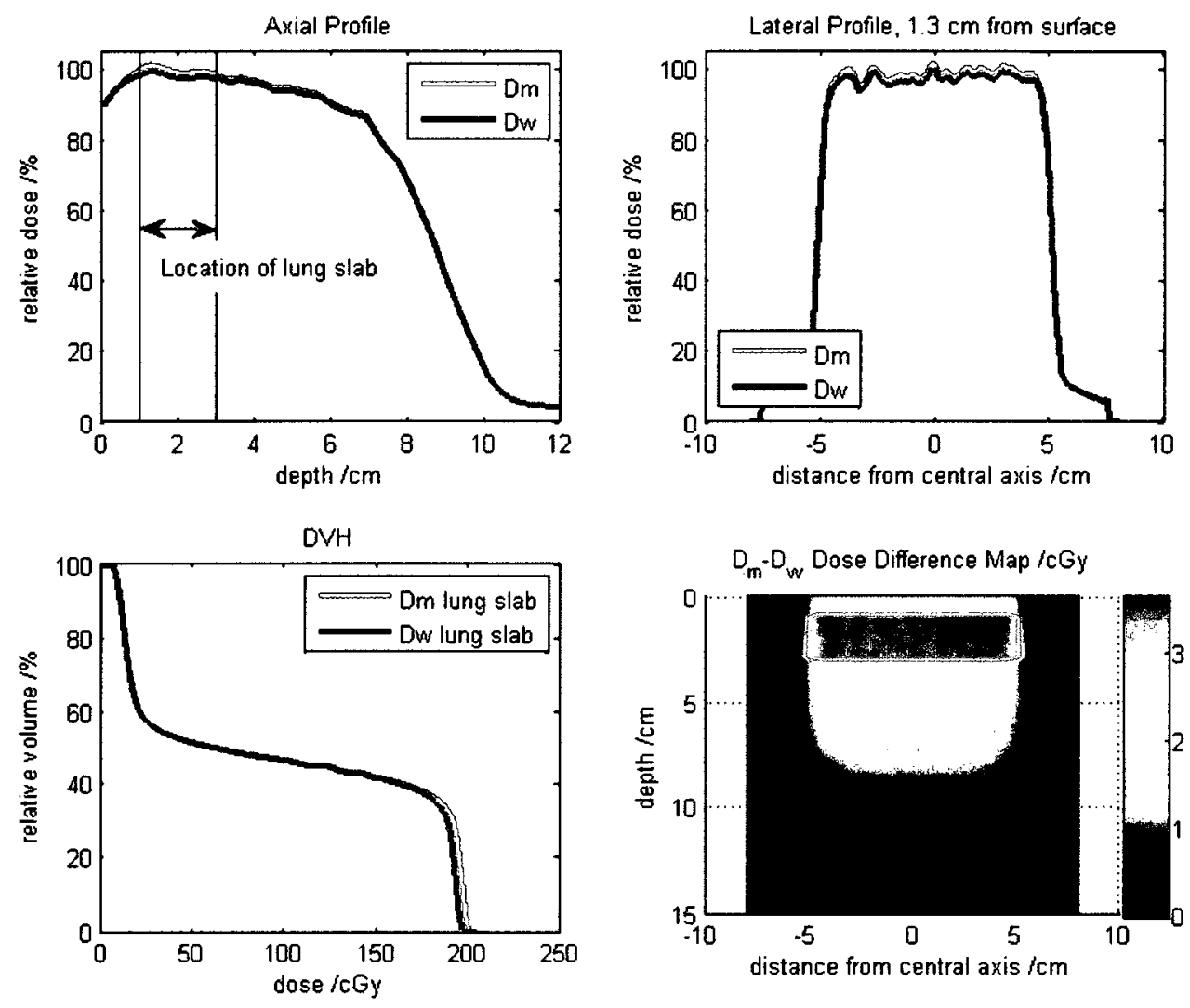

Figure 16. Axial and lateral dose profiles, DVH and dose difference map for the vectorbased lung slab phantom, calculated to deliver $200 \mathrm{MU}$ with a $17 \mathrm{MeV}$ beam. Profiles were normalized to $D_{\max }$ in a homogenous water phantom.

For the $17 \mathrm{MeV}$ lung phantoms, the differences between the doses calculated within the lung slab are only marginally larger than that for the $9 \mathrm{MeV}$ phantom calculations. For the $17 \mathrm{MeV}$ lung slab phantom, the largest difference between the two calculation approaches along the axial dose profile is $1.8 \%$ (3.6 cGy), and it occurs at a depth of 1.3 $\mathrm{cm}$ from the phantom's surface. Dose-to-medium was again larger than dose-to-water calculations. The lateral dose profiles at $1.3 \mathrm{~cm}$ show comparable differences of $1.8 \%$ 
across the width of the field. The differences between the lung slab DVHs are comparable to that of the $9 \mathrm{MeV}$ plans.

The differences seen between the $D_{m}$ and $D_{w}$ calculations agreed well with the water-tolung unrestricted mass collisional stopping powers within the range of energies used in the clinic, with a ratio of approximately 0.985 . This ratio suggests that the $D_{w}$ would be lower than $D_{m}$, which in this case it is.

\subsubsection{Results: Hard bone cylinder epoxy resin vector-based phantom}

Figure 17 and Figure 19 are the 9 and $17 \mathrm{MeV}$ profiles for the hard bone cylinder phantoms in the bone-face-down orientation. The bone is located $1.2 \mathrm{~cm}$ from the surface, and extends to $2.2 \mathrm{~cm}$ into the phantom. 

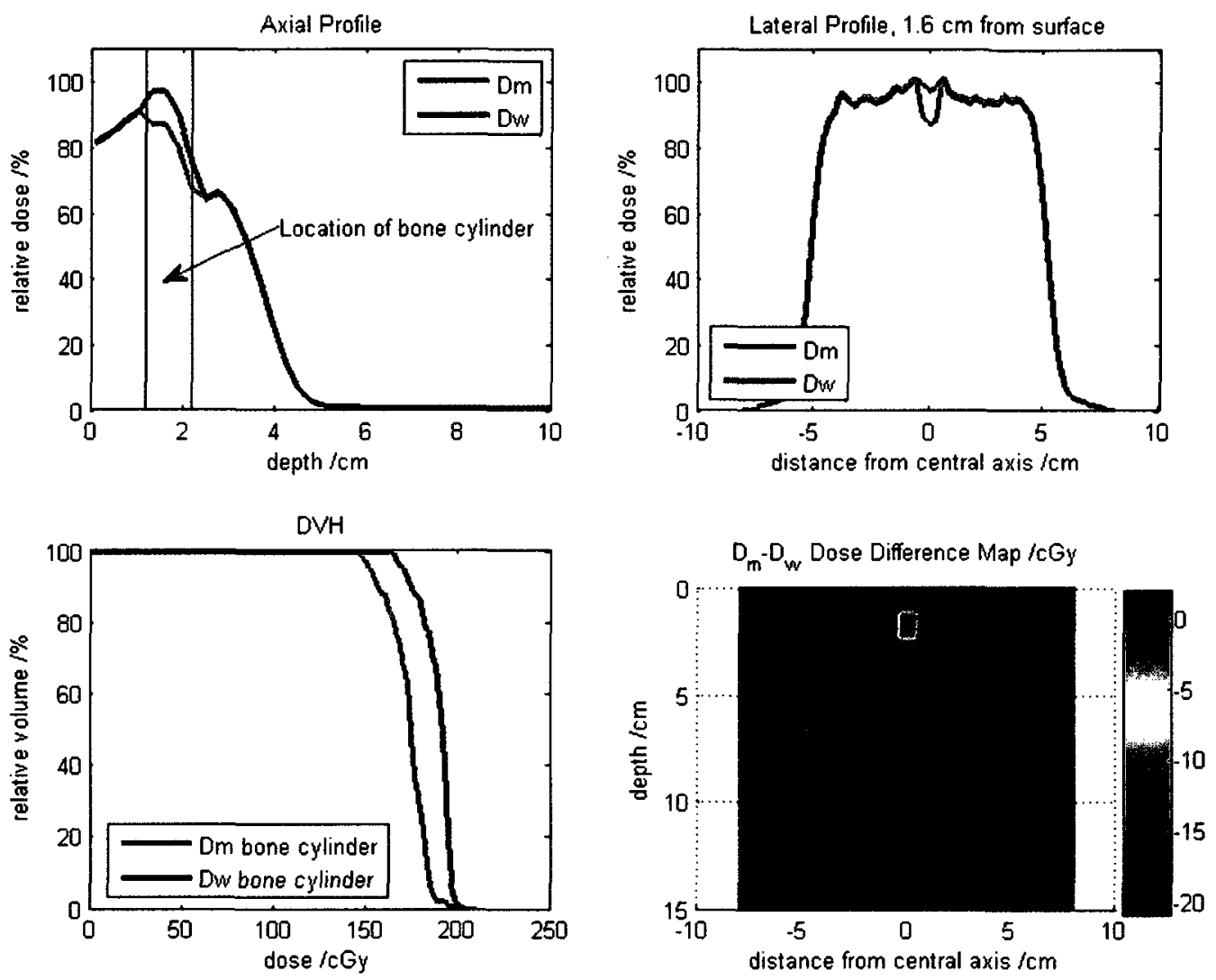

Figure 17. Axial and lateral dose profiles, DVH and dose difference map for the vectorbased hard bone cylinder phantom (bone $1.2 \mathrm{~cm}$ from the surface), calculated to deliver $200 \mathrm{MU}$ with a $9 \mathrm{MeV}$ beam. Profiles were normalized to $D_{\max }$ in a homogenous water phantom.

The axial dose profiles of the $9 \mathrm{MeV}$ hard bone cylinder phantom differ most within the cylinder. The greatest difference is $10 \%$ (20.5 cGy) of the maximum dose at $1.6 \mathrm{~cm}$ from the phantom surface (or $0.4 \mathrm{~cm}$ into the cylinder). Differences of this magnitude only exist for display matrix pixels that lie within calculation matrix pixels that contain exclusively densities of the bone. The location of the display matrix with respect to the calculation matrix is presented in Figure 18. The black grid represents the calculation matrix, the green grid is the display matrix and the blue square is the location of the bone cylinder with respect to the grids. 


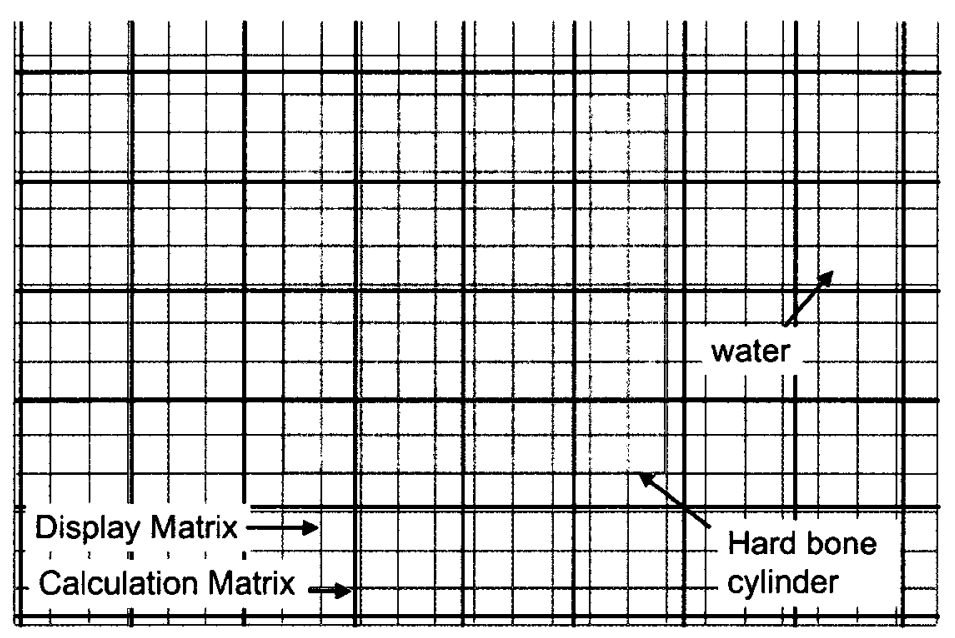

Figure 18. Display matrix (green grid) superimposed on the calculation matrix (black grid) and the hard bone cylinder phantom.

Profiles through the phantoms are more complex due to the location of the inhomogeneity, the calculation voxels, and the display voxels. It is possible that a profile taken near (but not within) an inhomogeneity uses the calculation points that lie within a calculation voxel with properties of the inhomogeneity. It is difficult to know whether these calculations are affected by the voxel assignment.

In the 9 and $17 \mathrm{MeV}$ axial dose profiles, differences between the $D_{m}$ and $D_{w}$ calculations extended beyond the boundaries of the bone cylinder denoted as vertical black lines in Figure 17 and Figure 19. This could partially be attributed to the material properties assigned to the calculation voxels containing the upstream and down stream edges of the bone cylinder. It could also be credited to the difference in scatter. As one would expect, there is little difference between the $D_{m}$ and $D_{w}$ calculation points that lie within the calculation matrix voxels that solely contain water. For the $9 \mathrm{MeV}$ lateral dose profiles, taken $1.6 \mathrm{~cm}$ from the surface, the largest difference between $D_{m}$ and $D_{w}$ is $10 \%(21 \mathrm{cGy})$ at $-0.3 \mathrm{~cm}$ from the beam central axis and within the bone. This shift is roughly the size 
of one calculation matrix voxel. This point could be the cylinder center as seen by the calculation matrix. The DVHs for the bone heterogeneity differ by approximately $10 \%$. This is consistent with the mass collisional stopping power ratio of water to bone for energies within the clinical range.

The differences seen in the calculations for the $17 \mathrm{MeV}$ bone cylinder phantom are similar to those for the $9 \mathrm{MeV}$ phantoms. The calculation and display matrices of the 9 $\mathrm{MeV}$ plan are the same for the $17 \mathrm{MeV}$ plan. Along the bone section of the axial dose profile there is a $9.6 \%(19.2 \mathrm{cGy})$ difference between $D_{m}$ and $D_{w}$. This difference is located at a depth of $1.4 \mathrm{~cm}$ from the surface. The $D_{w}$ lateral dose profiles do not show the existence of the bone heterogeneity within the field. On the other hand, the $D_{m}$ calculation is about $9 \%(19 \mathrm{cGy})$ lower in the section where there is bone. 

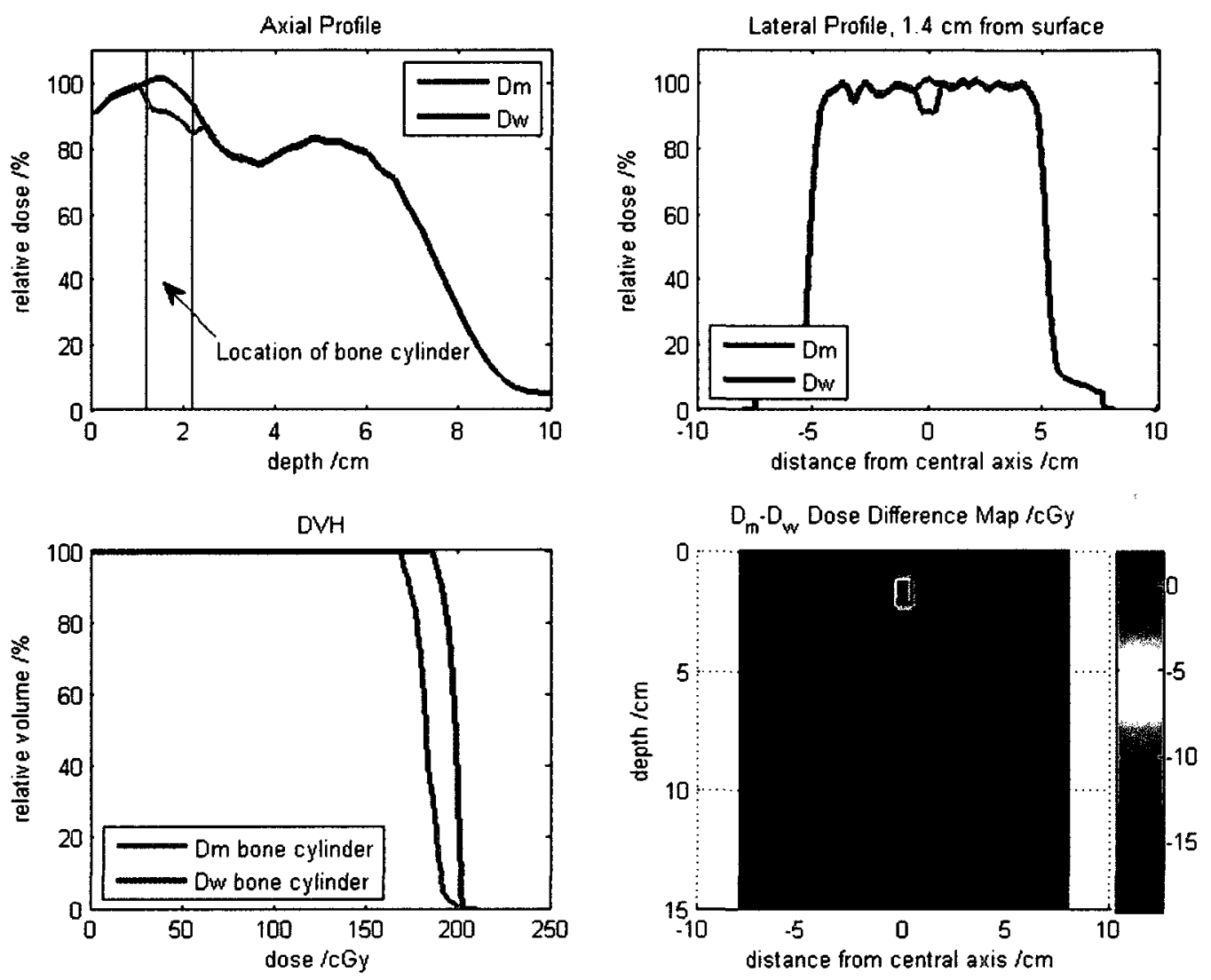

Figure 19. Axial and lateral dose profiles, DVH and dose difference map for the vectorbased hard bone cylinder phantom (bone $1.2 \mathrm{~cm}$ from the surface), calculated to deliver $200 \mathrm{MU}$ with a $17 \mathrm{MeV}$ beam. Profiles were normalized to $D_{\max }$ in a homogenous water phantom.

Similar to the hard bone slab phantoms, the dose within the bone is higher when calculated using the $\mathrm{D}_{\mathrm{w}}$ approach. The differences between the calculation approaches are similar for the respective energies. For both energies, the $D_{w}$ lateral dose profiles (crossing through the bone cylinder) do not show the presence of bone. The differences between $D_{m}$ and $D_{w}$ are consistent with the mass collisional stopping powers within the range of clinical energies, approximately $11 \%$. 


\subsubsection{Results: Air cylinder epoxy resin vector-based phantom}

The profiles, DVH and the dose difference maps are presented in Figure 20 and Figure 21

for the 9 and $17 \mathrm{MeV}$ calculations, respectively. The discrepancy between the $\mathrm{D}_{\mathrm{m}}$ and $D_{\mathrm{w}}$ calculations for the $9 \mathrm{MeV}$ within air cylinder increases with depth. This trend is especially evident in the axial dose profile and the dose difference map presented in Figure 20. This difference grows to $5 \%$ at $2.7 \mathrm{~cm}$ from the surface $(1.5 \mathrm{~cm}$ within the air cylinder). From the lateral dose profile taken at $2.7 \mathrm{~cm}$ from the surface, the $D_{m}$ is larger for the points of the profile that lie around the location of the air cylinder but smaller within the air cavity. The greatest difference between the lateral dose profiles is $5 \%$ at $-0.3 \mathrm{~cm}$ from the central axis. There is a gradual increase in the differences between the $D_{m}$ and $D_{w}$ DVHs of the air cylinder. The percent difference within $100 \%$ of the volume is $1.4 \%$ and increases to $4.2 \%$ at $3.4 \%$ of the volume. 

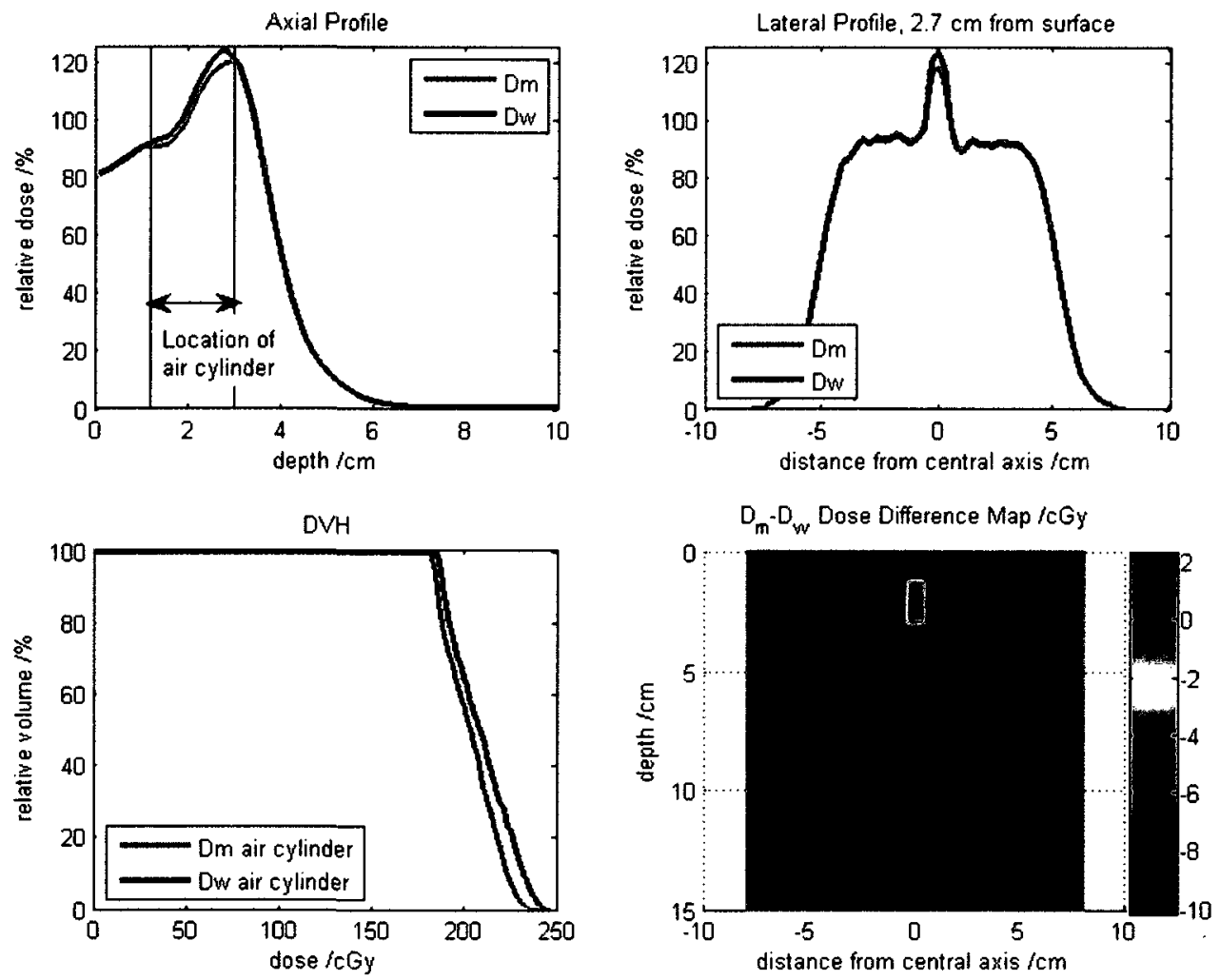

Figure 20. Axial and lateral dose profiles, DVH and dose difference map for the vectorbased air cylinder phantom (cylinder $1.2 \mathrm{~cm}$ from the surface), calculated to deliver 200 $M U$ with a $9 \mathrm{MeV}$ beam. Profiles were normalized to $D_{\max }$ in a homogenous water phantom.

For the axial and lateral dose profiles, the $17 \mathrm{MeV}$ air cylinder phantom calculations around the air cavity do not exhibit any visual differences. However, minor differences are seen in sections of the profiles crossing through the air cavity. The largest difference between $D_{m}$ and $D_{w}$ along the axial dose profile was too small to be appreciated. The largest difference was only $2 \%$ at a depth of $1.4 \mathrm{~cm}$ from the surface $(0.2 \mathrm{~cm}$ into the air cavity). 

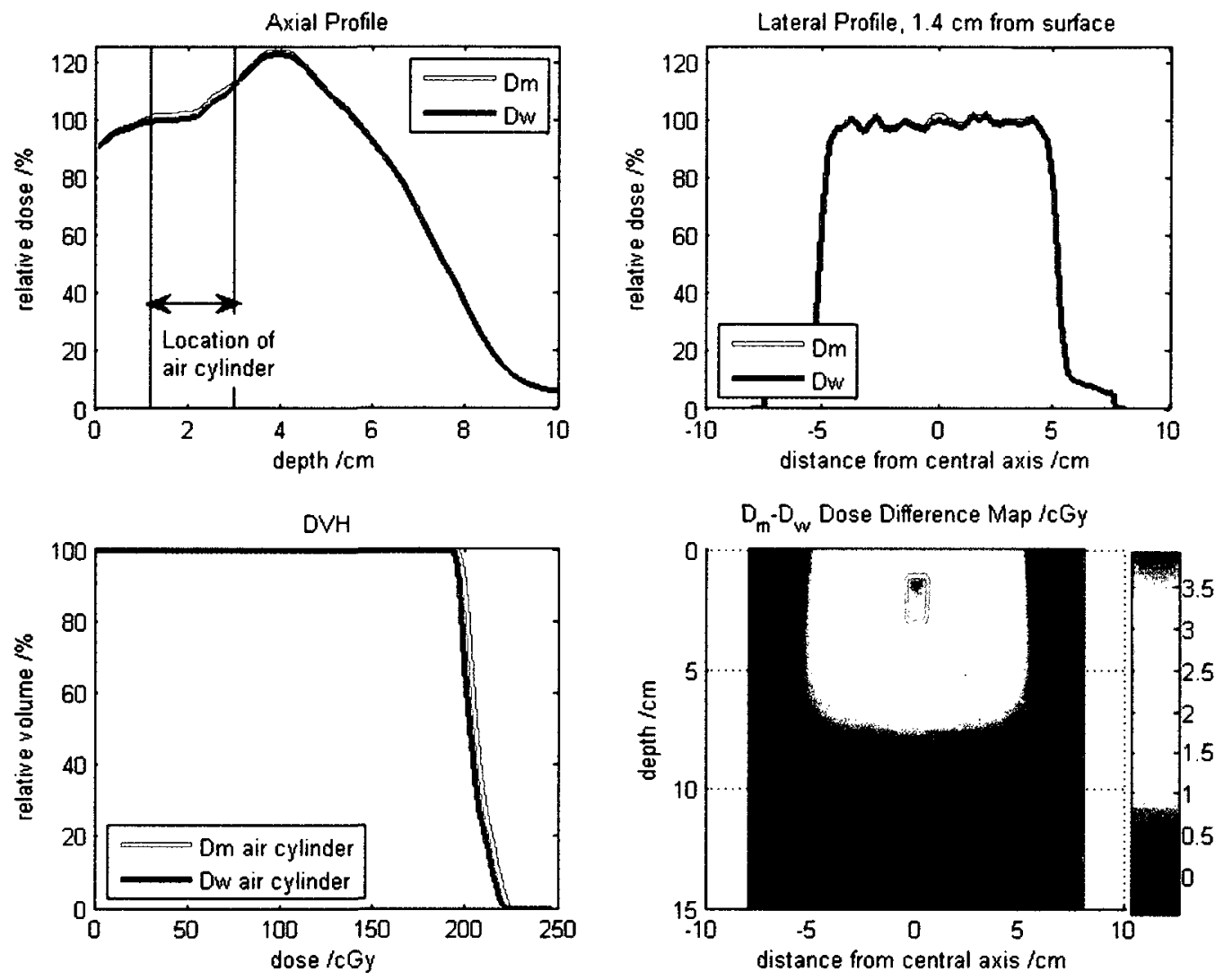

Figure 21. Axial and lateral dose profiles, DVH and dose difference map for the vectorbased air cylinder phantom (cylinder $1.2 \mathrm{~cm}$ from the surface), calculated to deliver 200 $M U$ with a $17 \mathrm{MeV}$ beam. Profiles were normalized to $D_{\max }$ in a homogenous water phantom.

In the lateral dose profiles taken at $1.4 \mathrm{~cm}$ depth, the dose-to-medium calculations are slightly larger than dose-to-water calculations in the location of the air cylinder. The largest difference in the lateral dose profile in the region of the air cavity is approximately $2 \%$ ( $4 \mathrm{cGy}$ ). The $\mathrm{D}_{\mathrm{w}} \mathrm{DVH}$ of the air cavity systematically differs from the $\mathrm{D}_{\mathrm{m}} \mathrm{DVH}$, by approximately $1.6 \%$. 
The mass stopping power ratio of water to air increases rapidly with decreasing energy. Therefore, we expect to see much larger differences between $D_{m}$ and $D_{w}$ calculations for plans using a lower energy.

Figure 22 is the display and calculation matrix superimposed on the central slice of the air cylinder phantom. The surrounding edges of the cylinder lie within calculation voxels that also contain the surrounding medium.

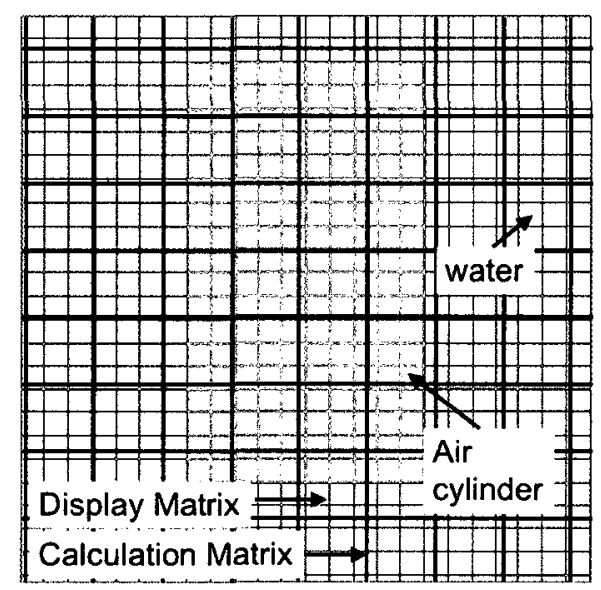

Figure 22. Display matrix (green grid) superimposed on the calculation matrix (black grid) and the air cylinder phantom.

\subsubsection{Results: Trachea and spine epoxy resin vector-based phantom}

The profiles, DVH and the dose difference maps for the trachea and spine phantom calculations are presented in Figure 23 and Figure 24 for the 9 and $17 \mathrm{MeV}$ calculations respectively. Only the DVHs of the air cylinder and the bone centered at $-2.75 \mathrm{~cm}$ from the central axis are displayed. Along the axial dose profile, the largest difference between $D_{m}$ and $D_{w}$ is $3.7 \%$, located $3.6 \mathrm{~cm}$ from the phantom surface, within the air 
cylinder. The $D_{m}$ and $D_{w}$ calculations along the lateral dose profile at this depth differ by roughly the same, $3.8 \%$.
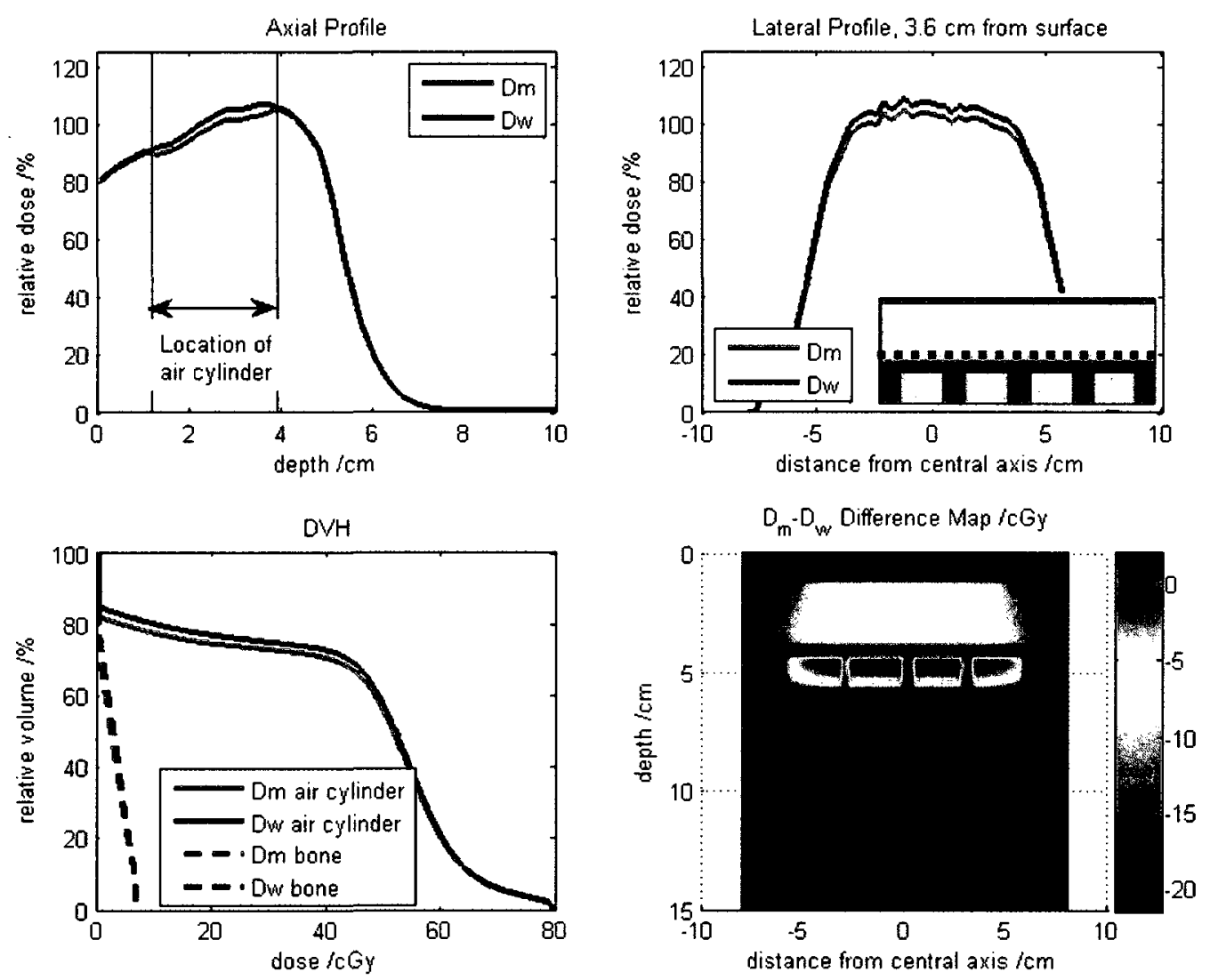

Figure 23. Axial and lateral dose profiles, DVH and dose difference map for the vectorbased trachea and spine phantom, calculated to deliver $200 \mathrm{MU}$ with a $9 \mathrm{MeV}$ beam. The lateral dose profile contains a pictorial description of where the profile was taken within the phantom. Profiles were normalized to $D_{\max }$ in a homogenous water phantom.

There are smaller differences between the $D_{m}$ and $D_{w}$ axial dose calculations for the 17 $\mathrm{MeV}$ profiles than those for the $9 \mathrm{MeV}$ profiles. The maximum difference between the $17 \mathrm{MeV}$ profiles is $2.0 \%$ (3.9 cGy) at a depth of $1.4 \mathrm{~cm}$. 

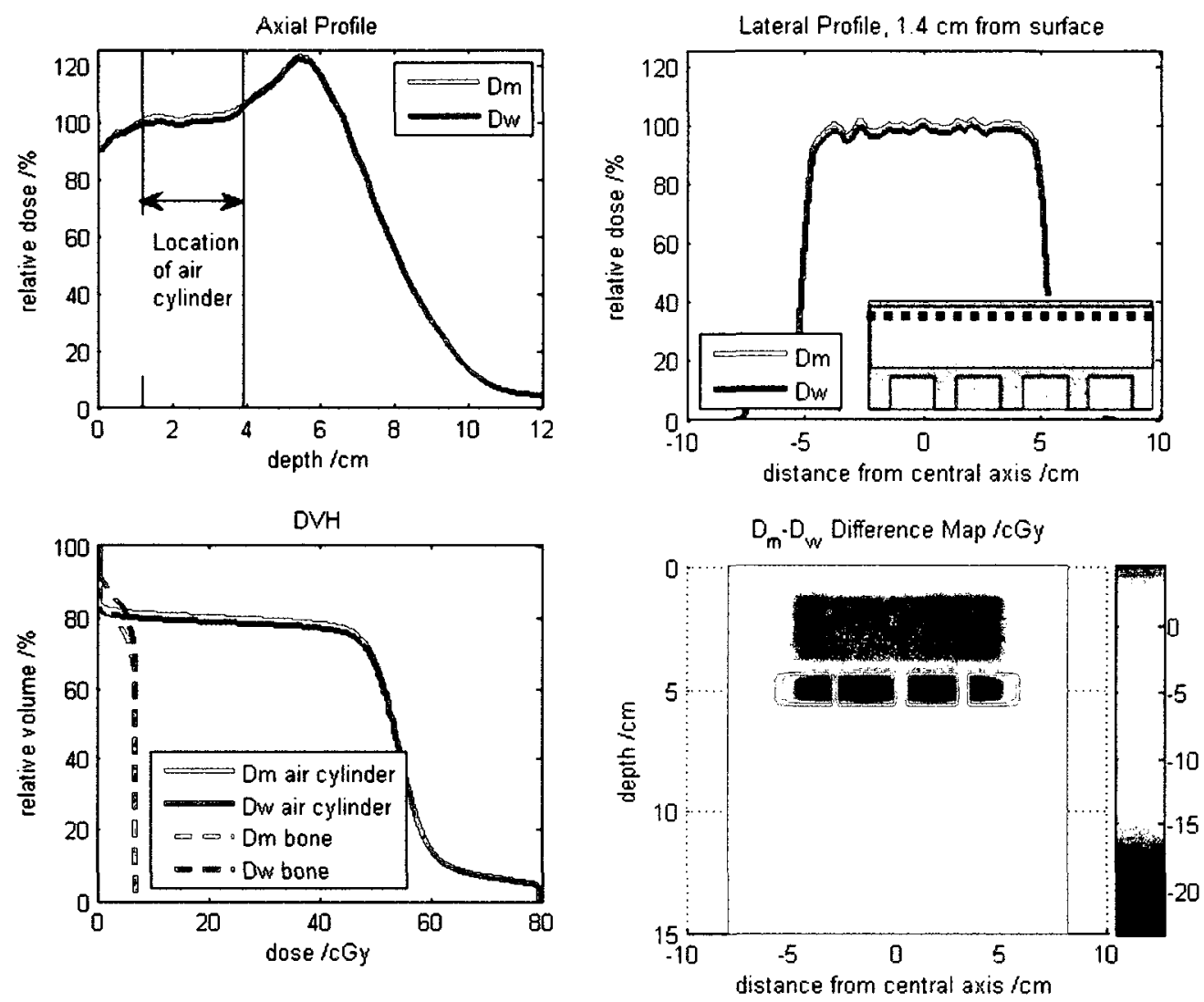

Figure 24. Axial and lateral dose profiles, DVH and dose difference map for the vectorbased trachea and spine phantom, calculated to deliver $200 \mathrm{MU}$ with a $17 \mathrm{MeV}$ beam. The lateral dose profile contains a pictorial description of where the profile was taken within the phantom. Profiles were normalized to $D_{\max }$ in a homogenous water phantom.

When the dose difference maps for the 9 and $17 \mathrm{MeV}$ maps are compared, it was seen that $D_{m}$ was higher than $D_{w}$ in the low-density volumes and smaller than $D_{w}$ in the highdensity volumes. This follows what we expect to see, based on the results for the bone slab and lung cylinder phantoms. From the dose difference maps, the differences between the $9 \mathrm{MeV} \mathrm{D}_{\mathrm{m}}$ and $\mathrm{D}_{\mathrm{w}}$ calculations, within the bone discs, are greater near the edge closest to the phantom surface, unlike the $17 \mathrm{MeV}$ where the difference is approximately uniform. 
The depth of the lateral profiles are shown on the superposition of the calculation and display matrix over an image of the trachea and spine phantom, shown in Figure 25.

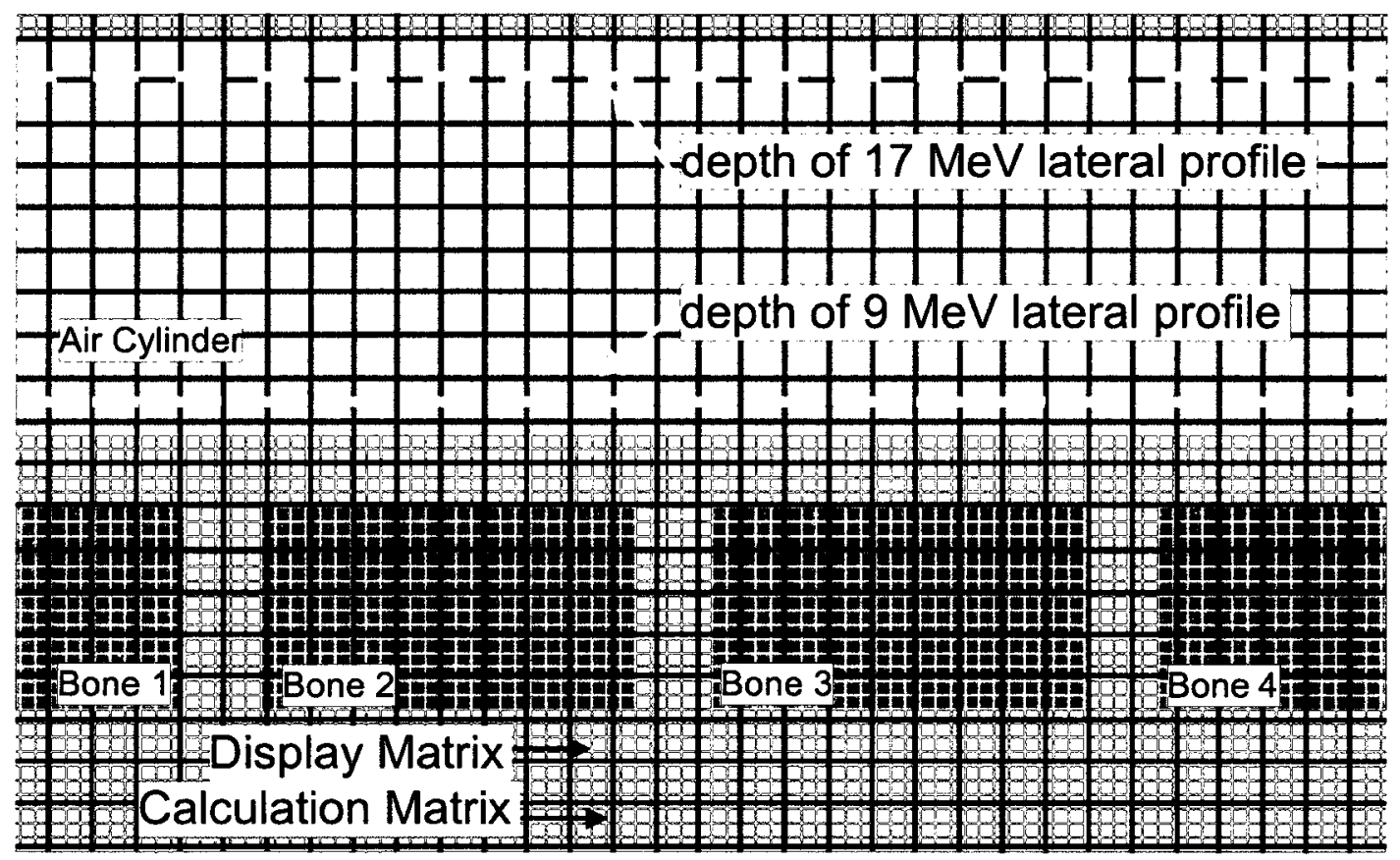

Figure 25. Depth of lateral profiles with respect to the calculation matrix (black grid), display matrix (green matrix), and the inhomogeneities. The 9 and $17 \mathrm{MeV}$ lateral profiles are shown in red and orange respectively. 


\subsection{Calculation of treatment plans for epoxy resin image-based phantoms}

To create an image-based anatomy of the epoxy based phantoms, each phantom was imaged using a Philips Brilliance CT scanner. Phantoms containing slab geometry inhomogeneities were scanned with $0.5 \mathrm{~cm}$ slice thickness. The phantoms containing small cylindrical inhomogeneities and the trachea and spine phantom were scanned with $0.2 \mathrm{~cm}$ slice thicknesses. The images were contoured, and the REDs were assigned automatically based on the $\mathrm{HU}$ value of each individual pixel. The $\mathrm{HU}$ values were converted to REDs using the scanner-specific "BigBore" calibration curve available in the Anatomy Modeling module of Theraplan Plus ${ }^{\circledR}$.

The image-based anatomies of the phantoms were exported into External Beam Planning module. Like the vector-based phantoms, two plans were created for each phantom. The first plan used a $9 \mathrm{MeV}$ beam and the second used a $17 \mathrm{MeV}$ beam. Other than the energy, both plans were identical; the parameters are listed in Table 3 . The beams, perpendicularly incident on the center of the phantom surface, were modeled to have a 10 $x 10 \mathrm{~cm}^{2}$ field size and a $100 \mathrm{~cm}$ SSD. Using inhomogeneity corrections, the plans were calculated to deliver $200 \mathrm{MU}$, which corresponds to $200 \mathrm{cGy}$ at $\mathrm{d}_{\max }$ in a homogeneous water phantom. Both plans were calculated twice, once using the $D_{m}$ approach, and then again using the $D_{w}$ approach.

The axial and lateral dose profiles for the each image-based phantom are presented in the figures located in their respective sections. Included in these figures are the DVHs of the heterogeneity within the phantom and a dose difference map depicting the differences between $D_{m}$ and $D_{w}$. Similarly as for vector anatomies, the dose difference map is 
presented as a difference rather than a ratio or percent difference so that the geometry of the phantoms is still visible in the dose difference map. This allows the reader to visualize where the differences occur with respect to the inhomogeneities in the phantom. The lateral dose profiles presented were taken at the depth of maximum difference between $D_{m}$ and $D_{w}$ calculations along the axial dose profile.

\subsubsection{Results: Hard bone slab epoxy resin image-based phantom}

Figure 26 presents the profiles, the DVH of the bone slab inhomogeneity, and the dose difference map of the $9 \mathrm{MeV}$ hard bone slab image-based phantom calculations. For the 9 $\mathrm{MeV}$ beam, the maximum discrepancy between $D_{m}$ and $D_{w}$ along the axial dose profile for the image-based bone slab phantom is $8.4 \%(16.9 \mathrm{cGy})$ at $1.6 \mathrm{~cm}$ from the phantom surface. The lateral dose profile was taken at the depth of the greatest difference along the axial dose profile, and showed a roughly consistent difference of $8.7 \%$ (17.3 cGy) between the calculation approaches. The DVHs of the bone slab agree rather well for at least $40 \%$ of the volume; beyond that point there is a consistent difference between $D_{m}$

and $D_{w}$. It is expected that the DVH would deviate at this point since only approximately $40 \%$ of the bone is within the $10 \times 10 \mathrm{~cm}^{2}$ field of the beam. 

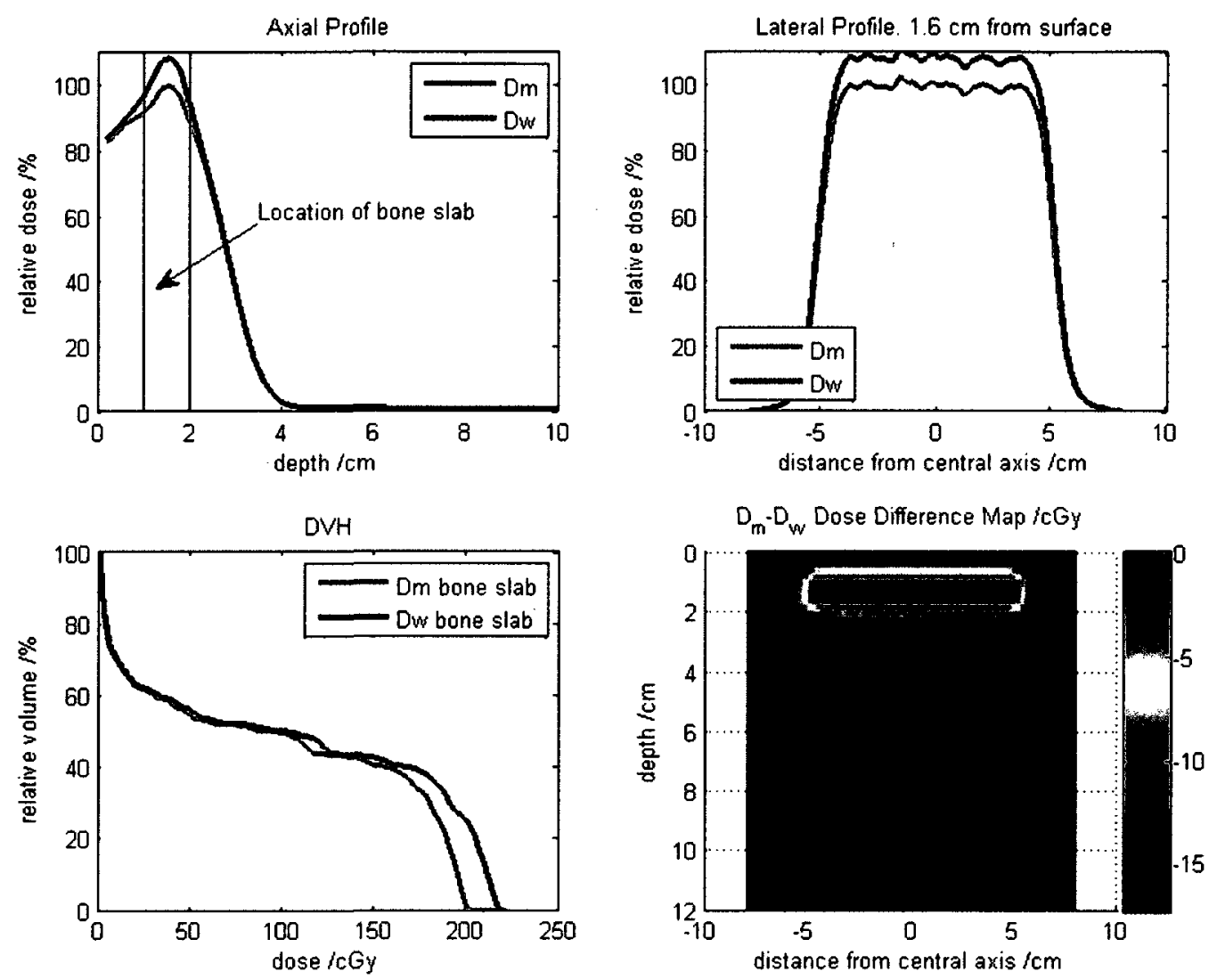

Figure 26. Axial and lateral dose profiles, DVH and dose difference map for the imagebased hard bone slab phantom, calculated to deliver $200 \mathrm{MU}$ with a $9 \mathrm{MeV}$ beam. Profiles were normalized to $D_{\max }$ in a homogenous water phantom.

The dose difference map for the $17 \mathrm{MeV}$ bone slab phantom, Figure 27, shows evidence of having used an assembly of water slabs as backscatter. At a depth of approximately 7 $\mathrm{cm}$, there are ripples in the dose distribution; this is due to a tiny air gap between the MS11 slabs. This gap was clearly visible in the CT images. 

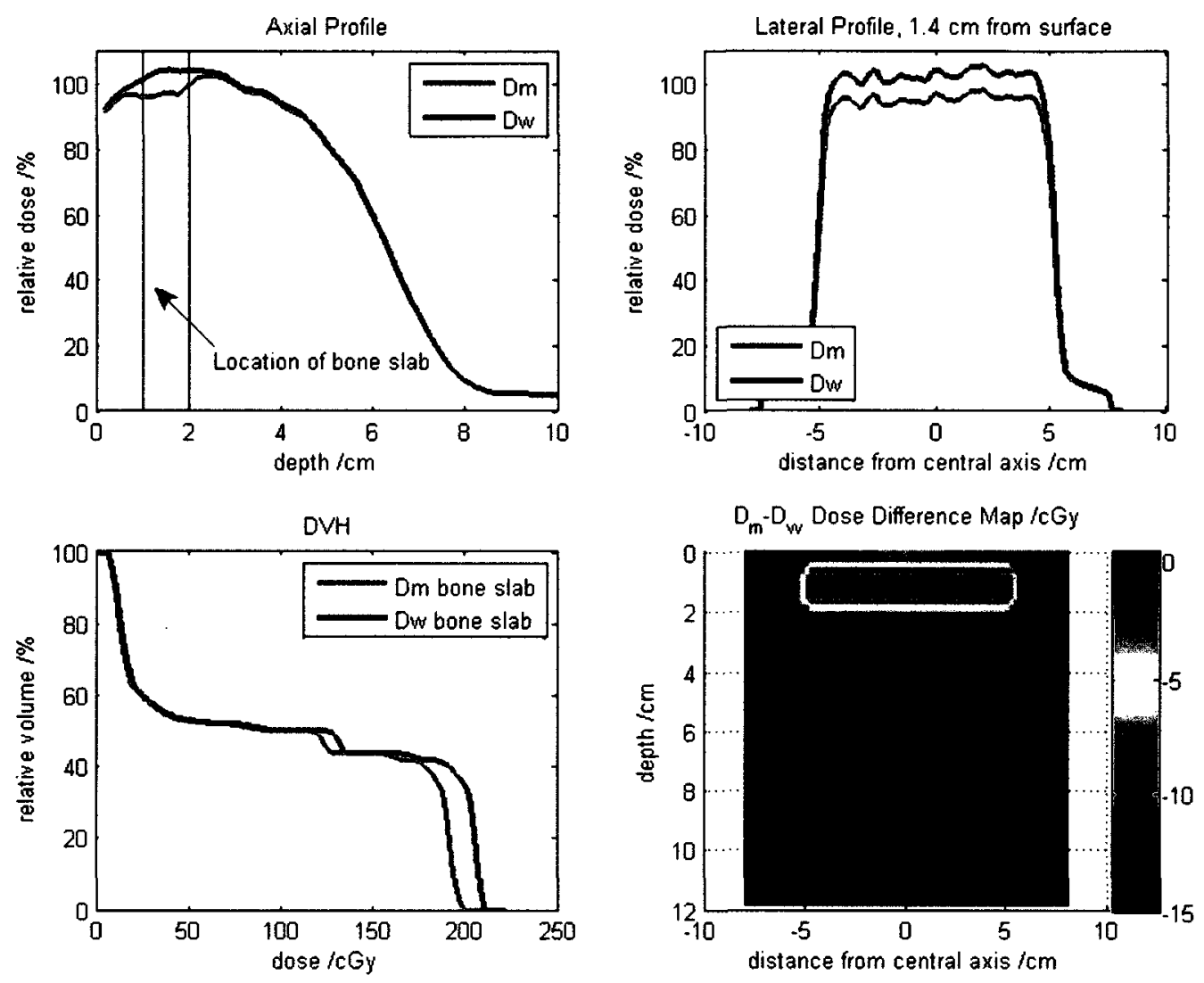

Figure 27. Axial and lateral dose profiles, DVH and dose difference map for the imagebased hard bone slab phantom, calculated to deliver $200 \mathrm{MU}$ with a $17 \mathrm{MeV}$ beam. Profiles were normalized to $D_{\max }$ in a homogenous water phantom.

Similar to the results of the vector based bone slab, the agreement between the $D_{m}$ and $D_{w}$ calculations only slightly improves with energy. The largest difference between $D_{m}$ and $\mathrm{D}_{\mathrm{w}}$ was $7.3 \%(14.7 \mathrm{cGy})$ along the axial dose profile at a depth of $1.4 \mathrm{~cm}$ from the surface. This difference is consistent with the differences between the two calculation approaches along the lateral dose profile taken at $1.4 \mathrm{~cm}$. The dose volume histogram shows a systematic difference between $D_{m}$ and $D_{w}$ for 0 to $40 \%$ of the volume. The differences between the calculations for the image-based phantom are smaller than those for the vector-based phantom; this could be due to the assigned material properties within 
the homogeneity, or differences in voxel size (the image-based anatomy has a smaller voxel size). Another consideration is that the vector-based anatomy was assigned a bulk density, whereas the CT-based phantom has densities assigned pixel by pixel that vary within the heterogeneity. Variations in densities will affect the dose distribution. This is true for all the phantoms presented.

\subsubsection{Results: Lung slab epoxy resin image-based phantom}

The 9 and $17 \mathrm{MeV}$ lung phantom calculations show only small differences between the $D_{m}$ and $D_{w}$ calculations, Figure 28 and Figure 29. The differences between the lateral dose profile calculations for either energy do not exceed $2 \%$ of the maximum lateral dose at their corresponding depths. The lateral dose profiles were taken at $1.2 \mathrm{~cm}$ from the surface for both sets of profiles since this is the depth along the axial dose profile where $D_{m}$ and $D_{w}$ differed most. 

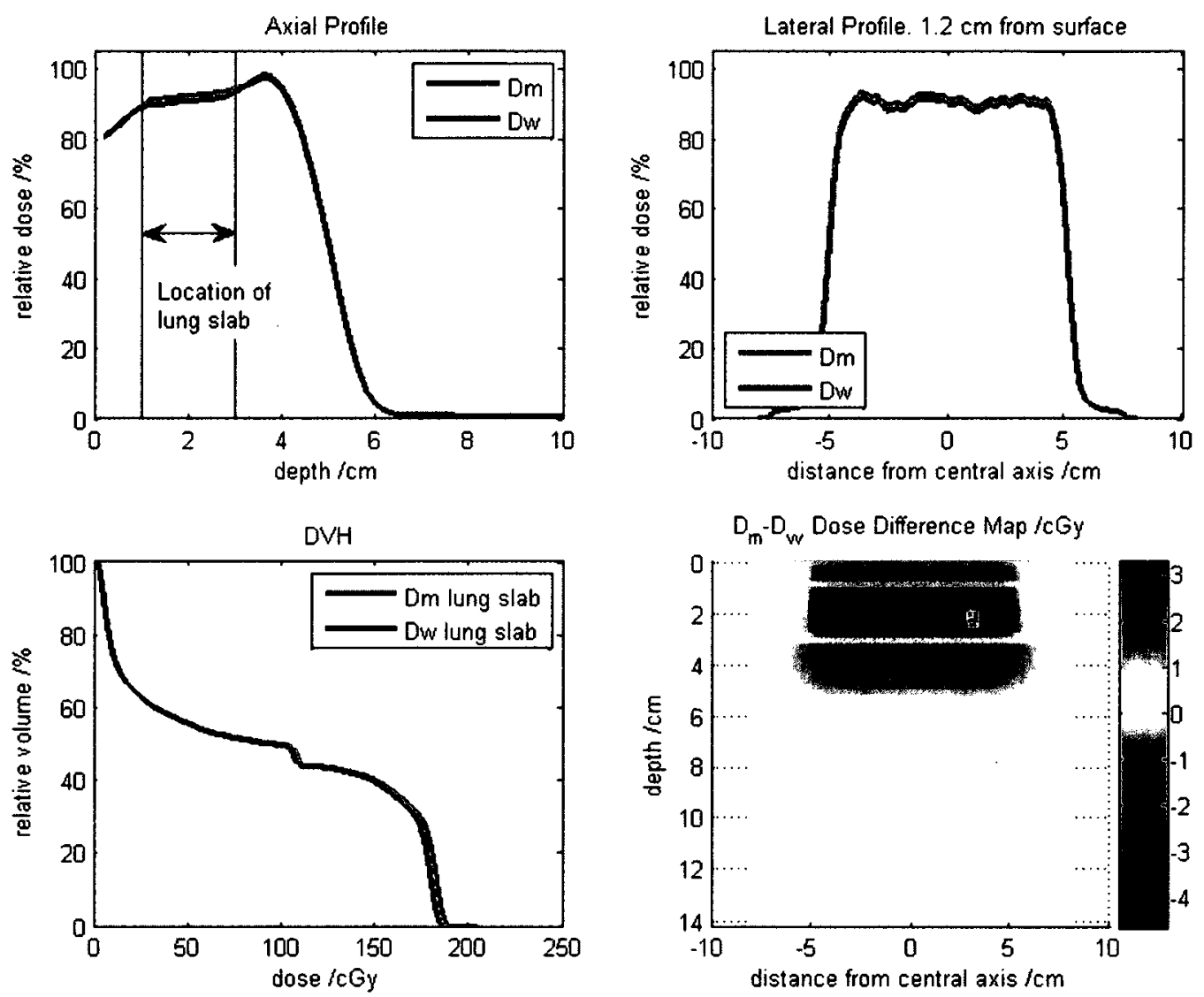

Figure 28. Axial and lateral dose profiles, DVH and dose difference map for the imagebased lung slab phantom, calculated to deliver $200 \mathrm{MU}$ with a $9 \mathrm{MeV}$ beam. Profiles were normalized to $D_{\max }$ in a homogenous water phantom.

The greatest difference between the $9 \mathrm{MeV}$ lung slab axial dose calculations is $1.6 \%$ (3.2 cGy). The DVH in Figure 28 shows that the overall dose calculated within the lung slab is very similar for both calculation approaches. However, within the lung slab, there is a section where the lung is denser as is evident on the CT image. This difference is particularly evident in the dose difference map for the $9 \mathrm{MeV}$ lung slab calculations. The dose calculated to water at this point is roughly $4 \mathrm{cGy}$ larger than dose-to-medium. 

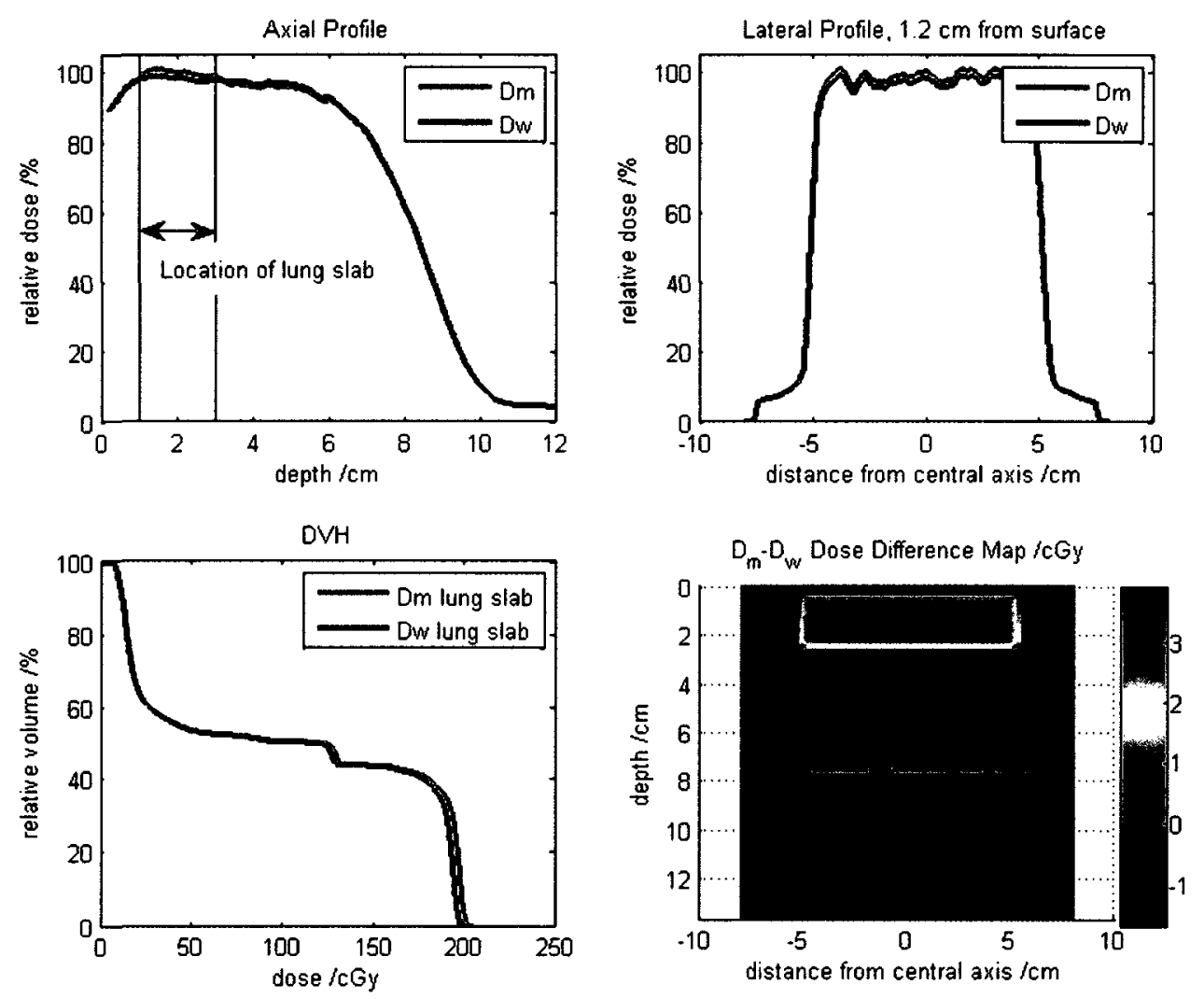

Figure 29. Axial and lateral dose profiles, DVH and dose difference map for the imagebased lung slab phantom, calculated to deliver $200 \mathrm{MU}$ with a $17 \mathrm{MeV}$ beam. Profiles were normalized to $D_{\max }$ in a homogenous water phantom.

The differences seen in the calculations for the $17 \mathrm{MeV}$ lung slab phantom are similar for those seen for the $9 \mathrm{MeV}$ phantoms. In the axial dose profile, the differences between the $\mathrm{D}_{\mathrm{m}}$ and $\mathrm{D}_{\mathrm{w}}$ calculations are consistently around $1.6 \%(3.2 \mathrm{cGy})$ within the lung slab. The lateral dose profiles, taken at a depth of $1.2 \mathrm{~cm}$, did not differ by more than $1.8 \%$ (3.6 cGy). The good agreement between the dose calculations translates to the DVH; the $\mathrm{D}_{\mathrm{m}}$ and $\mathrm{D}_{\mathrm{w}}$ DVHs for the lung slab phantom are very similar.

The differences between the $D_{m}$ and $D_{w}$ calculations are consistent with the water-to-lung unrestricted mass collisional stopping powers within the range of energies used in the 
clinic, $\sim 0.985$. This ratio suggests that the $D_{m}$ would be higher than $D_{w}$, which in this case it is.

The axial dose profiles of the image and vector-based lung slab geometries are similar, see Figure 30. The profiles differ most along the dose fall off. The image-based plans have somewhat larger $R_{p}$, which is especially apparent for the higher energy beam. The dose at the phantom surface is somewhat lower for the image-based anatomies, and the maximum dose behind the lung slab is always lower for the vector-based anatomies. Differences between the image and vector-based profiles are due to several reasons. The most important is the difference in electron densities of these anatomies. For the vectorbased anatomy we use electron density 0.3 , based on the specifications provided by the manufacturer of the phantom (RMI) and White et $a l^{30}$. The electron density for the image-based anatomy is given by the CT calibration curve and ranges between 0.28 and 0.31. These somewhat different electron density values are mostly responsible for the differences observed in Figure 30. Other reasons could be non-uniform density of the physical phantoms, and tiny air gaps between the slabs. 


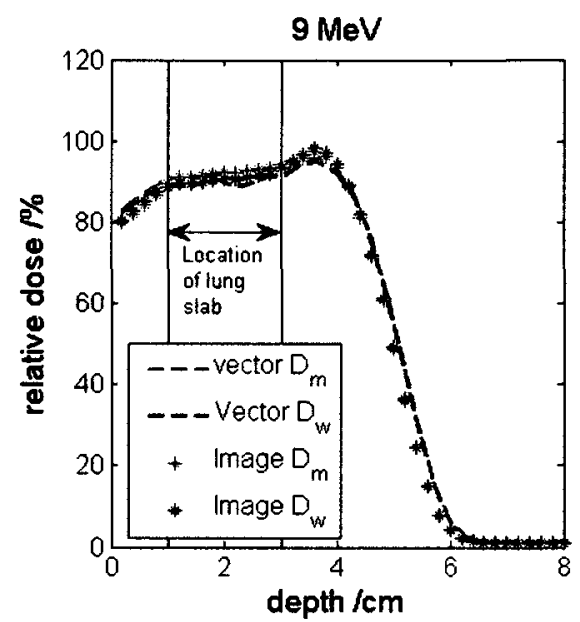

(a)

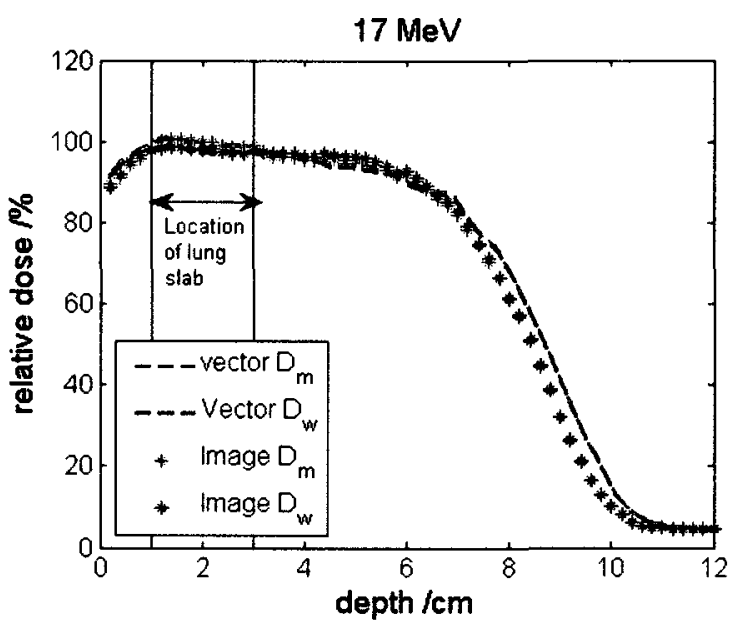

(b)

Figure 30. Axial dose profiles of the vector-based and image-based lung slab phantom for (a) $9 \mathrm{MeV}$ and (b) $17 \mathrm{MeV}$. Profiles were normalized to $D_{\max }$ in a homogenous water phantom.

\subsubsection{Results: Hard bone cylinder epoxy resin image-based phantom}

The 9 and $17 \mathrm{MeV}$ hard bone cylinder phantom calculations show similar differences between doses calculated using the $D_{m}$ and $D_{w}$ approaches. Profiles for these phantoms are presented in Figure 31 and Figure 32. 

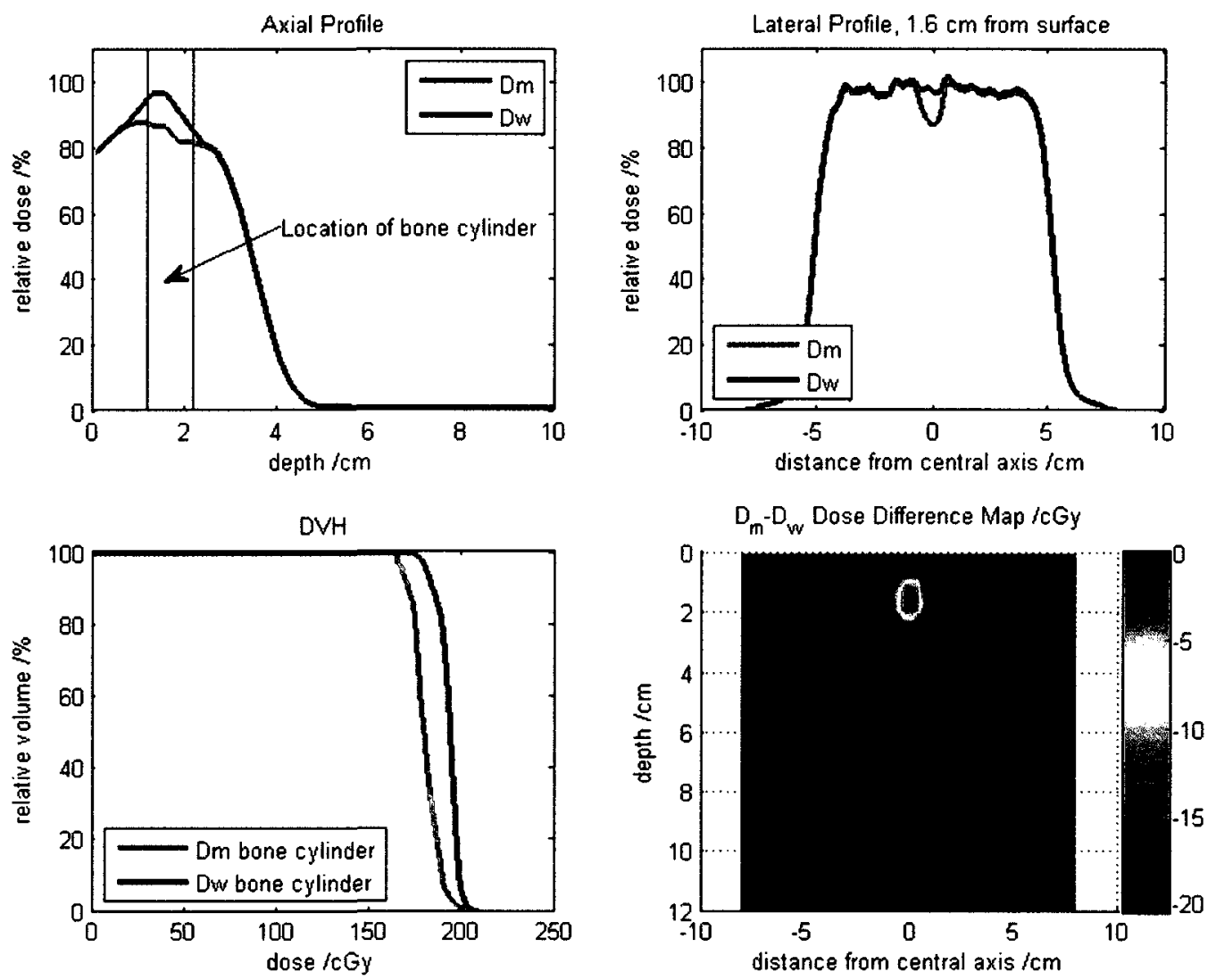

Figure 31. Axial and lateral dose profiles, DVH and dose difference map for the imagebased hard bone cylinder phantom, calculated to deliver $200 \mathrm{MU}$ with a $9 \mathrm{MeV}$ beam. Profiles were normalized to Dmax in a homogenous water phantom.

Along the axial dose profile, the largest difference between the $17 \mathrm{MeV} \mathrm{D}_{\mathrm{m}}$ and $\mathrm{D}_{\mathrm{w}}$ calculations was $10.2 \%$ (20.4 cGy) of the maximum dose. This maximum difference occurred at a depth of $1.6 \mathrm{~cm}$ from the surface, near the center of the bone cylinder. Within and around the bone cylinder, the $D_{w}$ was larger than $D_{m}$. The difference between $D_{w}$ and $D_{m}$ along the axial dose profile within the hard bone cylinder ranged between $6.9 \%$ and $10.2 \%$. The smaller differences exist near the boundaries of the hard bone cylinder. The discrepancies could be due to the fact that the edges of the bone cylinder are within voxels that encompass both hard bone and water, as shown in Figure 33. The 
black grid represents the calculation matrix, and the green grid is the display matrix. The blue box is representative of the bone heterogeneity. This could also contribute to the differences between the $D_{m}$ and $D_{w}$ axial dose profiles on the outside boundaries of the bone cylinder.
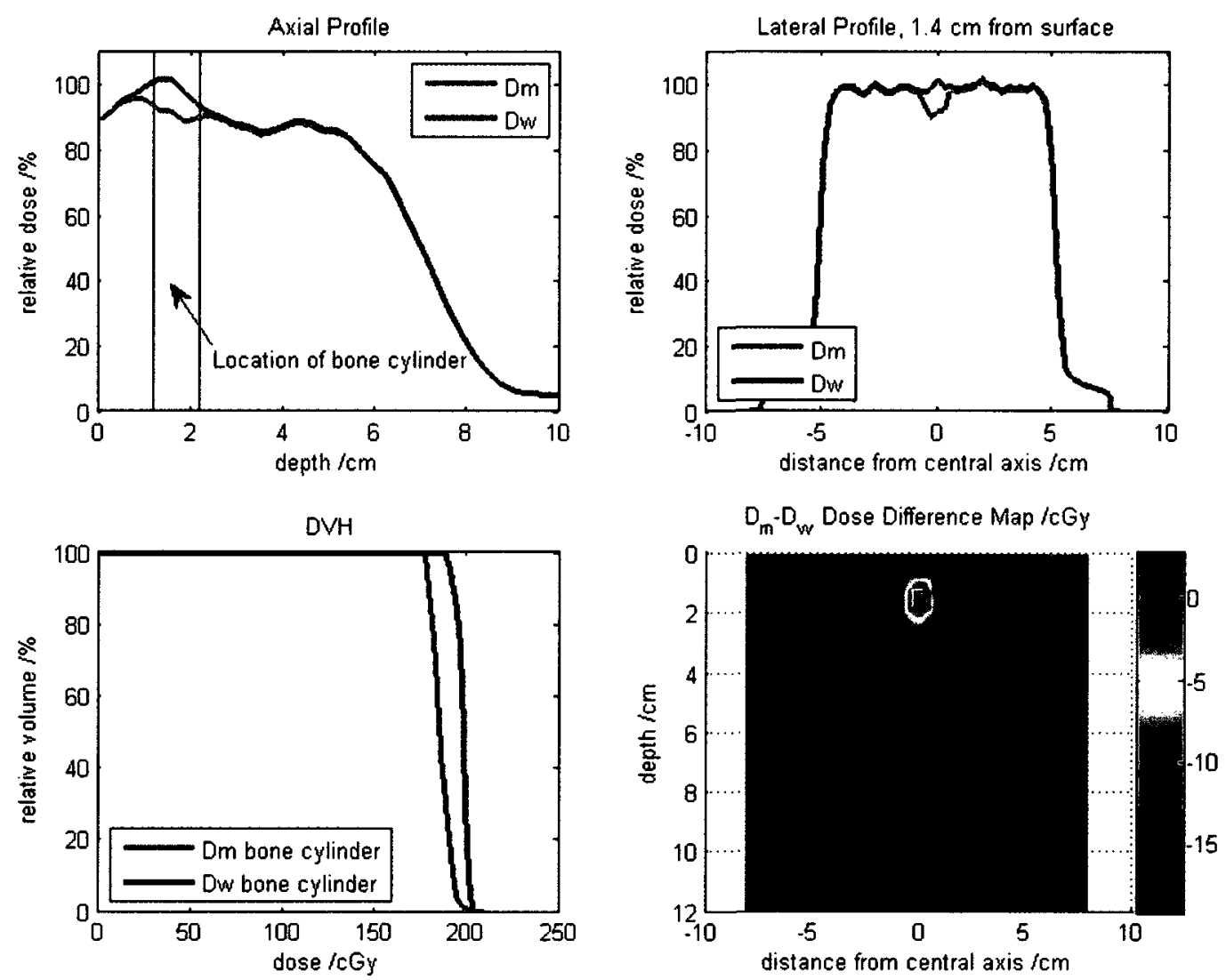

Figure 32. Axial and lateral dose profiles, DVH and dose difference map for the imagebased hard bone cylinder phantom, calculated to deliver $200 \mathrm{MU}$ with a $17 \mathrm{MeV}$ beam. Profiles were normalized to Dmax in a homogenous water phantom.

The lateral dose profiles were taken through the bone, at a depth of $1.6 \mathrm{~cm}$ from the surface. Unlike the $D_{m}$ calculations, the $D_{w}$ lateral dose profile does not show the existence of the bone heterogeneity within the field. However, for the $D_{m}$ calculations, 
there is a clear distinction of where the bone cylinder is along the $D_{m}$ lateral dose profile; the $D_{m}$ calculations at the point of the heterogeneity was approximately $10.3 \%$ lower than the $D_{w}$ calculated values. The DVH for the hard bone cylinder shows constant differences for $D_{m}$ and $D_{w}$ calculations over the entire volume.

For the $17 \mathrm{MeV}$ bone cylinder phantoms, the difference between doses calculated within the bone cylinder phantom are only marginally smaller than for the $9 \mathrm{MeV}$ phantoms. For the $17 \mathrm{MeV}$ bone cylinder phantom, the largest difference between the two calculations along the axial dose profile was $9.5 \%(19.3 \mathrm{cGy})$, and it occurred $1.4 \mathrm{~cm}$ from the phantom's surface. As is the case of all the bone cylinder phantom calculations, the presence of bone along the lateral dose profile is only seen in the $D_{m}$ calculations. The $17 \mathrm{MeV} \mathrm{D}_{\mathrm{m}}$ lateral dose profiles taken $1.4 \mathrm{~cm}$ from the phantom surface is lower than the $D_{w}$ lateral dose profile by $9.6 \%(19.3 \mathrm{cGy})$ in the area where bone is present.

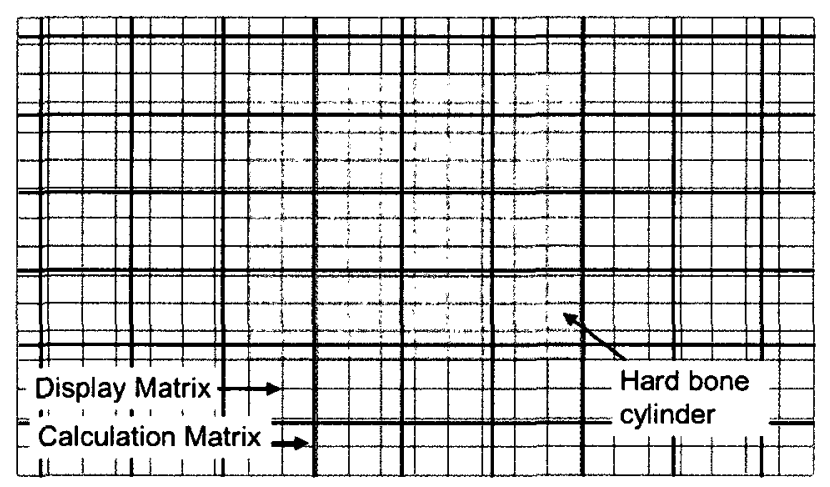

Figure 33. Display matrix superimposed on the calculation matrix and the image-based bone cylinder phantom.

When the axial dose profiles of the image and vector-based plans were compared, there was an obvious difference between profiles, see Figure 34 . The largest differences 
between the vector and image based phantoms occur within the inhomogeneity and just after it. Differences like these are not seen in any of the other anatomies. This clearly indicates that there are limitations to using a vector-based anatomy to represent a physical phantom. Differences in densities, scattering powers, the effects of having tiny air gaps between the slabs, the resolution of the phantom are all limiting factors that could be causing the discrepancies between the vector and image-based axial dose profiles.

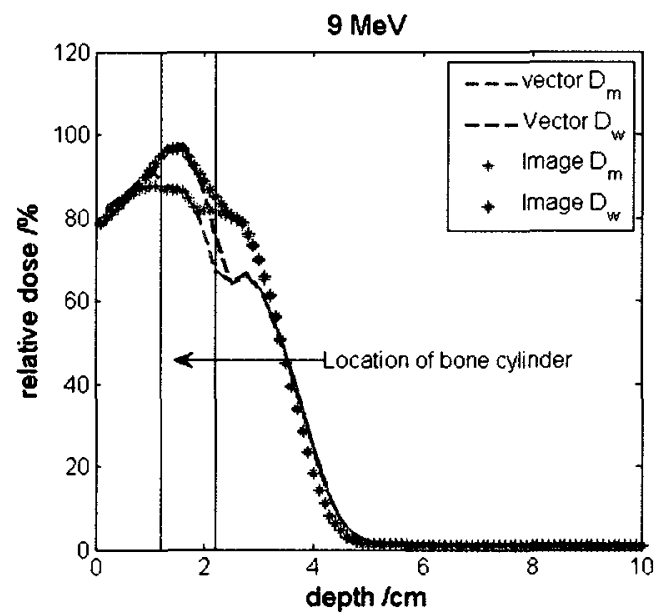

(a)

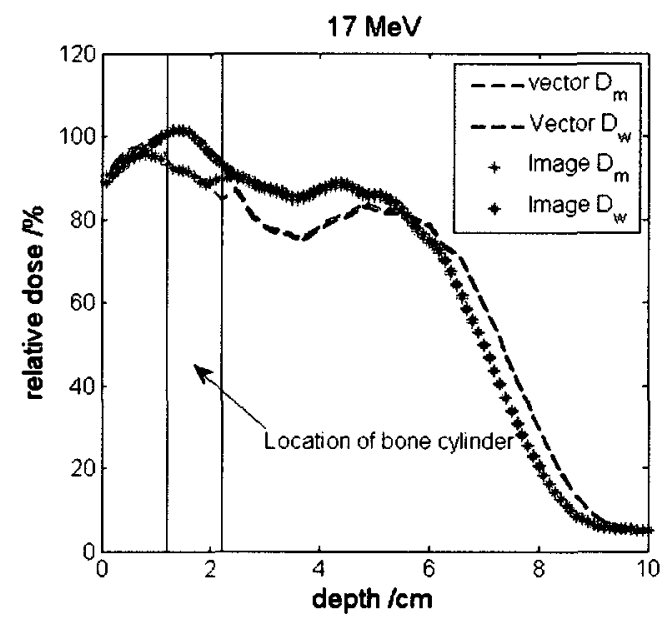

(b)

Figure 34. Axial dose profiles of the vector-based and image-based hard bone cylinder phantom for (a) $9 \mathrm{MeV}$ and (b) $17 \mathrm{MeV}$. Profiles were normalized to $D_{\max }$ in a homogenous water phantom.

\subsubsection{Results: Air cylinder epoxy resin image-based phantom}

The $9 \mathrm{MeV}$ air cylinder image-based phantom calculations are shown in Figure 35. The $D_{m}$ and $D_{w}$ calculations are in very good agreement, especially in the water surrounding the air cavity. Along the axial dose profile where the air cylinder is present, the differences between the calculation methods gradually become larger with increasing 
depth. This trend was also visible in the $9 \mathrm{MeV}$ vector-based air cylinder phantom. For the $9 \mathrm{MeV}$ image-based phantom axial dose calculations, the largest difference between $D_{m}$ and $D_{w}$ is $5.3 \%(10.6 \mathrm{cGy})$ at $2.7 \mathrm{~cm}$ from the surface. This is the same result as for the analogous vector based phantom.
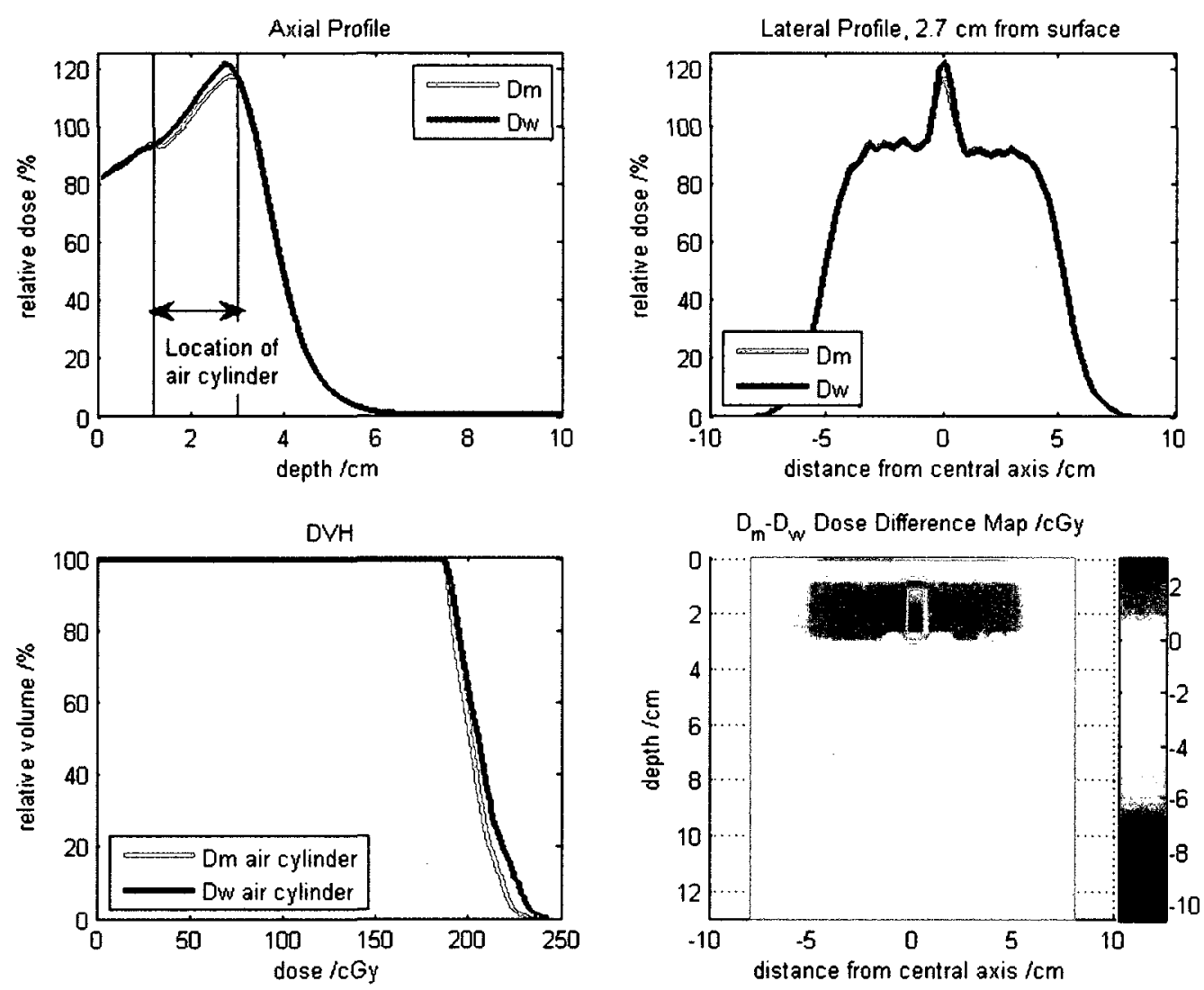

Figure 35. Axial and lateral dose profiles, DVH and dose difference map for the imagebased air cylinder phantom, calculated to deliver $200 \mathrm{MU}$ with a $9 \mathrm{MeV}$ beam. Profiles were normalized to $D_{\max }$ in a homogenous water phantom.

At depths 1 and $2 \mathrm{~cm}$ in the dose difference map there is a visible step in dose. The dose distribution is not as gradual as seen in the vector-based phantom. These dose steps are located at the boundaries of the MS11 slab containing the air cavity. When further examined, it was found this particular slab had a slightly lower electron density than all 
the other slabs used in this study. As seen in the phantoms discussed thus far, when the electron density of a material is lower than water (like in lung), the $D_{m}$ is higher than $D_{w}$. This can be seen in the dose difference map.

Unlike the $9 \mathrm{MeV}$ calculations, the difference between the $D_{m}$ and $D_{w}$ axial dose calculations for the $17 \mathrm{MeV}$ plan do not increase with depth. The largest difference along this profile was $1.9 \%$ at $1.4 \mathrm{~cm}$ from the surface $(0.2 \mathrm{~cm}$ within the air cylinder).

For the lateral dose profile taken at $1.4 \mathrm{~cm}$ from the surface, the $\mathrm{D}_{\mathrm{m}}$ is larger for the points of the profile that lie around the location of the air cylinder but smaller within the air cavity. The greatest difference between the lateral dose profiles is $1.9 \%$ at the central axis. The $17 \mathrm{MeV} \mathrm{D}_{\mathrm{m}}$ and $\mathrm{D}_{\mathrm{w}}$ calculations were in better agreement with each other than those for the $9 \mathrm{MeV}$ plans. 

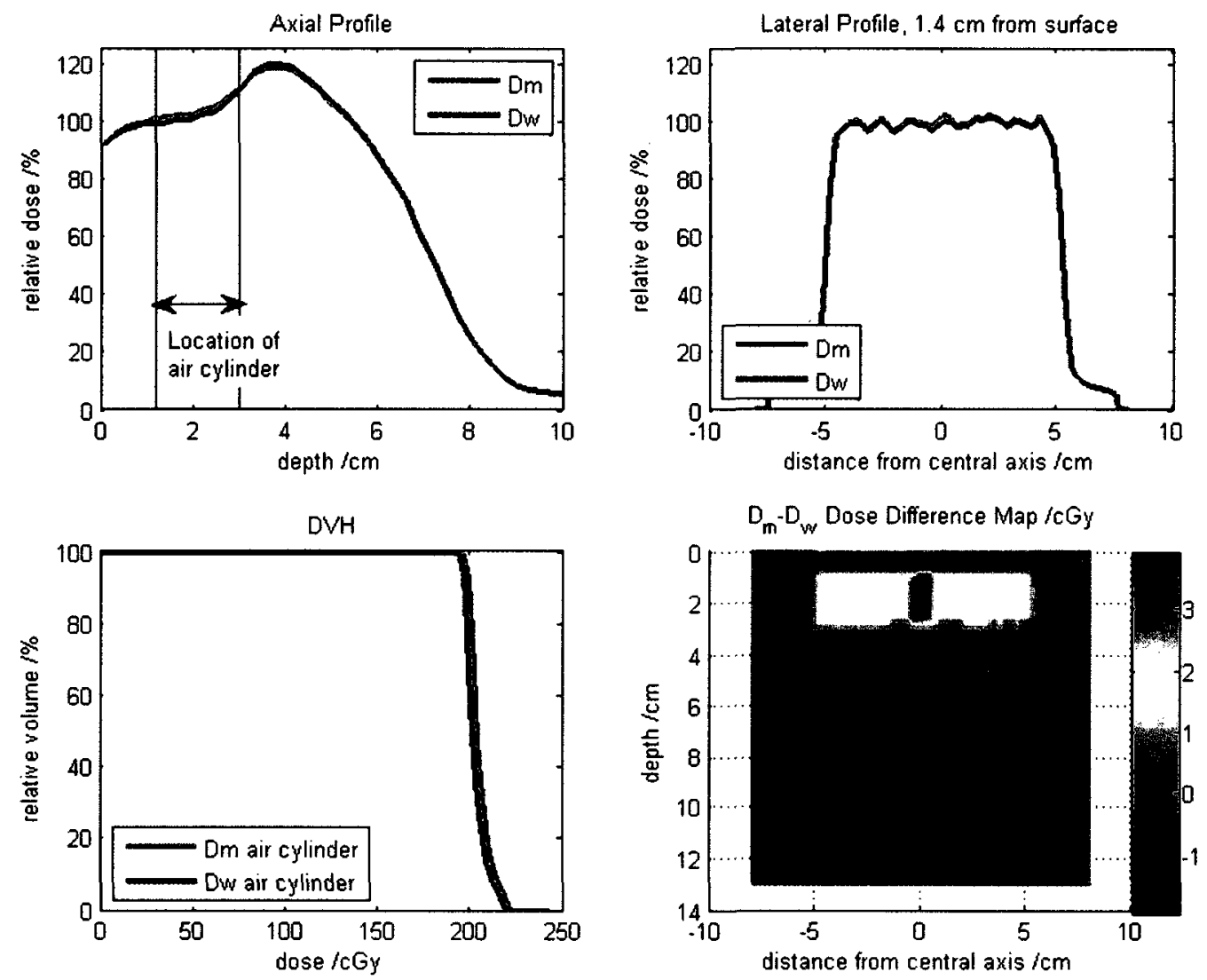

Figure 36. Axial and lateral dose profiles, DVH and dose difference map for the imagebased air cylinder phantom, calculated to deliver $200 \mathrm{MU}$ with a $17 \mathrm{MeV}$ beam. Profiles were normalized to $D_{\max }$ in a homogenous water phantom.

\subsubsection{Results: Trachea and spine epoxy resin image-based phantom}

The dose profiles, DVH and the dose difference maps are presented in Figure 37 and Figure 38 for the 9 and $17 \mathrm{MeV}$ calculations, respectively. For clarity, the DVHs for only two of the five inhomogeneities, the air cylinder and the bone cylinder centered at $-2.75 \mathrm{~cm}$ from the central axis are displayed.

The $D_{m}$ and $D_{w} 9 \mathrm{MeV}$ axial dose profile through trachea and spine phantoms have a maximum difference of $3.9 \%(7.8 \mathrm{cGy})$ at a depth of $3.6 \mathrm{~cm}$ from the surface, within the 
air cylinder. The $D_{w}$ is higher than the $D_{m}$. For the lateral dose profile taken at $3.6 \mathrm{~cm}$ from the surface, the $D_{w}$ is larger. The greatest difference between the lateral dose profiles is $3.9 \%(7.8 \mathrm{cGy})$.
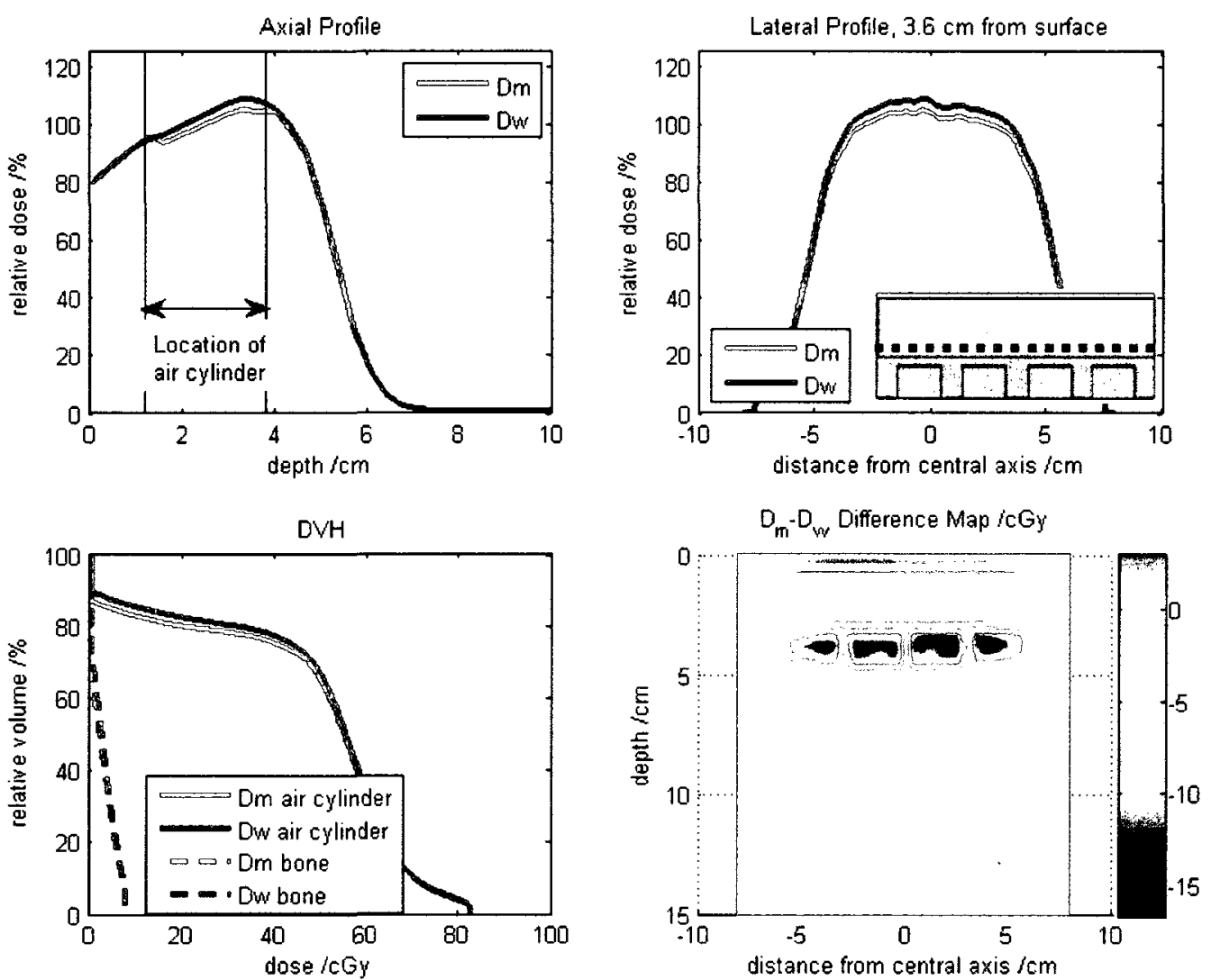

Figure 37. Axial and lateral dose profiles, DVH and dose difference map for the imagebased trachea \& spine phantom, calculated to deliver $200 \mathrm{MU}$ with a $9 \mathrm{MeV}$ beam. The lateral dose profile contains a pictorial description of where the profile was taken within the phantom. Profiles were normalized to $D_{\max }$ in a homogenous water phantom.

The calculations for the $17 \mathrm{MeV}$ trachea and spine phantom calculations were in better agreement than those for the $9 \mathrm{MeV}$ plans. The greatest difference between the calculations along the axial dose profile was $1.9 \%(3.6 \mathrm{cGy})$ at $1.6 \mathrm{~cm}$ from the surface. This difference between $D_{m}$ and $D_{w}$ was consistent along the lateral dose profile taken at 
$1.2 \mathrm{~cm}$ from the surface; the maximum difference between the $D_{m}$ and $D_{w}$ calculations was $1.9 \%(3.6 \mathrm{cGy})$. The difference of dose at a dept of $5.7 \mathrm{~cm}$ can be attributed to the air gap between the spine and trachea slab and the back scatter assembly.
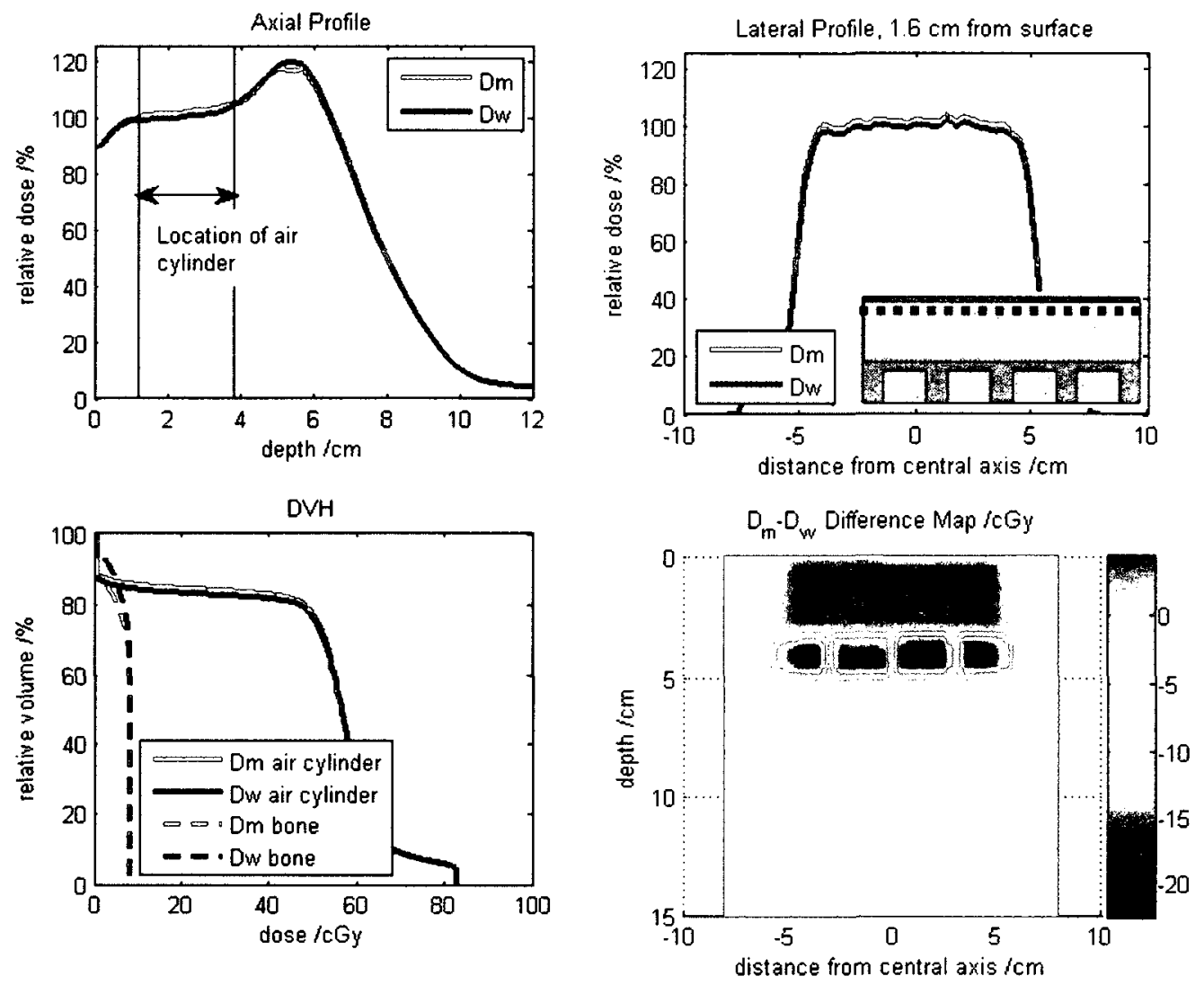

Figure 38. Axial and lateral dose profiles, DVH and dose difference map for the imagebased trachea \& spine phantom, calculated to deliver $200 \mathrm{MU}$ with a $17 \mathrm{MeV}$ beam. The lateral dose profile contains a pictorial description of where the profile was taken within the phantom. Profiles were normalized to $D_{\max }$ in a homogenous water phantom. 


\subsection{Measurements of dose in epoxy resin based phantoms using film}

The treatment plans were delivered to the ERB phantoms using a Siemens Primus linear accelerator, Serial \# 4173. The delivered dose was measured with EBT1 Gafchromic film (International Specialty Products, Wayne, New Jersey). Prior to using the film, each film batch used was calibrated. A single batch was used for all except for the trachea phantom. The lot \# of the batch used for the slab and cylindrical geometries was Lot \# 48022-02BI. The measurements in the trachea phantoms were done using EBT1 Gafchromic films Lot \# 48022-4BI.

\subsubsection{Calibration of the GafChromic Film}

Although some studies state that GafChromic film is independent of energy ${ }^{35}$, calibration curves were made for each batch and energy ( 9 and $17 \mathrm{MeV})$.

Each sheet of film was scanned prior to irradiation using an Epson Perfection V700 photo scanner. The response of the film is affected by the orientation with which the film is scanned ${ }^{36}$ and irradiated. Therefore, care was taken to scan each sheet of film in the same orientation by marking the top left corner with a dot using a fine-tipped permanent marker. Once the sheet had been scanned, it was cut into $2 \times 3 \mathrm{~cm}^{2}$ pieces, each piece was marked with a dot so that it was possible to preserve the original film orientation.

Each piece of film was placed at $d_{\max }$ in homogenous phantom and exposed to a $10 \times 10$ $\mathrm{cm}^{2}$ beam at an SSD of $100 \mathrm{~cm}$. Using the dot on the film, care was taken to keep each piece of film in the same orientation with respect to the linac. Each film measurement was made twice. Two pieces of film received the following monitor units for each energy: $10,20,30,50,80,100,200,300,400$, and 500 MU. Reference doses were 
determined using an IBA-Scanditronix NACP-02 plane parallel chamber (Louvain-laNeuve, Belgium), and the TG-51 protocol ${ }^{4}$.

The homogeneous phantom is composed of several $30 \times 30 \mathrm{~cm}^{2}$ slabs of of MS11. The depth of maximum dose was determined using the PDDs of the 9 and $17 \mathrm{MeV}$ beam calibration data. The set up for each piece of film was the same. The film was placed along the isocenter at $d_{\max }$ within the phantom. The Siemens Linac used to irradiate the phantoms is calibrated such that $100 \mathrm{MU}$ corresponds to $100 \mathrm{cGy}$ at $\mathrm{d}_{\max }$ in a homogeneous water phantom.

Due to the increase of optical density with time post irradiation ${ }^{37}$, the film was allowed to stabilize for twenty-four hours. The irradiated pieces of film were scanned with the same Epson scanner in the same orientation as the unirradiated film. The scanned images of the unirradiated and irradiated film were imported into Film QA (3COGNITION LLC), a software program used for quantitative film analysis. This program was used to obtain the average pixel values of each film piece image. The pixel values were converted to a net optical density using the following equation ${ }^{38}$ :

$$
n e t O D=\log _{10}\left(I_{\text {unirad }} / I_{\text {irad }}\right)
$$

where $I_{\text {unirad }}$ is the pixel value of the film prior to irradiation and $I_{\text {irad }}$ is the pixel value of the film after it has been irradiated. The data of the net optical densities and their corresponding reference doses were fitted, using least-square methods, to the following function by Devic et $a l^{38}$ : 


$$
D_{f i t}=a+b \cdot n e t O D+c \cdot n e t O D^{n}
$$

Parameters $a, b, c$, and $n$ were obtained for each batch and energy. The 9 and $17 \mathrm{MeV}$ calibration curves are presented in Figure 39. The calibration curves are in close agreement with each other. Our film data confirm the EBT1 film response is almost independent of beam energy. However, we use energy specific calibration curves to increase the accuracy of our measurements.

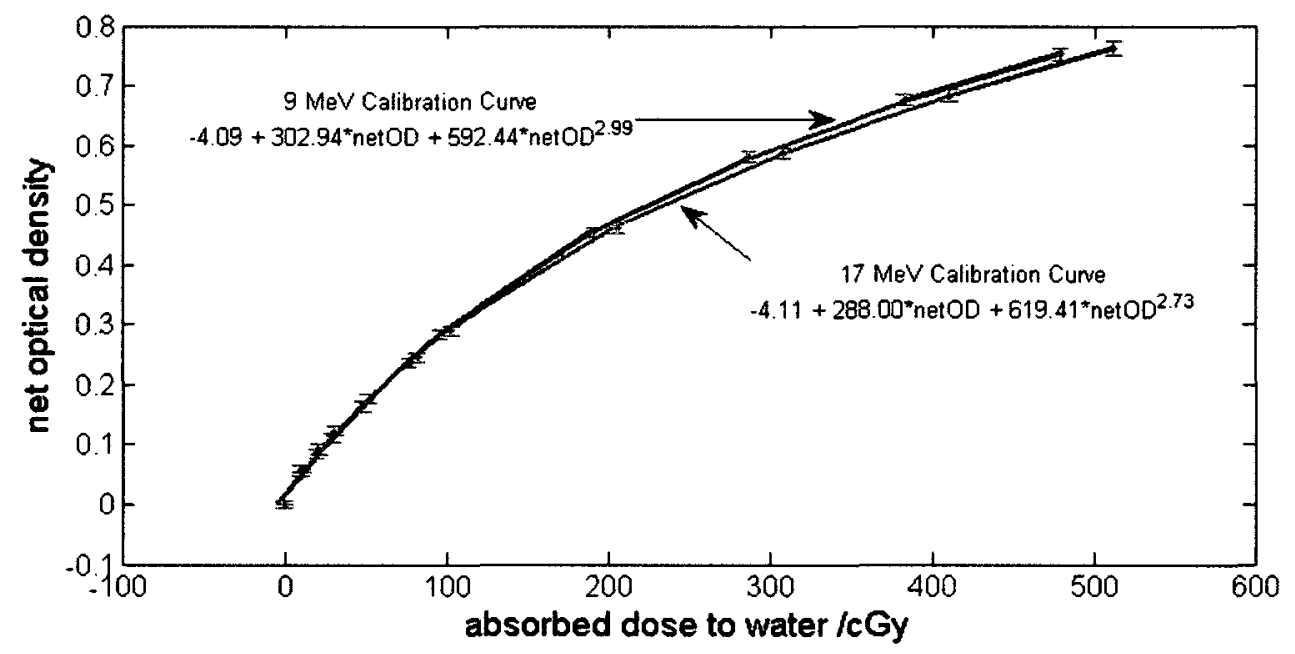

Figure 39. $9 \mathrm{MeV}$ and $17 \mathrm{MeV}$ GafChromic EBTl film calibration curve.

\subsubsection{Film setup within the epoxy resin based phantoms}

For measurements within the slab and small cylindrical inhomogeneity phantoms, slices of $15 \times 6 \mathrm{~cm}^{2}$ pieces of film were prepared. For the trachea and spine phantom the film was cut into $15 \times 15 \mathrm{~cm}^{2}$ pieces. As per the procedure outlined in section 6.4 .1 , the films were scanned prior to being cut and irradiated. For each measurement, the pieces of film were centered in the phantom and using one piece of film at a time, the phantom was 
irradiated with the prescribed number of MUs. The phantoms used for film measurements were the same as those used in the imaged-based anatomy calculations, with the exception that two $1 \mathrm{~cm}$ MS11 slabs were added to the top of the backscatter assembly. This allowed for the film to be placed at different depths within the phantom. The depths the pieces of the film were placed at are outlined as follows:

\section{Slab Geometry of Bone and Lung}

For the two phantoms containing the lung and bone slab inhomogeneities, eight measurements were taken for each phantom. Two measurements were made at each of the four locations within the phantom. These locations are indicated in Figure 40: (1) above the slab inhomogeneity, (2) at the center of the slab inhomogeneity, (3) directly below the slab inhomogeneity and (4) $1 \mathrm{~cm}$ below the slab inhomogeneity.

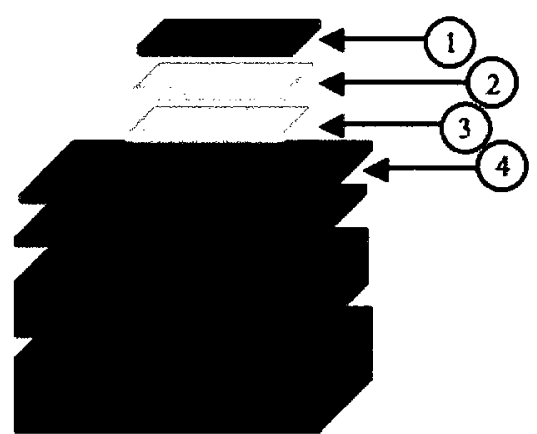

Figure 40. Location of GafChromic EBT1 film within slab geometry phantoms.

\section{Small Cylindrical Geometries}

For the phantom containing the small cylindrical inhomogeneities four measurements were taken: two measurements at each of two locations indicated in Figure 41. During measurements, the exposed side of the cylindrical 
inhomogeneity was face down and the film was placed (1) directly below the slab containing the cylindrical inhomogeneity, in contact with the inhomogeneity, and (2) $1 \mathrm{~cm}$ below the inhomogeneity.

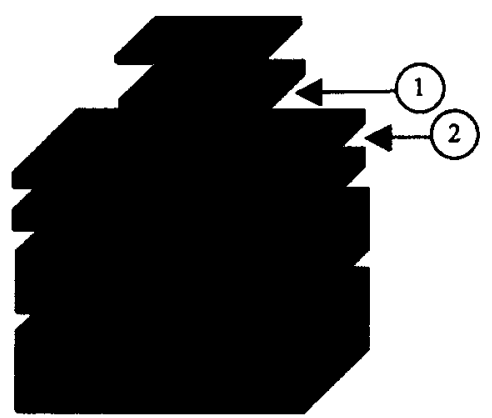

Figure 41. Location of the GafChromic EBT1 film within the hard bone and air cylinder phantoms.

\section{Trachea and Spine}

Four measurements were taken in the trachea and spine phantom. Two measurements were taken at the depths specified in Figure 42: (1) directly below the trachea and spine slab, where the film was in contact with the inhomogeneity, (2) $1 \mathrm{~cm}$ below trachea and spine slab.

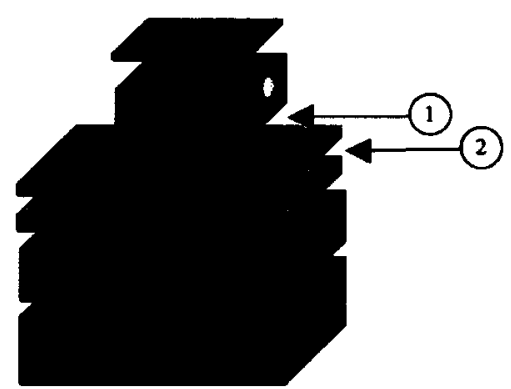

Figure 42. Location of the GafChromic EBT1 film within the trachea and spine phantom. 
Immediately after irradiation, the film was stored in a cool, dark location. After allowing the film to stabilize for twenty-four hours, the films were rescanned on the same Epson scanner, and analyzed with the Film QA software. The film dose profiles were evaluated by obtaining lateral pixel value profiles across the center of the film and converting them to dose using the calibration curves ${ }^{38}$ discussed in the previous section.

\subsubsection{Results: Film measurements}

With the exception of the slab geometries, the film was always in MS11. It was expected that the film would agree best with the $D_{w}$ image-based calculations, since the film was calibrated to dose-to-water and image-based anatomy is a more accurate representation of the real phantom than the vector-based anatomies. The film measurements, when compared with the $D_{m}$ and $D_{w}$ calculations of the image and vector-based anatomies, did not show any trend to agree with either the $D_{m}$ or $D_{w}$ calculations for either type of anatomy. Shown here are only the results for the 9 and $17 \mathrm{MeV}$ bone and lung slab phantoms. The relative uncertainties of the doses are larger for lower doses. The film dose measurements had uncertainties of as high as $11 \%$ for doses measured at points of interest.

Since the difference between $D_{m}$ and $D_{w}$ is roughly $10 \%$ in areas of hard bone, we would expect to be better able to see if the film measurements agree better with one calculation approach over another. Figure 43 presents the $9 \mathrm{MeV}$ lateral dose profiles of the film measurements, and TPS calculations at different depths of the vector and image based epoxy resin phantom. For the most part, the positions of the vector and image-based calculations with respect to the film measurements were the same for each depth. 
$9 \mathrm{MeV}$ Bone Slab Phantom Film Measurements and

Vector based Calculations

\begin{tabular}{|l|}
\hline Directly above \\
bone slab, 1.0 \\
cm from \\
phantom \\
surface \\
\hline
\end{tabular}

In the middle of bone slab $1.5 \mathrm{~cm}$ from phantom surface

Directly below bone slab. $2.0 \mathrm{~cm}$ from phantom surface

$1 \mathrm{~cm}$ below bone slab. $3.0 \mathrm{~cm}$ from phantom surface
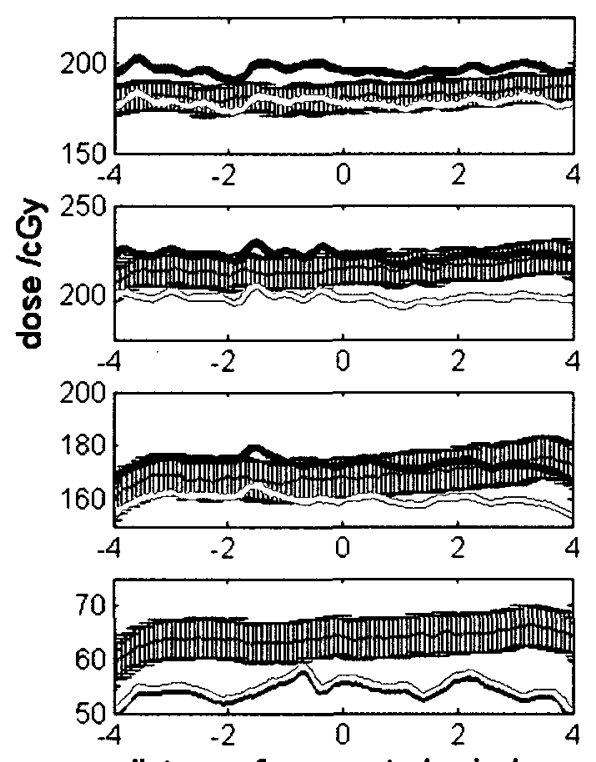

distance from central axis $/ \mathrm{cm}$
Image Based Calculations
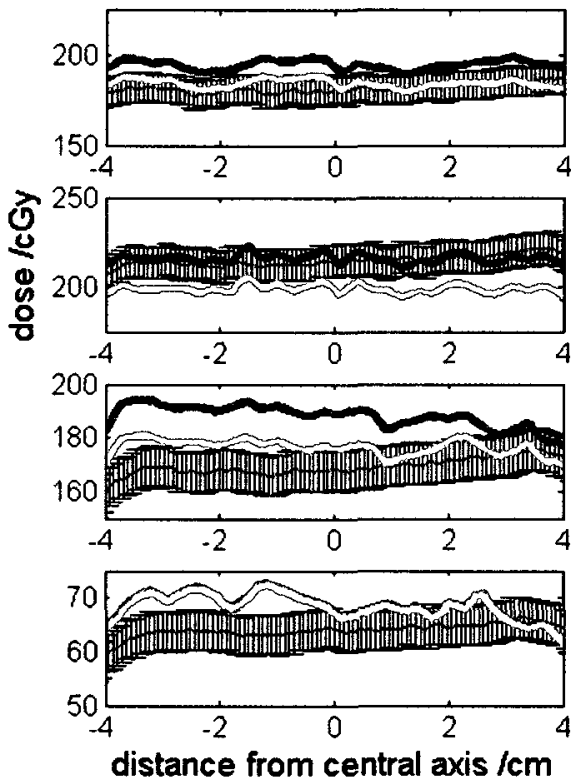

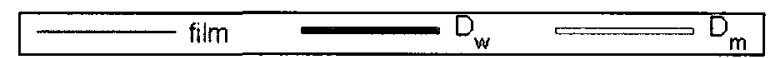

Figure 43. $9 \mathrm{MeV}$ film measurements and calculations at various depths within the hard bone slab phantom. To the left are the film measurements and vector based calculations, to the right are the film measurements and the image based calculations.

For the $9 \mathrm{MeV}$ profiles taken 1.0 and $1.5 \mathrm{~cm}$ from the surface, there seems to be a fair agreement with the calculated and measured values. However, the measurements do not tend to agree more with one calculation method or another. Taking into consideration all the profiles, the $17 \mathrm{MeV}$ calculated profiles shown in Figure 44, have better agreement with the measured doses. 
$17 \mathrm{MeV}$ Bone Slab Phantom Film Measurements and

Vector based Calculations

\begin{tabular}{|l|}
\hline Directly above \\
bone slab. 1.0 \\
cm from \\
phantom \\
surface \\
\hline
\end{tabular}

In the middle of bone slab $1.5 \mathrm{~cm}$ from phantom surface

Directly below bone slab.

$2.0 \mathrm{~cm}$ from

phantom

surface

$1 \mathrm{~cm}$ below

bone slab.

$3.0 \mathrm{~cm}$ from

phantom

surface
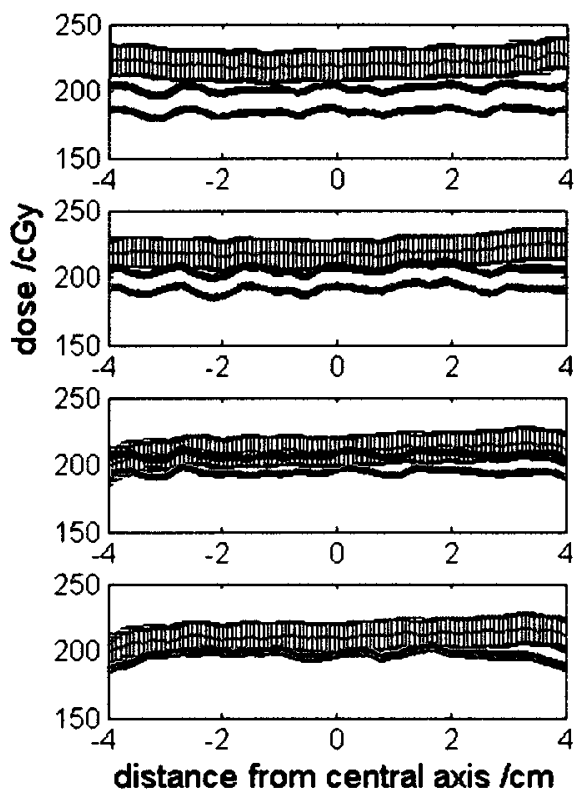

Image Based Calculations
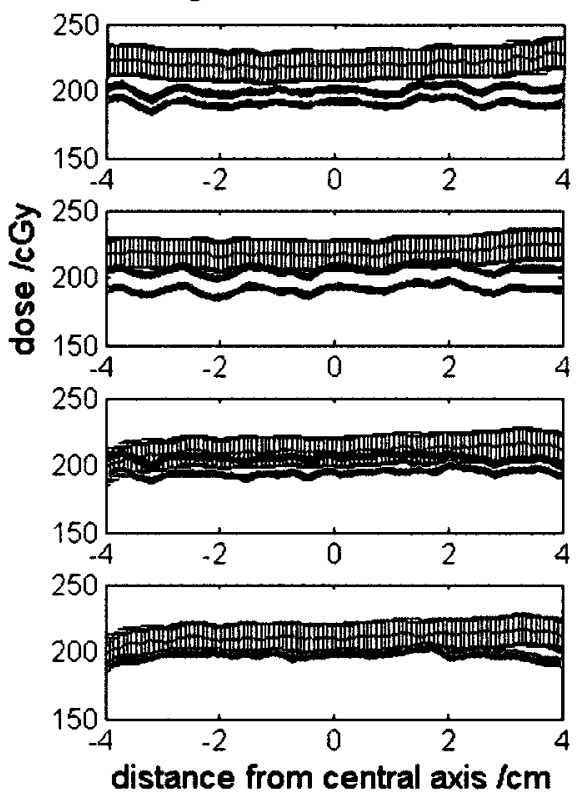

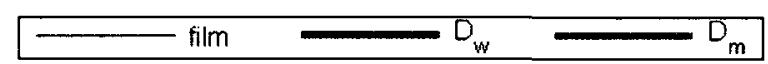

Figure 44. $17 \mathrm{MeV}$ film measurements and calculations at various depths within the hard bone slab phantom. To the left are the film measurements and vector based calculations, to the right are the film measurements and the image based calculations.

The agreement between the $17 \mathrm{MeV} \mathrm{D}_{\mathrm{m}}$ and $\mathrm{D}_{\mathrm{w}}$ calculations and measured profiles improves with depth. However, the film measurements tend to be higher than the calculated values.

The differences between the $D_{m}$ and $D_{w}$ seen in the phantoms containing low-density inhomogeneities, such as lung and air, do not show differences in excess of $6 \%$ to one another relative to $D_{\max }$ in a homogeneous water phantom. This makes it particularly difficult to determine if the film measurements agree better with the $D_{w}$ calculation approach, since the uncertainties of the film are larger than the differences seen between the $D_{m}$ and $D_{w}$ calculations. Figure 45 and Figure 46 are the film measurements and 
calculations at various depths within the lung slab phantom for the 9 and $17 \mathrm{MeV}$ plans, respectively.

$9 \mathrm{MeV}$ Lung Slab Phantom Film Measurements and

Directly above lung slab, 1.0 $\mathrm{cm}$ from phantom surface

In the middie of lung slab. $2.0 \mathrm{~cm}$ from phantom surface

\begin{tabular}{|l|}
\hline Directly below \\
lung slab. \\
$3.0 \mathrm{~cm}$ from \\
phantom \\
surface \\
\hline
\end{tabular}

\begin{tabular}{l}
$1 \mathrm{~cm}$ below \\
lung slab. \\
$4.0 \mathrm{~cm}$ from \\
phantom \\
surface \\
\hline
\end{tabular}

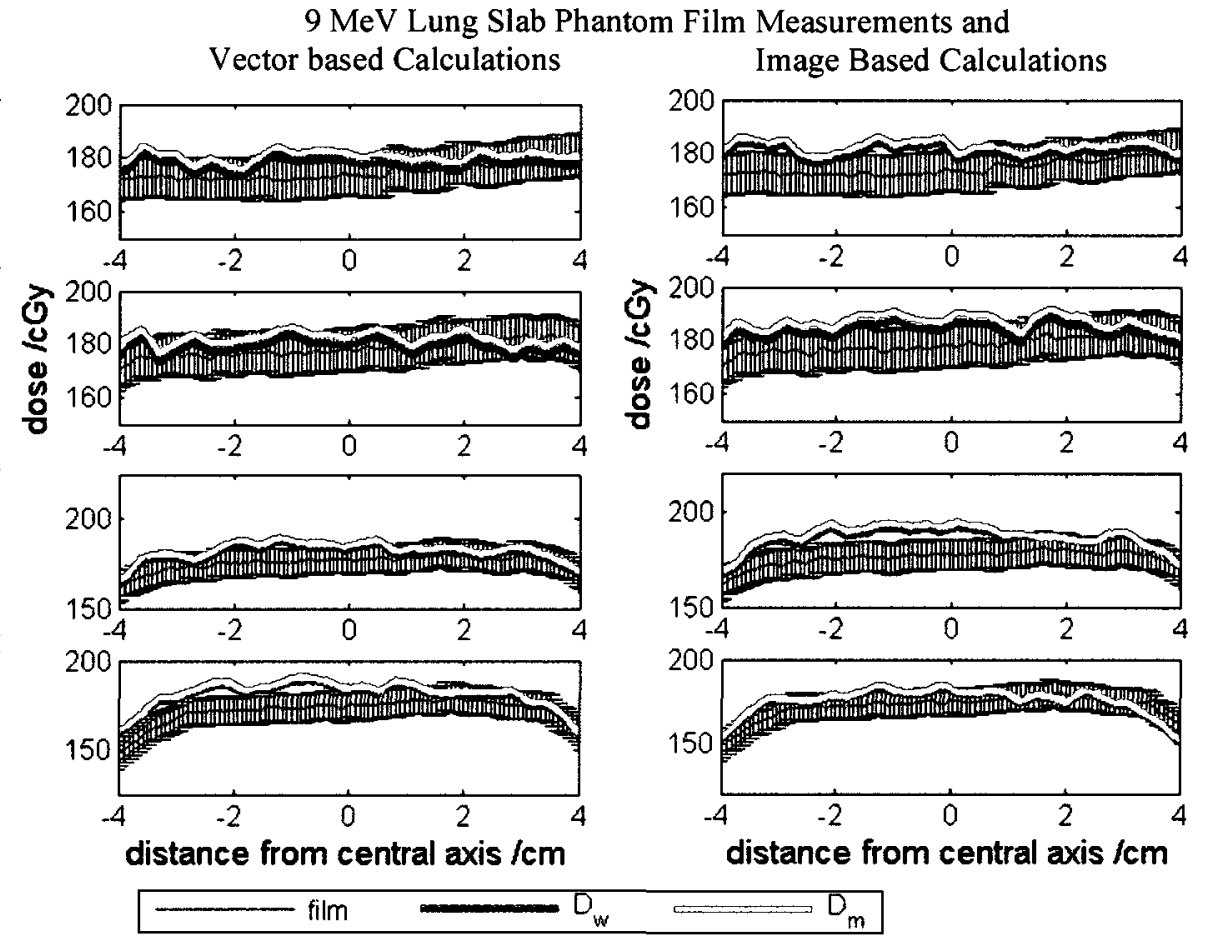

Figure 45. 9 MeV film measurements and calculations at various depths within the lung slab phantom. To the left are the film measurements and vector based calculations, to the right are the film measurements and the image based calculations.

For the $9 \mathrm{MeV}$ lung slab, there is a better agreement between the vector-based calculations and the measured values, than there is for the image-based dose profiles. By the most part, both the vector and image based calculations lie within the uncertainties of the film measurements. However, since both $D_{m}$ and $D_{w}$ lie within the uncertainties, it cannot be concluded that the dose-to-water calculations are in better agreement with the measurements inside the lung inhomogeneity. 
17 MeV Lung Slab Phantom Film Measurements and

Vector based Calculations

Image Based Calculations

Directly above
lung slab. 1.0
cm from
phantom
surface
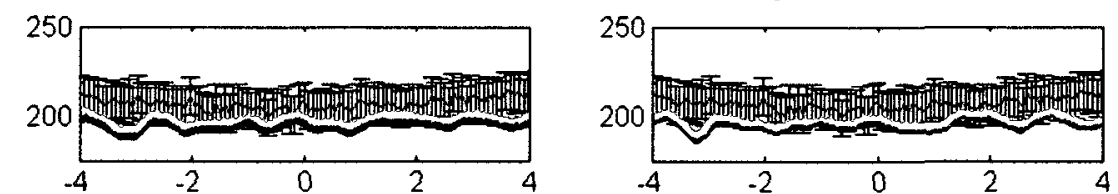

In the middle
of lung slab.
$2.0 \mathrm{~cm}$ from
phantom
surface

Directly below lung slab.

$3.0 \mathrm{~cm}$ from

phantom

surface

\begin{tabular}{|l|}
\hline $\mathrm{cm}$ below \\
lung slab. \\
$4.0 \mathrm{~cm}$ from \\
phantom \\
surface \\
\hline
\end{tabular}
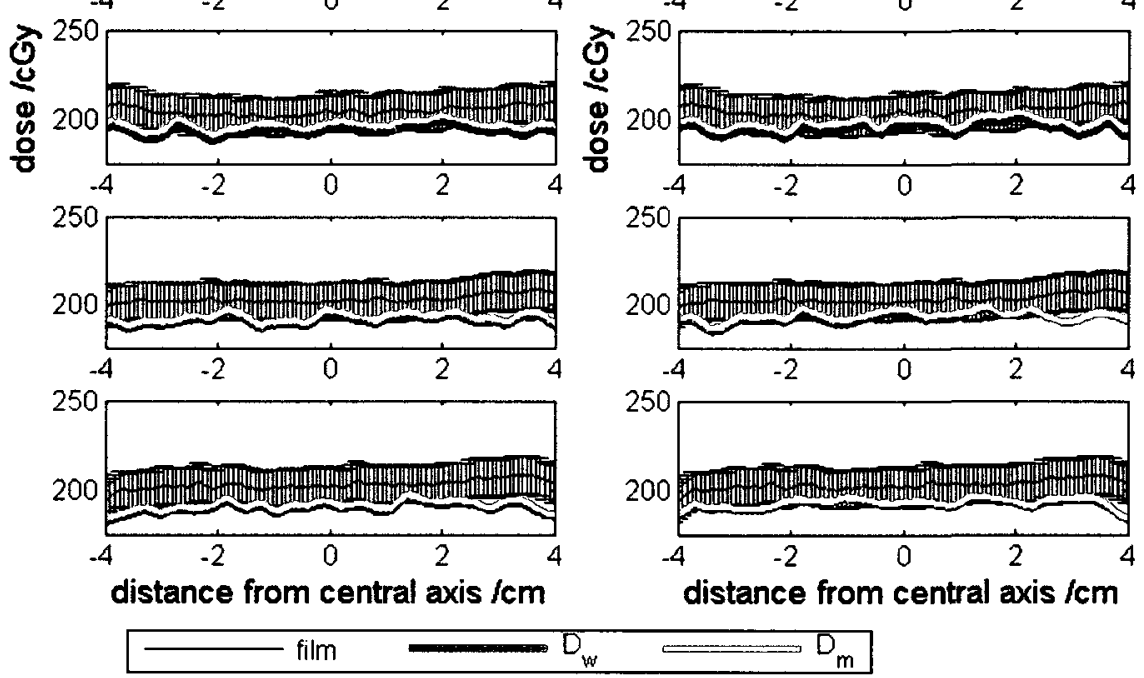

Figure 46. $17 \mathrm{MeV}$ film measurements and calculations at various depths within the lung slab phantom. To the left are the film measurements and vector based calculations, to the right are the film measurements and the image based calculations.

With exception to the profiles taken $1.0 \mathrm{~cm}$ from the phantom surface, the vector and image-based calculations lie within the measurement uncertainties. The $17 \mathrm{MeV}$ calculations agree better with the measured values than the $9 \mathrm{MeV}$ calculations.

Film was chosen as a tool for these experiments since unlike other dosimeters is can easily be placed between layers of inhomogeneities. However, it was found that the Gafchromic EBT1 film, as a tool for correlating film measurements with $\mathrm{D}_{\mathrm{w}}$ calculations, is not sufficiently precise. If the experiments were to be repeated, another approach would need to be taken. However, using the equipment and instruments available at the Ottawa General Hospital, we would be restricted from taking measurements above, between or immediately below an inhomogeneity. 


\section{Chapter 7}

\section{Retrospective Patient Data Analysis}

This study examined the dosimetric differences of the MC calculated electron beam plans when $D_{w}$ or $D_{m}$ is used in the dose evaluation for 54 breast and 15 head and neck cancer patients. The retrospective radiation treatment plans of breast, and head and neck cancer patients treated 2003-2008 with electron beams ranging from 6-20 MeV were retrieved from a patient database for review. We show that calculating treatment plans using the $D_{w}$ rather than the $D_{m}$ approach introduces differences in the DVHs of target and critical volumes. These plans were originally calculated using the $D_{m}$ method and were recalculated using the $D_{w}$ method. For comparison, the details of the original beam arrangement and the calculation parameters (number of histories and voxel size) were kept constant for both methods. Once calculated, the plans for $\mathrm{D}_{\mathrm{w}}$ and $\mathrm{D}_{\mathrm{m}}$ were compared quantitatively using the dose volume histograms and qualitatively by comparing the isodose distributions on the corresponding CT slices.

\subsection{Breast Cancer Patients}

Differences between $D_{m}$ and $D_{w}$ were observed in clinical breast patient plans, with magnitudes dependent not only on the beam energy but also on the location of the tumor and organs at risk, as previously suggested by Ding et $a l^{10}$. Figure 47 displays the dose volume histogram for $D_{w}$ and $D_{m}$ calculations for a treatment plan of the chest wall using a $13 \mathrm{MeV}$ electron beam at $115 \mathrm{~cm}$ SSD following a complete mastectomy. 


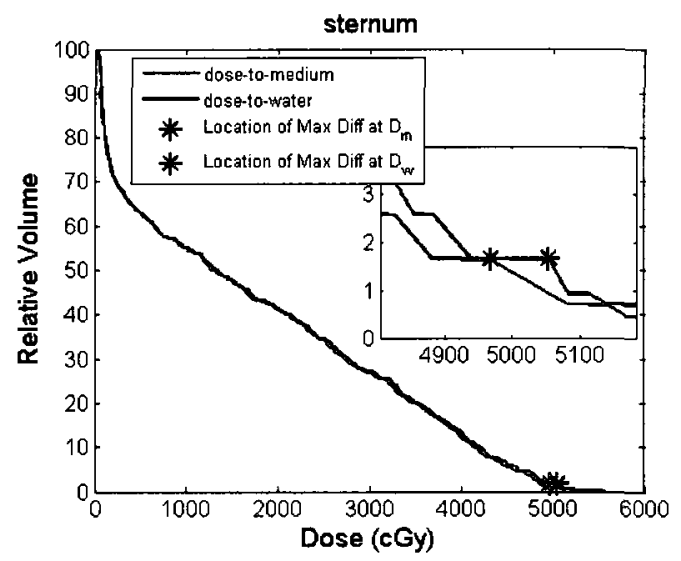

(a)

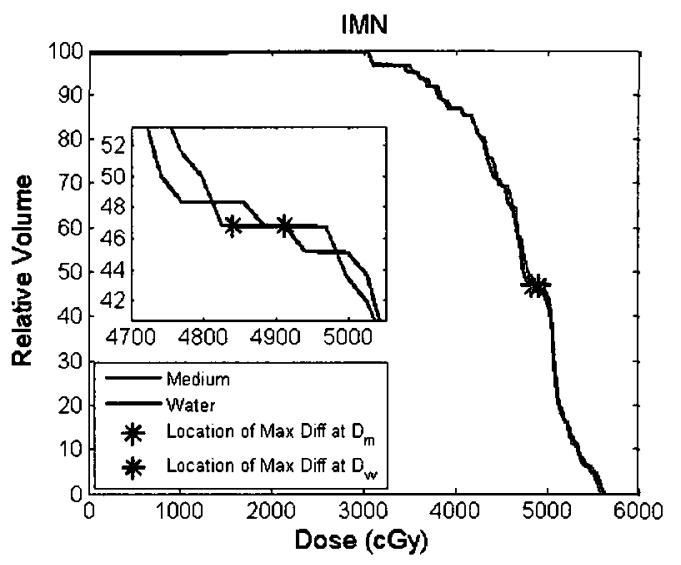

(b)

Figure 47. DVH comparison of $D_{w}$ and $D_{m}$ for a right breast mastectomy patient treated with a $13 \mathrm{MeV}$ electron beam, at $S S D=115 \mathrm{~cm}$, illustrating subtle dose differences in (a) the sternum and (b) the internal mammary nodes.

Figure 47 (a) shows the DVH of the sternum. The location of maximum difference between the two calculations is indicated by the asterix. At the point of maximum difference in the DVH the dose to the sternum was 49.9 Gy and $50.7 \mathrm{~Gy}$ for the $\mathrm{D}_{\mathrm{m}}$ and $\mathrm{D}_{\mathrm{w}}$ approach, respectively. This amounts to $1.6 \%$ difference in the maximum dose delivered to $1.6 \%$ of the volume. Figure 1 (b) is the DVH of the internal mammary nodes for the same patient. The dose received by the internal mammary nodes at the point of maximum difference in the DVH was 48.4 Gy and 49.1 Gy for $D_{m}$ and $D_{w}$ plans, respectively, to $46.8 \%$ of the volume. This corresponds to a $1.5 \%$ difference. 


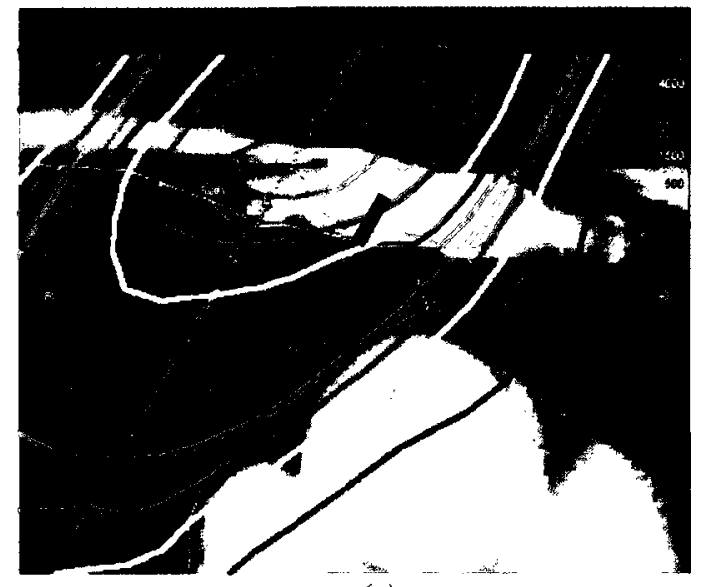

(a)

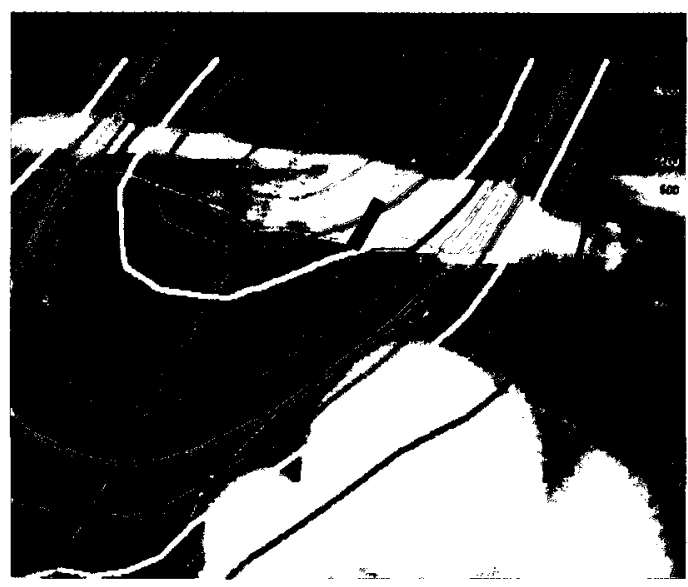

(b)

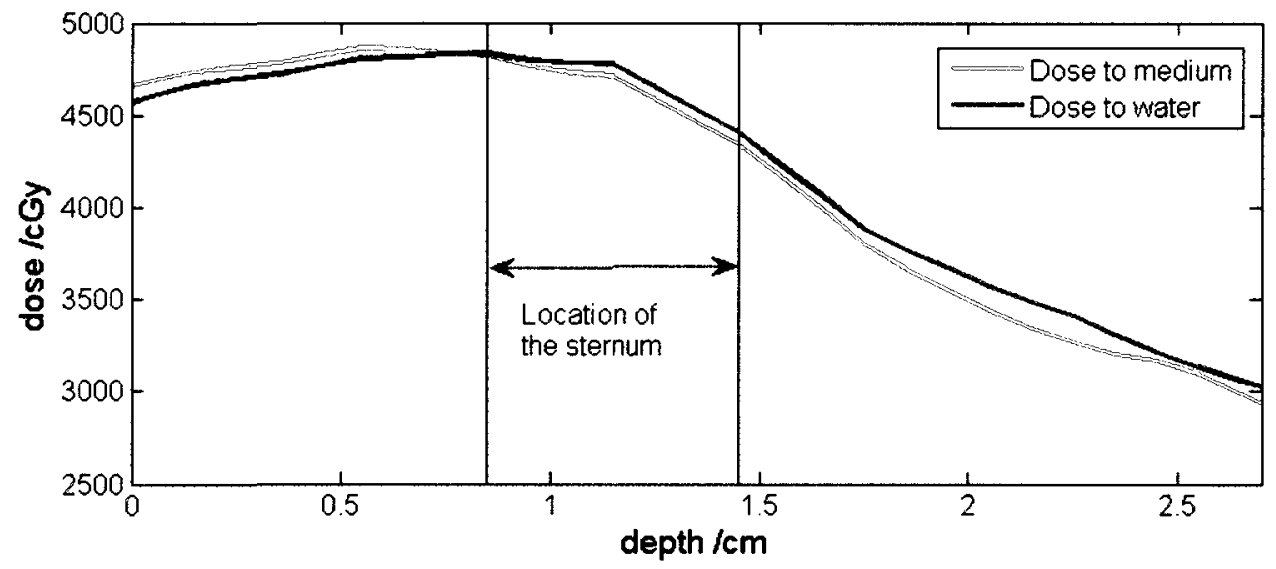

(c)

Figure 48. Isodose line distributions, calculated for $D_{w}$ (a) and $D_{m}$ (b) for a patient treated with $13 \mathrm{MeV}$ electrons. There is little visual difference between these plans. Blue dashed lines on (a) and (b) indicate the depth at which the lateral dose profiles in (c) were taken.

The $D_{m}$ and $D_{w}$ dose calculations on the central transverse patient slice are shown in Figure 48. As shown in Figure 48 (a) and (b), the isodose lines do not visibly differ between the two dose distributions. Figure 48 (c) is the profile taken through the transverse slice, indicated by the dashed blue line in Figure 48 (a) and (b). The profile was selected to pass through the sternum, since phantom studies have shown that larger differences exist in 
materials of higher electron densities. The largest difference found was $4.2 \%$ at a depth of $2.3 \mathrm{~cm}$ from the surface, within the lung.

Table 5 lists the mean $D_{w}$ to $D_{m}$ ratios of DVHs for the contoured GTVs, CTVs, surgical beds, lungs and hearts of the breast patients analyzed in this study. The ranges of the mean values are presented in square brackets. For breast patients, the plans calculated for $D_{m}$ and $D_{w}$ showed only very small differences. This is to be expected, as the irradiated tissues are not substantially different in composition from water. Larger differences are expected for the sites where hard bone is present in the field, such as head and neck patients.

Table 5 The mean and range of $D_{w}$-to- $D_{m}$ ratios in $D V H$ for the breast patients analyzed. The table is analogous to that presented by Dogan et al ${ }^{26}$.

\begin{tabular}{lcccc}
\hline $\begin{array}{c}\text { Volume } \\
\text { (number of } \\
\text { patients) }\end{array}$ & $\left(\frac{D_{w}^{90}-D_{m}^{90}}{D_{w}^{90}}\right) \times 100$ & $\left(\frac{D_{w}^{50}-D_{m}^{50}}{D_{w}^{50}}\right) \times 100$ & $\left(\frac{D_{w}^{2}-D_{m}^{2}}{D_{w}^{2}}\right) \times 100$ & $\left(\frac{D_{w}-D_{m}}{D_{w}}\right) \times 100$ \\
\hline GTV (23) & $-0.12[-1.96-2.24]$ & $-0.26[-1.85-1.91]$ & $-0.38[-2.62-1.85]$ & $-0.46[-3.14-5.26]$ \\
CTV (15) & $0.01[-0.02-0.13]$ & $0.00[-0.03-0.02]$ & $0.00[-0.02-0.02]$ & $-0.01[-0.04-0.03]$ \\
$\begin{array}{l}\text { Surgical Bed } \\
(10)\end{array}$ & $0.16[-1.12-2.20]$ & $0.17[-0.85-2.03]$ & $0.05[-1.38-1.55]$ & $-0.10[-2.58-2.31]$ \\
Lung (43) & $0.96[-3.48-11.82]$ & $2.10[-2.20-8.86]$ & $3.34[-2.04-8.04]$ & $1.99[-4.79-7.44]$ \\
Heart (21) & $0.80[-2.90-5.36]$ & $0.033[-8.91-4.07]$ & $0.95[0.01-8.04]$ & $0.18[-11.36-3.75]$ \\
\hline
\end{tabular}

\subsection{Head and Neck Patients}

The mean $D_{w}$ to $D_{m}$ ratios of DVHs for the contoured regions of the head and neck patients analyzed are similar to those in the breast cancer study. For the head and neck 
patients, the plans calculated for $D_{m}$ and $D_{w}$ showed only very small differences. This was to be expected, as the contoured tissues are not considerably different in composition from water. Larger differences were seen in the areas where hard bone was present, such as the skull.

These differences between $D_{m}$ and $D_{w}$ isodose distributions were quantified using dose profiles along the beam's axis. For the analysis of the dose profiles, the maximum percent difference between $D_{m}$ and $D_{w}$ was calculated with respect to the maximum dose along the profile for that patient.

We have examined dose profiles for several patients taken through cortical bone. Figure 49 displays the $D_{m}$ and $D_{w}$ profiles for a patient treated with a $6 \mathrm{MeV}$ beam positioned perpendicular to the right side of the forehead. Figure 49 (c) displays the dose profile through the patient's forehead, denoted by the dotted lines on the $D_{m}$ and $D_{w}$ isodose distributions in Figure 49 (a) and (b), respectively. Along the axis, the RED of the skull ranges from 1.68 and 1.72 .

The $D_{m}$ and $D_{w}$ dose calculations on the central transverse patient slice are shown in Figure 49 (a) and Figure 49 (b). As shown in the isodose distributions, there is a visible difference between the isodose lines of the two dose figures. The $45 \mathrm{~Gy}$ isodose line of the $\mathrm{D}_{\mathrm{w}}$ distribution encompasses the edge of the skull, upstream from the beam, while the $D_{m}$ distribution, this is not the case. Moreover, the $50 \mathrm{~Gy}$ isodose areas are noticeably different between the two distributions. 


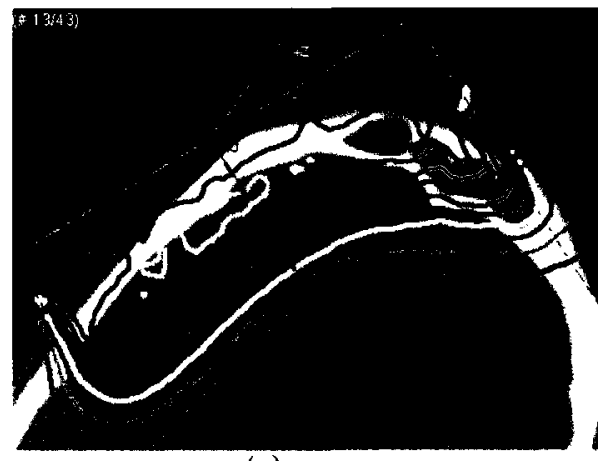

(a)

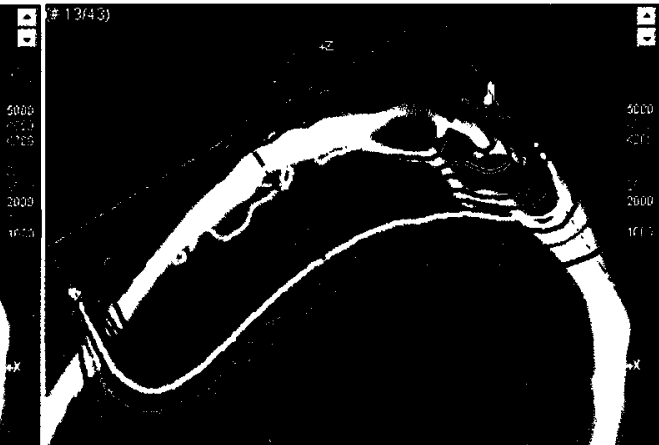

(b)

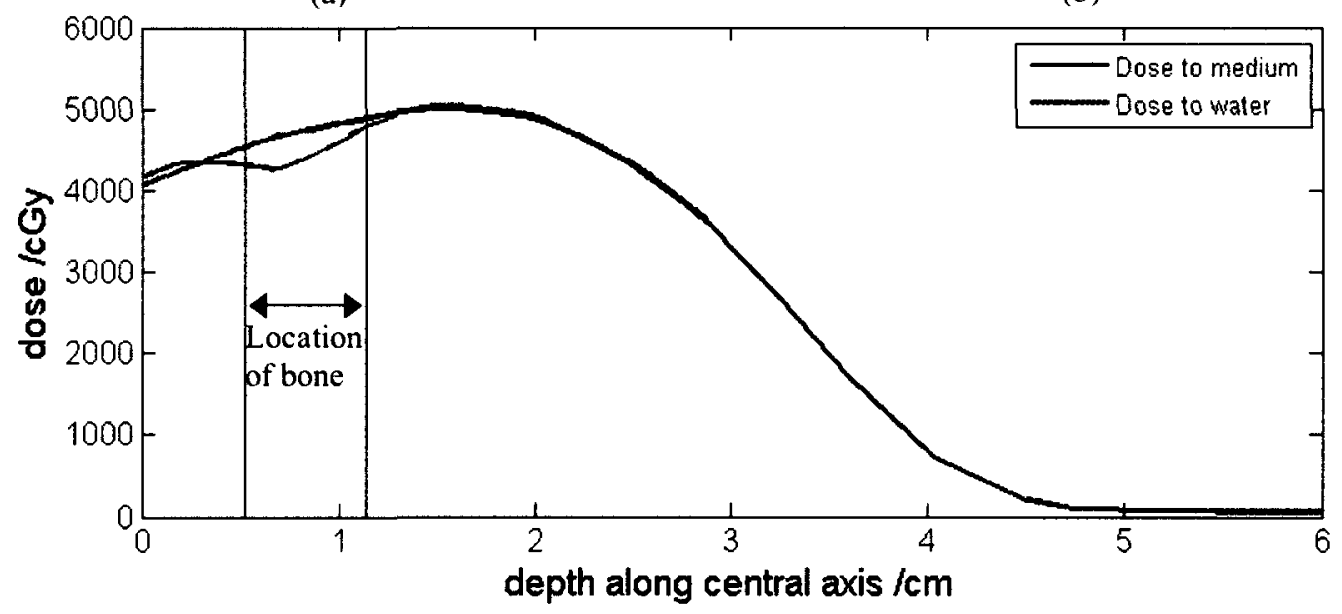

(c)

Figure 49. $D_{m}$ and $D_{w}$ profiles of a patient treated with a $6 \mathrm{MeV}$ beam positioned perpendicular to the patient's forehead on the right side. (a) $D_{m}$ isodose distribution. (b) $D_{w}$ isodose distribution. (c) $D_{m}$ and $D_{w}$ profiles through the forehead, denoted by the dotted lines in (a) and (b), respectively. There is a maximum difference of $7.9 \%$ between the $D_{m}$ and $D_{w}$ profiles, where $D_{w}$ exceeds $D_{m}$.

Figure 49 (c) are the profiles taken through the transverse slices, indicated by the dashed red line in Figure 49 (a) and Figure 49 (b). The maximum difference between the $D_{w}$ and the $\mathrm{D}_{\mathrm{m}}$ along the profile is $7.9 \%$ and is within the bone. The dose calculated in the bone is lower when using the $D_{m}$ method. This difference in the patient $D_{m}$ and $D_{w}$ profile is in close agreement with water-to-bone mass collisional stopping powers ratio, 1.098 . The mass collisional stopping power ratio was calculated using values obtained from 
NIST ESTAR ${ }^{18}$, evaluated at a depth of $0.24 \mathrm{~cm}$ within the bone, which corresponds to beam energy of $7.19 \mathrm{MeV}$.

For head and neck patients, dose to the bone was continually larger when calculating dose-to-water rather than dose-to-medium. Differences between plans depended on beam energy, as well as the location of the tumour and organs at risk, and are consistent with the water-to-bone mass collisional stopping power ratios. 


\section{Chapter 8}

\section{Conclusions}

For phantoms containing lung inhomogeneities, plans calculated for $D_{w}$ and $D_{m}$ showed only very small differences, less than $2 \%$. Larger differences were seen for phantoms where hard bone or air inhomogeneities were present in the field. In the case of the 9 $\mathrm{MeV}$ bone slab vector-based phantom we have seen differences as large as $12 \%$. Differences of these magnitudes were also present in the bone cylinders of the trachea and spine phantom. For the air cylinder phantoms the differences were roughly $5 \%$ for both the vector and image based phantoms. The extent of these differences depended on the material through which the electrons were propagated and the energy of the incident beam. Differences between the two calculation approaches were larger for phantoms containing inhomogeneities whose densities differed most from water, and for plans that used lower energy beams.

Some limitations existed when representing physical epoxy resin-based phantoms using vector-based anatomies. Image based-anatomies (such as the physical epoxy resin-based phantoms and patient anatomies) are much more complex than the vector-based phantoms depicted in Chapter 6.2. The vector-based phantoms had well defined structures and uniform electron densities. This is not the case for imaged-based anatomies. The boundaries of structures can become blurred to due to artefacts of the CT image or the CT image resolution, and the actual physical properties can vary within a structure. Not only can these add to the uncertainties of the calculations, but they may also create some discrepancies between the vector and image-based phantoms. 
The GafChromic EBT1 film was not sufficiently precise to be correlated to the $D_{w}$ calculations. The relative combined uncertainties $(\sim 10 \%)$ of the film for the range of doses we were interested in (150 to $225 \mathrm{cGy}$ ) were comparable to the largest differences seen between $D_{m}$ and $D_{w}$ calculations. These uncertainties were too large when trying to discriminate between calculations that differ by as little as $1.5 \%$.

For the breast cancer patients studied, the plans calculated for $D_{w}$ and $D_{m}$ showed only very small differences. This is to be expected, as the irradiated tissues are not substantially different in composition from water (the stopping power ratios for those tissues in respect to water are close to 1). Larger differences were seen for head and neck patients, where sites with hard bone are present in the field. For the head and neck patients analysed, dose to the bone was continually larger when calculating $D_{w}$ rather than $D_{m}$. However, for the other irradiated tissues, such as the brainstem, spinal cord and GTV, the differences between the calculated plans were small. The differences observed in soft tissues for the breast, and head and neck patients were all within the recommended accuracy $^{39}$ of $5 \%$ for clinical dose calculations. Differences between patient plans calculated with $D_{m}$ and $D_{w}$ depended on beam energy, as well as the location of the tumor and organs at risk, and are consistent with the water-to-tissue stopping power ratios, and in agreement with Ding et $a l^{10}$.

Monte Carlo is the most accurate method of calculating dose since it is based on the direct simulation of radiation transport. It has the ability to calculate $\mathrm{D}_{\mathrm{m}}$ or $\mathrm{D}_{\mathrm{w}}$. For the clinical cases studied here, the differences between the $D_{m}$ and $D_{w}$ calculations are minor. 
Nonetheless, calculating patient plans using the $D_{w}$ method defeats one of the major benefits of using a MC-based treatment planning system. Based on the recommendations of an AAPM task group ${ }^{4}$ and to remain consistent both options should be available in commercial software until a consensus is reached about which method is more suitable for clinical dose calculations and evaluation of treatment outcomes. 


\section{REFERENCES}

${ }^{1}$ E. J. Hall, Radiobiology for the Radiologist. (J. B. Lippincott Company, 1994, Philadelphia), p. 2.

${ }^{2}$ E.B. Podgoršak, Radiation physics for medical physicists, (Springer, 2006, New York), p. 13.

3 J.E. Cygler, C. Lochrin, G.M. Daskalov, M. Howard, R. Zohr1, B. Esche1, L. Eapen, L. Grimard, J.M. Caudrelier, "Clinical use of a commercial Monte Carlo treatment planning system for electron beams," Med. Phys. 50, 1029-1034 (2005).

${ }^{4}$ P.R. Almond, P.J. Biggs, B.M. Coursey, W. F. Hanson, M. Saiful Huq, Ravinder Nath, D. W. O. Rogers, "AAPM's TG-51 protocol for clinical reference dosimetry of highenergy photon and electron beams” Med. Phys. 26, 1847-1870 (1999).

${ }^{5}$ M.S. Huq, P. Andreo, H. Song, "Comparison of the IAEA TRS-398 and AAPM TG-51 absorbed dose to water protocols in the dosimetry of high-energy photon and electron beams," Phys. Med. Biol. 46, 2985-3006 (2001).

${ }^{6}$ E. Gil, E. Vandervoort, B. Clark, JE Cygler, "Impact of Dm Vs. Dw in Electron Beam Monte Carlo Based Treatment Planning for Breast," Med. Phys. 36, 2617 (2009).

${ }^{7}$ E. Gil, B. Clark, J.E. Cygler, "Evaluation of Dosimetric Differences between Dose-toWater and Dose-to-Medium for Head and Neck Patients Treated with Electron Beams," Med. Phys. 36, 4318 (2009).

${ }^{8}$ H.H. Liu, P. Keall, " $D_{m}$ rather the $D_{w}$ should be used in Monte Carlo treatment planning," Med Phys 29, 922-924 (2002).

${ }^{9}$ I.J. Chetty, B. Curran, J. Cygler, et al., "Report of the AAPM Task Group No. 105: Issues associated with clinical implementation of Monte Carlo-based photon and electron external beam treatment planning A software tool for the quantitative evaluation of 3D dose calculation algorithms," Med. Phys. 34, 4818-53 (2007).

${ }^{10}$ G.X. Ding, D.M. Duggan, C.W. Coffey, P. Shokrani, J.E. Cygler, "First macro Monte Carlo based commercial dose calculation module for electron beam treatment planningnew issues for clinical consideration," Phys. Med. Biol. 51, 2781-2799 (2006).

11 D. Schulz-Ertner, O. Jäkel, W. Schlegel, "Radiation therapy with charged particles," Semin. Radiat. Oncol. 16 249-59 (2006). 
${ }^{12}$ ICRU 2004 Prescribing, Recording, and Reporting Electron Beam Therapy ICRU Report 71 (Bethesda, MD: International Commission on Radiation Units and Measurements).

${ }^{13}$ A. Brahme, H. Svensson, "Specification of electron beam quality from the central-axis depth absorbed dose distribution," Med. Phys. 3, 95-102 (1976).

${ }^{14}$ L. Katz, A.S. Penhold, "Range-energy relations for electrons and the determination of beta-ray endpoint energies by absorption," Rev. Mod. Phys. 24, 28-44 (1952).

${ }^{15}$ D. Harder, "Energiesspektren Schneller Elektronen in Verschiedenen Tiefen," In Montreux: Symposium on High-Energy Electrons (Berlin: Springer Verlag), (1965), p. 260.

${ }^{16}$ P. Mayles, A. E. Nahum, J. C. Rosenwald, Handbook of Radiotherapy Physics: Theory and Practice. (Taylor \& Francis Group, 2007, New York), p. 565.

${ }^{17}$ F. H. Attix, Introduction to Radiological Physics and Radiation Dosimetry. (John Wiley \& Sons, 1986, Federal Republic of Germany), p. 184.

${ }^{18}$ NIST http://physics.nist.gov/PhysRefData/Star/Text/ESTAR.html.

${ }^{19}$ B. B. Rossi, High Energy Particles (Prentice Hall, New York, 1952).

${ }^{20}$ F.M. Khan, The Physics of Radiation Therapy. (Williams \& Wilkins, 1984, Baltimore), p. 288.

${ }^{21}$ I. Kawrakow, M. Fippel, K. Friedrich, "3D electron dose calculations using a voxel based Monte Carlo algorithm (VMC)," Med. Phys. 23, 445-457 (1996).

${ }^{22}$ Kawrakow I, "Improved modeling of scattering in the Voxel Monte Carlo model," Med. Phys. 24, 505-517 (1997).

${ }^{23}$ M. Fippel, I. Kawrakow, K. Friedrich K. "Electron beam dose calculations with the VMC algorithm and the verification data of the NCI working group," Phys. Med. Biol. 42, 501-520 (1997).

${ }^{24}$ MDS Nordion (2002) DCM Algorithms White Paper, Document ID: DC-020-MPUGB_DCM-ALGv1.

25 J.E. Cygler, G.M. Daskalov, G.H. Chan, G,X. Ding, "Evaluation of the first commercial Monte Carlo dose calculation engine for electron beam treatment planning," Med. Phys. 31, 142-153 (2004). 
${ }^{26}$ N. Dogan, J.V. Siebers, P.J. Keall, "Clinical comparison of head and neck and prostate IMRT plans using absorbed dose to medium and absorbed dose to water," Med. Phys. 51, 4967-4980 (2006).

${ }^{27}$ J.V. Siebers, P.J. Keall, A.E. Nahum, R Mohan, "Converting absorbed dose to medium to absorbed dose to water for Monte Carlo based photon beam dose calculations," Phys. Med. Biol. 45, 983-95 (2000).

${ }^{28}$ J.K. Gardner, J.V. Siebers and I. Kawrakow, "Comparison of two methods to compute the absorbed dose to water for photon beams," Med. Phys. 52, N439-N447 (2007).

${ }^{29}$ D.R. White, R.J. Martin, R. Darlison, "Epoxy resin based tissue substitutes," Br. J. Radiol. 50, 814-821 (1977).

${ }^{30}$ D. R. White, C. Constantinou, J. Martin, "Technical note: Foamed Epoxy resin-based lung substitutes," Br. J. Radiol. 59, 187-790 (1986).

31 G.X. Ding, J.E. Cygler, G.G. Zhang, M.K. Yu, "Evaluation of a commercial threedimensional electron beam treatment planning system," Med. Phys. 26, 2571-2580 (1999).

${ }^{32}$ J. Cygler, J.J. Battista, J.W. Scrimger, E. Mah, J. Antolak, "Electron dose distributions in experimental phantoms: a comparison with 2D pencil beam calculations," Med. Phys. 32, 1073-1086 (1987).

${ }^{33}$ G.X. Ding, J.E. Cygler, C.W. Yu, N.I. Kalach, G. Daskalov, “A comparison of electron beam dose calculation accuracy between treatment planning systems using either a pencil beam or a Monte Carlo algorithm," Int. J. Radiat. Oncol. Biol. Phys. 23, 622-633 (2005).

${ }^{34}$ C.M. Wells, T.R. Mackie, M.B. Podgorsak et al. "Measurements of the electron dose distribution near inhomogeneities using a plastic scintillation detector," Int. J. Radiat. Oncol. Biol. Phys. 29, 1157-1165 (1994).

${ }^{35}$ F.C. Su, Y. Liu, S. Stathakis, C. Esquivel, N. Papanikolaou, "Dosimetry characteristics of GAFCHROMIC EBT film responding to therapeutic electron beams," Appl. Radiat. Isot. 65, 1187-1192 (2007).

${ }^{36}$ M.J. Butson, T. Cheung, P.K. Yu, "Scanning orientation effects on Gafchromic EBT film dosimetry," Australas. Phys. Eng. Sci. Med. 29, 281-284 (2006).

${ }^{37}$ International Specialty Products (2005) GAFCHROMIC® EBT: Self-Developing film for radiotherapy dosimetry White Paper, Wayne, New Jersey. 
${ }^{38}$ S. Devic, J. Seuntjens, G. Hegyi, E.B. Podgorsak, C.G. Soares, A.S. Kirov, I. Ali, J.F. Williamson, A. Elizondo, "Dosimetric properties of improved GafChromic films for seven different digitizers," Med. Phys. 31, 2392-2401 (2004).

${ }^{39}$ N. Papanikolaou, J.J. Battista, A.L. Boyer, et al., "AAPM Report of Task Group Report No. 65 of the Radiation Therapy Committee of the American association of Physicists in Medicine 2004, Tissue inhomogeneity corrections for megavoltage photon beams AAPM Report No. 85, 1-135. 